
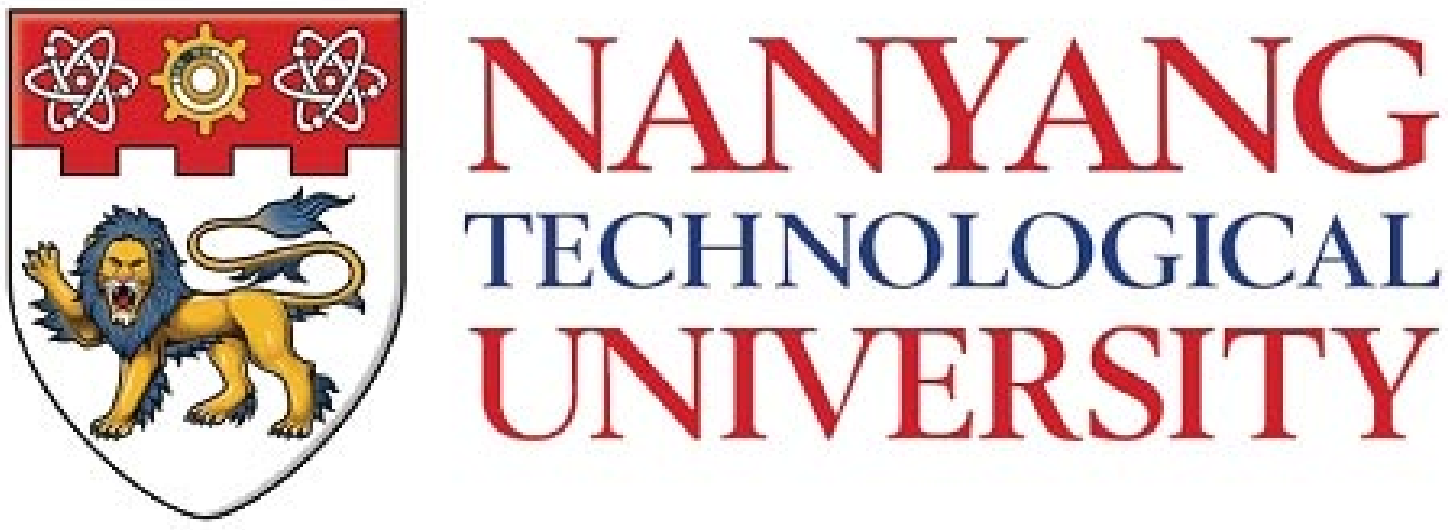

\title{
Responsive supramolecular prodrug nanoparticles for cancer therapy
}

\author{
Chen Hongzhong
}

School of Physical and Mathematical Sciences

A thesis submitted to the Nanyang Technological University in partial fulfilment of the requirement for the degree of Doctor of Philosophy 


\title{
Responsive supramolecular prodrug nanoparticles for cancer therapy
}

\author{
Chen Hongzhong
}

School of Physical and Mathematical Sciences

A thesis submitted to the Nanyang Technological University in partial fulfilment of the requirement for the degree of Doctor of Philosophy 


\section{Acknowledgments}

I would like to thank my supervisor, Associate Professor Zhao Yanli for his enduring patience and faith in me throughout my PhD years. Prof. Zhao has graciously granted me the freedom to explore my own research interests over these 4 years, which allowed me to reach my full potential.

I would also like to extend my gratitude to Assistant Professor Chen Gang, for opening up collaboration opportunities for me, enabling me to expand my horizons and gather new insights from a wider network of researchers. Credit also goes to my collaborator from the SBS who has also enriched my research experience.

I would also like to thank all my group mates, especially Miss Phoebe Tham, for their valuable advice, suggestions and tremendous support.

The Division of Chemistry and Biological Chemistry, School of Physical and Mathematical Sciences of Nanyang Technological University has also provided me with state-of-the-art research facilities, which contributed immensely to the progress of my research.

Last but not least, the unwavering support from my family and friends has allowed me to pursue my interest without any worries.

These four years may have been challenging with countless of setbacks. Through it all, I have learnt to persevere, go headstrong, and work smart. Above all, I have also learnt the meaning of teamwork and collaboration in the research industry as no man is an island. 
As I close this chapter of my life, my greatest appreciation goes out to the abovementioned people. Without the help of these people, I would not have made it this far, and I will not be who I am today, for which I am deeply grateful for. 


\section{Contents}

Acknowledgments

Chapter 1 Background and introduction

1.1 Drug delivery system and cancer therapy

1.2 Prodrug and prodrug self-assembly for cancer therapy

1.2.1 Reduction environment stimuli responsive prodrugs

1.2.2 ROS or hydrogen peroxide triggered prodrug.

1.2.3. $\mathrm{pH}$ responsive prodrug.

1.2.4 UV triggered prodrug

1.2.5 NIR and two photon triggered prodrug.

1.2.6 Enzyme triggered prodrug

1.2.7 Hypoxia triggered prodrug 35

1.3 Supramolecular chemistry and its biomedical application 38

1.3.1 Hydrogels or supramolecular hydrogels for drug delivery 39

1.3.2 Liposome and polymersome for drug delivery 42

1.3.3 Host-Guest chemistry and applications in biomedical 45

Chapter 2 Prodrug Self-assembled Glutathione-responsive Vesicle for Targeted Chemotherapy in Combination with Intracellular Imaging 59

2.1 Introduction 59 
2.2.1 Synthesis of the compounds

2.2.2 Preparation of vesicles. 67

2.2.3 Drug release mechanism 68

2.2.4 Cellular uptake study 68

2.2.5 Cytotoxicity study 69

2.3 Results and discussion 69

2.3.1 Design and synthesis of the amphiphilic small molecule prodrug 69

2.3.2 Self-assembly of the amphiphilic small molecule prodrug. 70

2.3.3 GSH triggered drug release behavior 73

2.3.4 Cellular uptake study 76

2.3.5 In vitro antitumor study. 81

2.4 Conclusion 82

Chapter 3: Dendritic Supramolecular Theranostic Prodrug Vesicle Delivery of siRNA for Responsive Imaging Guided Synergetic Cancer Therapy. 84

3.1 Introduction 84

3.2 Materials and Characterizations 88

3.2.1 Synthesis of the compounds. 90

3.2.3 Preparation of vesicles and siRNA loading vesicles. 94 
3.2.5 Drug release behavior

3.2.6 Cellular uptake study .95

3.2.7 In vitro cytotoxicity study 96

3.3 Results and discussion 96

3.3.1 Design and synthesis of the supramolecular amphiphiles prodrug 96

3.3.2 Self-assembly behaviors of the supramolecular amphiphiles prodrug 97

3.3.3 GSH responsive behaviors 100

3.3.4 Cellular uptake study 105

3.3.5 Cytotoxicity and in vitro antitumor study 106

3.3.6 SiRNA delivery study 107

3.4 Conclusion

Chapter 4: Bio-degradable Bola Type Supramolecular AIE Vesicle for delivery of siRNA

4.1 Introduction

4.2 Materials and Characterizations

4.2.1 Synthesis of the compounds

4.2.3 Preparation of vesicles and siRNA loading vesicles. 123

4.2.4 Gel retardation assay and siRNA/vesicles complex protection assay 124 
4.2.5 Cellular uptake study

4.2.6 In vitro cytotoxicity study 125

4.3 Results and discussion 125

4.3.1 Design and synthesis of the bola type supramolecular AIE vesicle 125

4.3.2 Study of the AIE properties of the guest molecule G1

4.3.3 Self-assembly behaviors of the supramolecular amphiphiles bola type AIE vesicle.

4.4 Conclusion

Conclusion and outlook

References 140

List of the publications 148 


\section{Abstract}

Prodrugs are modified drugs which can be converted to the original form after uptake by tumor or activation by external stimuli. Advantages of prodrugs include better water solubility, higher selectivity, longer circulation time, and controlled release than the free anticancer drugs. Common prodrugs still have an obvious drawback that is low drug content due to inherent balance between the hydrophobic part with the hydrophilic part. Therefore, construction of prodrugs using small molecules with high drug loading percentage and inherent amphiphilic property has attracted a lot of attention. To obtain better therapeutic performance, a lot of external stimuli strategies have been introduced to achieve on-demand drug release during the design of prodrugs. Benefiting from the host-guest chemistry, supramolecular amphiphiles show numerous advantages, because fabrication was achieved by the means of various non-covalent interactions. Supramolecular amphiphilies are constructed by two parts with various noncovalent interactions, which efficiently simplify the tedious covalent synthesis and functional modification of the traditional amphiphiles. Owing to the advantages mentioned above, supramolecular amphiphiles have garnered tremendous interest of the researchers, especially in biomedical relevant fields. Gene therapy has gained considerable attention in the past decades, due to its promising therapeutic application in genetic diseases, cancer, cardiovascular and infectious diseases. However, due to its rapid degradation by serum nucleases in the blood, there are still on-going efforts to develop effective carrier vectors. 
As mentioned above, my research mainly focuses on the design, synthesis and characterization of small molecular prodrugs and the preparation of supramolecular amphiphilic nanosize aggregates by using prodrugs and macrocycles for cancer therapy. Furthermore, development of safe and efficient vesicles to deliver the siRNA for cancer therapy is another goal in my $\mathrm{PhD}$ research.

Therefore, in my $\mathrm{PhD}$ research, I developed methods to fabricate supramolecular amphiphilic prodrug nano-aggregates and extended the usage to deliver siRNA for cancer therapy. 


\section{Chapter 1 Background and introduction}

\subsection{Drug delivery system and cancer therapy}

Development of new improved treatment for diseases is the eternal pursue of mankind. Interest in discovering of novel and improved treatment for cancer remains. Cancer is a group of diseases characterized by abnormal growth of body cells which spread to other tissues (cancer metastasis) ${ }^{7,8}$. Because of metastasis, treatment of cancer was difficult, resulting in high mortality rate. Many treatment techniques are available for cancer therapy ${ }^{9}$. The commonly used treatments include surgery, chemotherapy, photodynamic/thermal therapy, radiation therapy, and immunotherapy ${ }^{10-12}$. Advances in chemical medicine provide a powerful weapon to cure diseases, but side effects of chemotherapy remains inevitable. Large amount of efforts have been made to relieve the side effects of the chemotherapy by the development of drug delivery systems (DDS) which inherently offer several advantages such as targeted drug delivery, reduced dosage of drug used, as well as the improved internalization and accuracy in tumour site uptake. DDS are sophisticatedly designed to provide efficient chemotherapy with minimal side effects. These systems include liposomes ${ }^{1}$, micelles $^{2}$, vesicles ${ }^{3}, \mathrm{MSNPs}^{4}$, and inorganic particles ${ }^{5}$, which were fabricated to improve the solubility of hydrophobic therapeutic agents, avoid degradation of the drug, enhance the selective uptake in lesions as well as offering external stimuli release properties. In the past decades, many evidences have shown the effectiveness of these systems application in chemotherapy. ${ }^{6}$ 

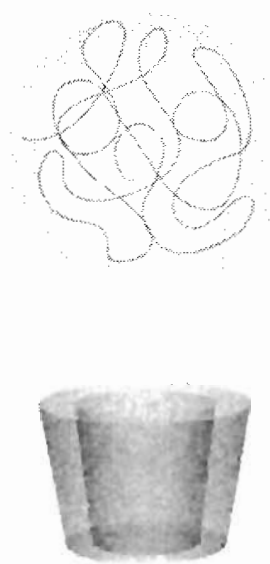

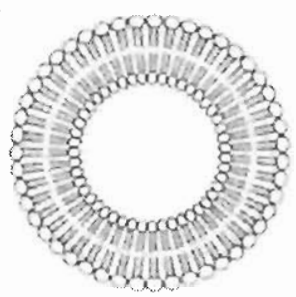

$\mathbf{f}$

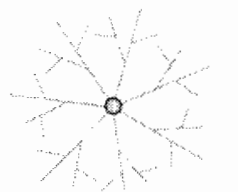

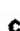

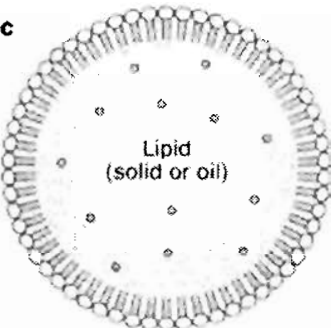

g

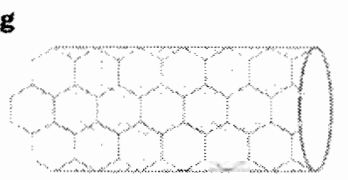

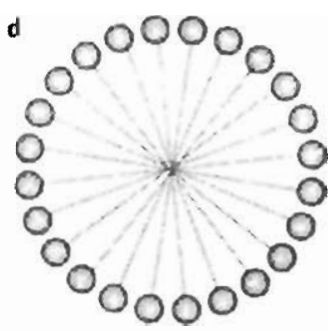

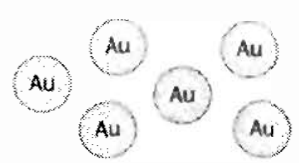

Figure 1 a. Polymeric nanoparticles; b. Liposomes; c. Lipid nanoparticles and nanoemulsions; $d$. Micelles,

e. Cyclodextrins; f. Dendrimers; $g$. Carbon nanotubes; h. Inorganic nanoparticles such as gold nanoparticles. (Reproduced with permission from ref 6. (Reproduced with permission from ref 6 .

Copyright 2015 Nature Publishing Group.)

Enhanced permeability and retention (EPR) effect is a kind of special property of solid tumor tissue. Because of the urgent need of nutrients to satisfy rapid growth, tumors develop a strategy to form a large number of blood vessels. These newly formed vessels are different from normal vessels in structure and property. This special property lead to the tumor's lack of effective lymphatic drainage and abnormal fluid transport dynamics, especially for nanosize particles with the diameters below $200 \mathrm{~nm}^{13-18}$

In order to utilize EPR effect, nano-sized carriers can be designed to improve the selective uptake by tumor sites, also known as the passive targeting strategy. For cancer chemotherapy or photodynamiclthermal therapy, DDS can efficiently enhance the selectivity uptake and extend circulation time to achieve better therapeutic performance as compared to original therapeutic agents ${ }^{19-22}$. 
In light of the benefits of nanotechnology, various nanoparticles have been widely used as drug carriers to improve the efficacy of the chemotherapy. There are two methodologies to deliver therapeutic agents using nanocarriers. One is the loading free drugs inside of these drug carriers by hydrophobic interaction or electrostatic absorption and another is the modification of drug molecules onto carrier's surface by covalent bonds. Both methods face several drawbacks, such as low drug loading percentage, minimal renal clearance, premature drug leakage and insufficient drug release $\mathrm{e}^{21,23-26}$.

\subsection{Prodrug and prodrug self-assembly for cancer therapy}

Prodrugs are modified drugs which can be converted to the original form after uptake by tumor or activation by external stimuli. Prodrugs offer better water solubility, higher selectivity, longer circulation time, and better controlled release as compared to free anticancer drugs, thus overcoming the shortfalls of most anticancer drugs. Similar to the other drug carriers, prodrugs could be fabricated into nanoparticles via self-assembly and thus harnessing the EPR effect during internalization. The most investigated prodrugs are in a form of polymer grafted drugs, which have been developed for decades and already have commercially available products which display great application potential in clinical treatment.

Polymeric prodrugs have an obvious drawback which is its low drug content in order to achieve a balance between the hydrophobic and hydrophilic component. Thus, the construction of prodrugs using small molecules with high drug loading percentage and inherent amphiphilic property has attracted tremendous attention. ${ }^{15,27-30}$ 
Another issue that need to be addressed is the controlled drug release of such prodrug. In account of the inherent strong covalent linkages used during fabrication of prodrug, it is difficult to control the release progress, resulting in the drugs released over too long a duration, decreasing specificity and efficacy. To achieve better therapeutic outcome, a lot of external stimuli strategies have been introduced to achieved on command drug release during design prodrugs ${ }^{20,24,31-36}$.

\subsubsection{Reduction environment stimuii responsive prodrugs}

GSH concentration is different in intracellular $(\sim 2-10 \mathrm{mM})$ and extracellular $(\sim 2-$ $10 \mu \mathrm{M})$ compartments. Interestingly, intracellular concentration of GSH in cancer cells is much higher than in normal cells. Disulfide bond is relatively stable in physiological conditions, but it can be cleaved after internalization by cancer cells. Disulfide bonds are widely introduced into the prodrugs design. Due to different GSH concentration in normal cells and cancer cells, prodrugs linked via disulfide bonds display rapid response to thiols and hence is able to achieve high selectivity. ${ }^{18,25,31,37-43}$

Recently, several research groups reported prodrugs based on GSH activated to release active form strategy. ${ }^{37,38,44,45}$ As shown in Figure 2, Kim et. al reported camptothecin (CPT) prodrug responsive to GSH. They designed a multicomponent prodrug comprised of four parts: a cyclic peptides containing RGD (Arg-Gly-Asp) sequence serving as targeting ligand, a naphthalimide unit serving as the fluorescent reporter, a disulfide bond serving as cleavage linker, and a CPT unit as a therapeutic ingredient. After uptake via $\alpha_{v} \beta_{3}$ integrin-mediated endocytosis, the disulfide bond was cleaved upon reaction with thiols in cancer cells followed by intramolecular 
cyclization to release the free CPT molecules and generate a new fluorescence emission at $535 \mathrm{~nm}$, compared with the original fluorescence at $480 \mathrm{~nm}$. The fluorescence changes were suitable to be used to monitor drug release process. This prodrug with inherent theranostic property show high selectivity tumor targeting and allows the monitoring of the drug concentration in real time, which allows real time dose control of drug and obtain better therapeutic performance ${ }^{46}$.

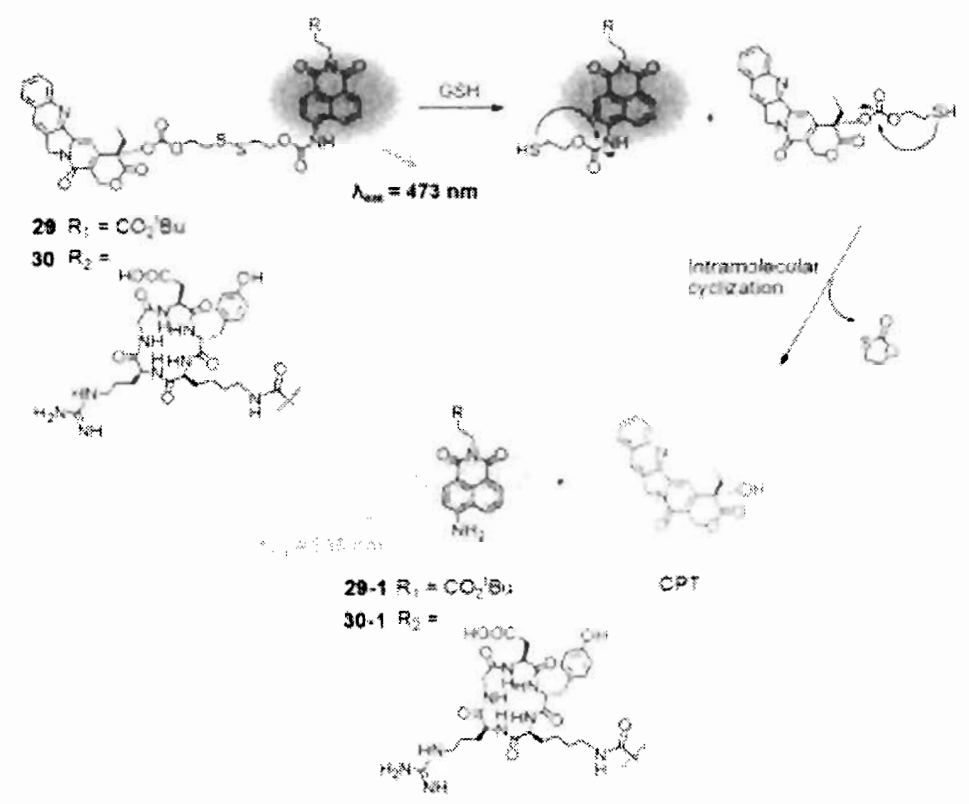

Figure 2. Prodrugs structure and proposed release mechanism. (Reproduced with permission from ref 46. Copyright 2013 American Chemical Society.)

For traditional polymeric micelles, there are several obvious drawbacks, including low drug loading percentage, low loading efficacy, and burst drug release. To resolve these important issues, Chen's group developed a dimer prodrug strategy, as shown in Figure 3. A prodrug comprising of two CPT molecules was prepared with a disulfide linker as the active site. Using the common co-precipitation method, the mixture of dimer prodrug and mPEG-PLA with a ratio of 1:1 (w/w) dissolved in DMF was 
precipitated in water to give uniform small aggregates with narrow size distribution about sub-200 $\mathrm{nm}$. In contrast, the mixture of free CPT and mPEG-PLA was precipitated in water at the same ratio forming particles at the micrometer scale with a relatively wide distribution. Negligible CPT release was observed in the case of the dimer CPT prodrug nanoparticles in absence of dithiothreitol (DTT), while sustainable release of CPT over $48 \mathrm{~h}$ was detected when DTT was added. In the same conditions, free CPT particles faced serious burst release, no matter presence or absence of DTT. This dimer strategy formed prodrug system displayed high loading about $50 \%$ with near to $100 \%$ loading efficacy and shown great prospects in construction drug delivery system ${ }^{47}$.
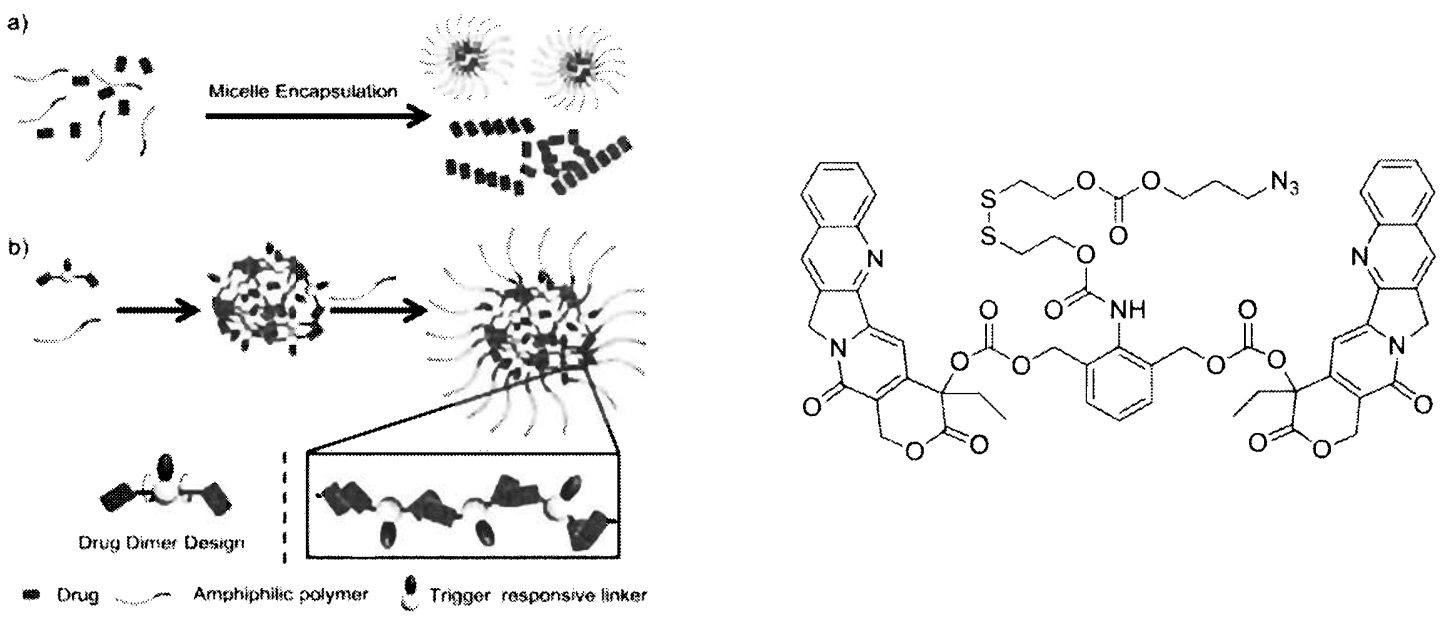

Figure 3 Hydrophobic drug encapsulated in polymeric micelles, prodrug nano-aggregates formation.

following with surface coating, and structure of the prodrugs. (Reproduced with permission from ref 47.

Copyright 2015 American Chemical Society.)

Liu and his team reported a polyprodrug amphiphiles, using CPT afford disulfide bond prodrug as hydrophobic part for shape regulated cellular internalization and drug delivery. As shown in Figure 4, CPT was modified with disulfide bond as monomer, 
followed by reaction with PEG-based RAFT agent to afford an amphiphilic polymeric prodrug. Using different preparation methodology, four types of nanoparticles were obtained with different size and morphology, spheres, smooth disks, large compound vesicles, and staggered lamellae. After endocytosis by cancer cells, disulfide bond was cleaved by GSH followed by intramolecular cyclization to release active CPT. In vitro experiments displayed that the staggered lamellae nanostructures showed the fastest cellular internalization rates, large composed vesicles nanostructures showed moderation release rates, but sphere nanostructures showed the lowest release rates ${ }^{48}$

a

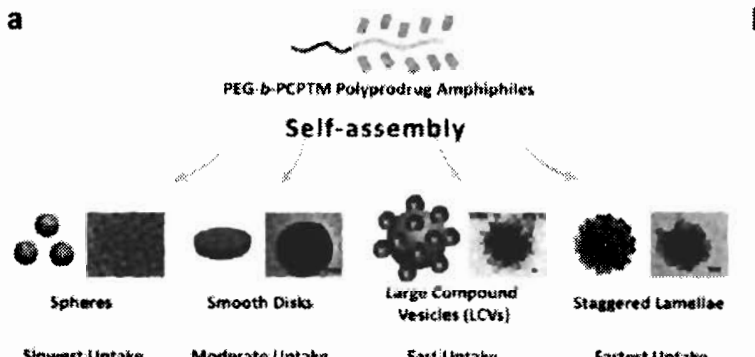

b
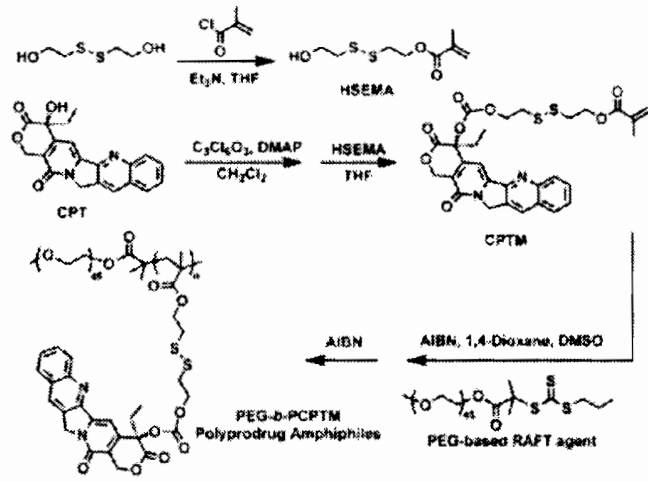

cmm

c
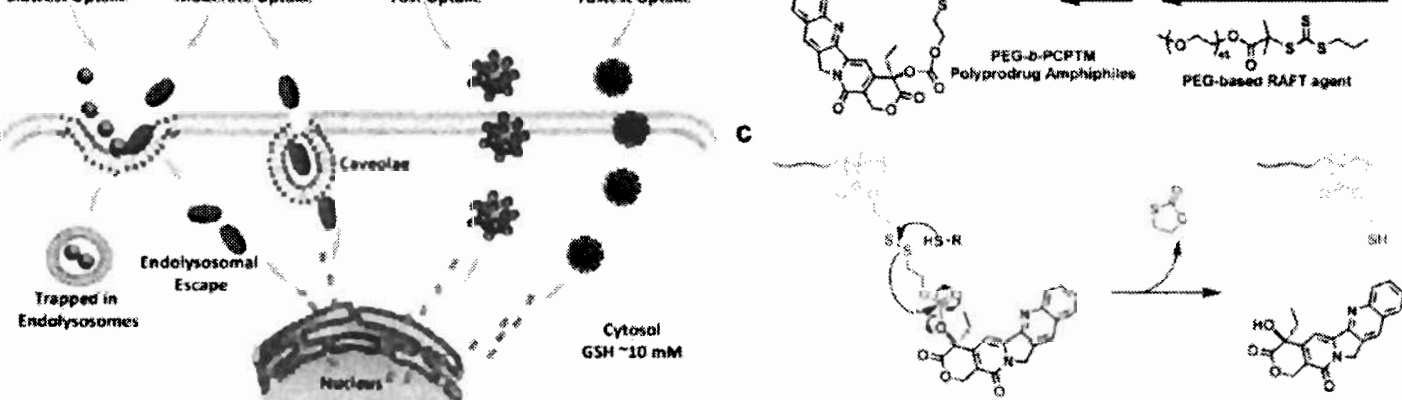

Figure 4 Schematic itlustration of four types nanostructures and synthetic routes of the polymeric amphiphilic prodrug. Reproduced with permission from ref 48. Copyright 2013 American Chemical Society.) 


\subsubsection{ROS or hydrogen peroxide triggered prodrug.}

Reactive oxygen species (ROS) are a group small molecules with high reactivity, including superoxide anions $\left(\mathrm{O}^{2-}\right),{ }^{49}$ hydrogen peroxide $\left(\mathrm{H}_{2} \mathrm{O}_{2}\right)^{19,50}$ and hydroxyl radical. ${ }^{51}$ ROS play an important role on regulation of redox balance within cells. It is known that cancer cells experienced raised intrinsic oxidative stress via accumulating ROS because of disrupted ROS homeostasis. In light of the higher ROS concentration in cancer cells, numerous prodrugs have been constructed based on $\mathrm{H}_{2} \mathrm{O}_{2}$ responsive release. The prodrugs based on $\mathrm{H}_{2} \mathrm{O}_{2}$ responsive mechanism should be composed of two functional components: a hydrogen peroxide activated moiety and an effective therapeutics ${ }^{24,49,51-53}$. One of the popular trigger is derivative from boronic acids or esters $^{54-58}$.

In the past decades, a lot of progresses have been achieved by using boronic acids or esters. Kim group reported a theranostic platform which was made up of four components. Biotin serves as a targeting ligand, which guides the theranostic platform to tumor sites. 5'-deoxy-5-fluorouridine (5'-DFUR) is a potent cytotoxic drug, which can be activated in cancer cells to give 5-fluorouracil ( $5^{\prime}$-FU). Ethidium is served as fluorescence report component, which is a mitochondria targeting fluorophore exhibiting strong fluorescence after binding with RNA or DNA. The last component is the ROS-triggered linker, which activates the prodrug in the presence of ROS. The release of 5'-FU in the presence of excess endogenous ROS induced the RNA or DNA damage in cancer cells, thus exhibiting enhanced targeted therapeutic efficacy ${ }^{53}$. 

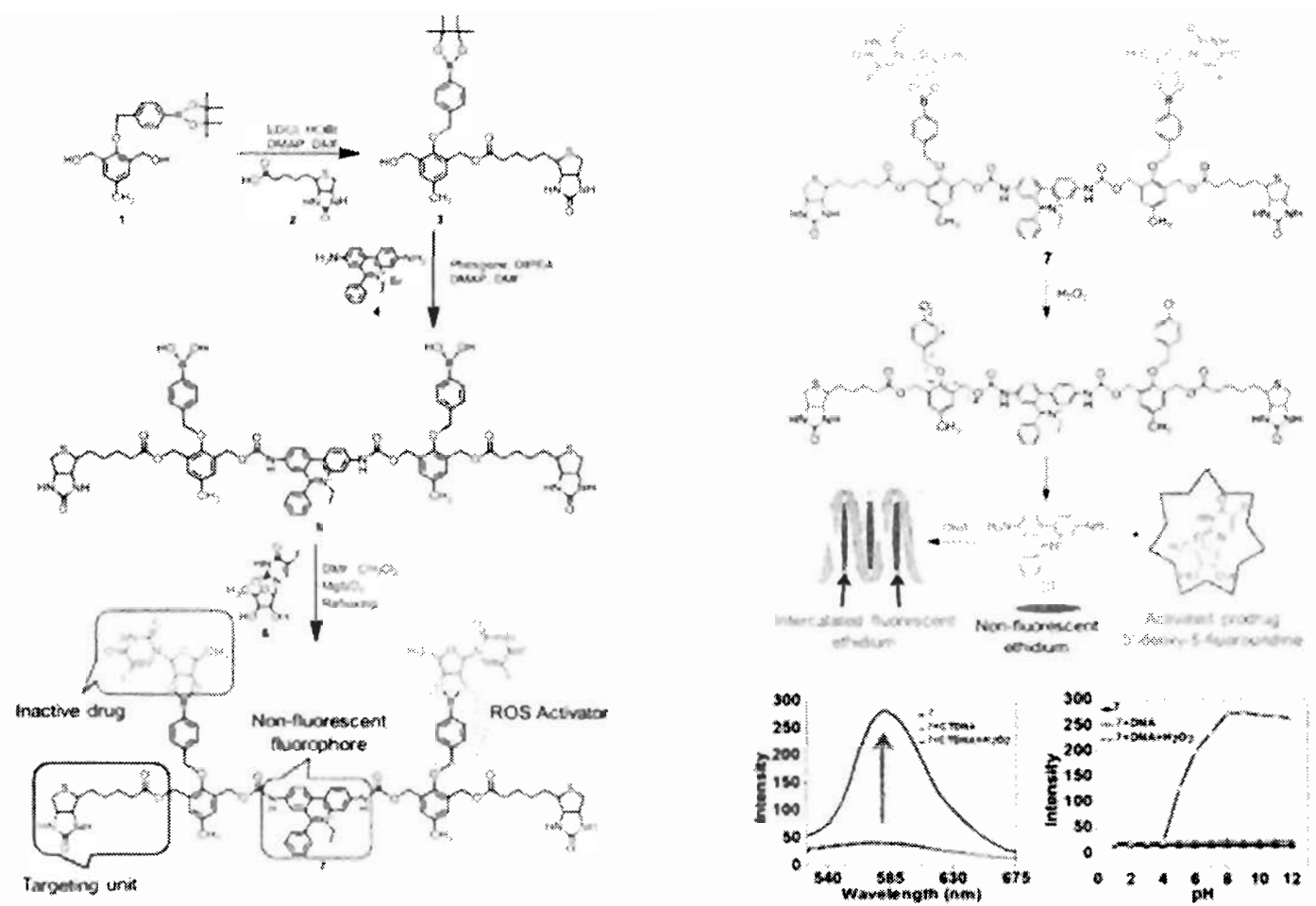

Figure 5 Schematic illustration of synthetic routes and hydrogen oxide activate release mechanism.

(Reproduced with permission from ref 53. Copyright 2014 American Chemical Society.)

It is well known that there is existence of complex redox balance in normal cells adjusting by various enzymes. However, in cancer cells, numerous evidences have shown that cancer cells are able to adapt to the high oxidative stress through the regulation the GSH concentration by activating antioxidant systems. Several efforts have been made to develop effective therapeutic techniques to increase the ROS level and kill the cancer cells specifically. Photodynamic therapy (PDT) is the treatment technique based on this principle. Upon irradiation of the light, the photosensitizers used in PDT can generate of ROS, resulting in apoptosis of cancer cells. There are other approaches to increase the ROS level, such as delivering the ROS generating agents to cancer cells directly or suppressing the antioxidant systems ${ }^{59-63}$. By utilizing the different redox balance between the normal cells and cancer cells, Lee and his 
group designed a sophisticated prodrug named QCA, which can be activated by $\mathrm{H}_{2} \mathrm{O}_{2}$ or acidic environments to release the active ingredient. As shown in Figure 6, the prodrug is composed four parts, a $\mathrm{H}_{2} \mathrm{O}_{2}$ trigger, an acidic trigger, a quinone methide, and a cinnamaldehyde. Uptake of such prodrug exposed it to highly acidic environment with an elevated amount of ROS level, resulting in the activation of prodrug. Upon activation, quinone methide and cinnamaldehyde moiety would be simultaneously released, acting as GSH scavenger and ROS-generating component in the cancers cells respectively. By upregulation of the ROS level and downregulation of the GSH level, the redox balance will be disrupted to achieve amplification of oxidative stress resulting in serious cell cytotoxicity. This prodrug is the first hybrid drug which can concurrently suppress the antioxidant systems and increase the ROS level, displaying excellent therapeutic performance both in vitro and in vivo ${ }^{64}$. 


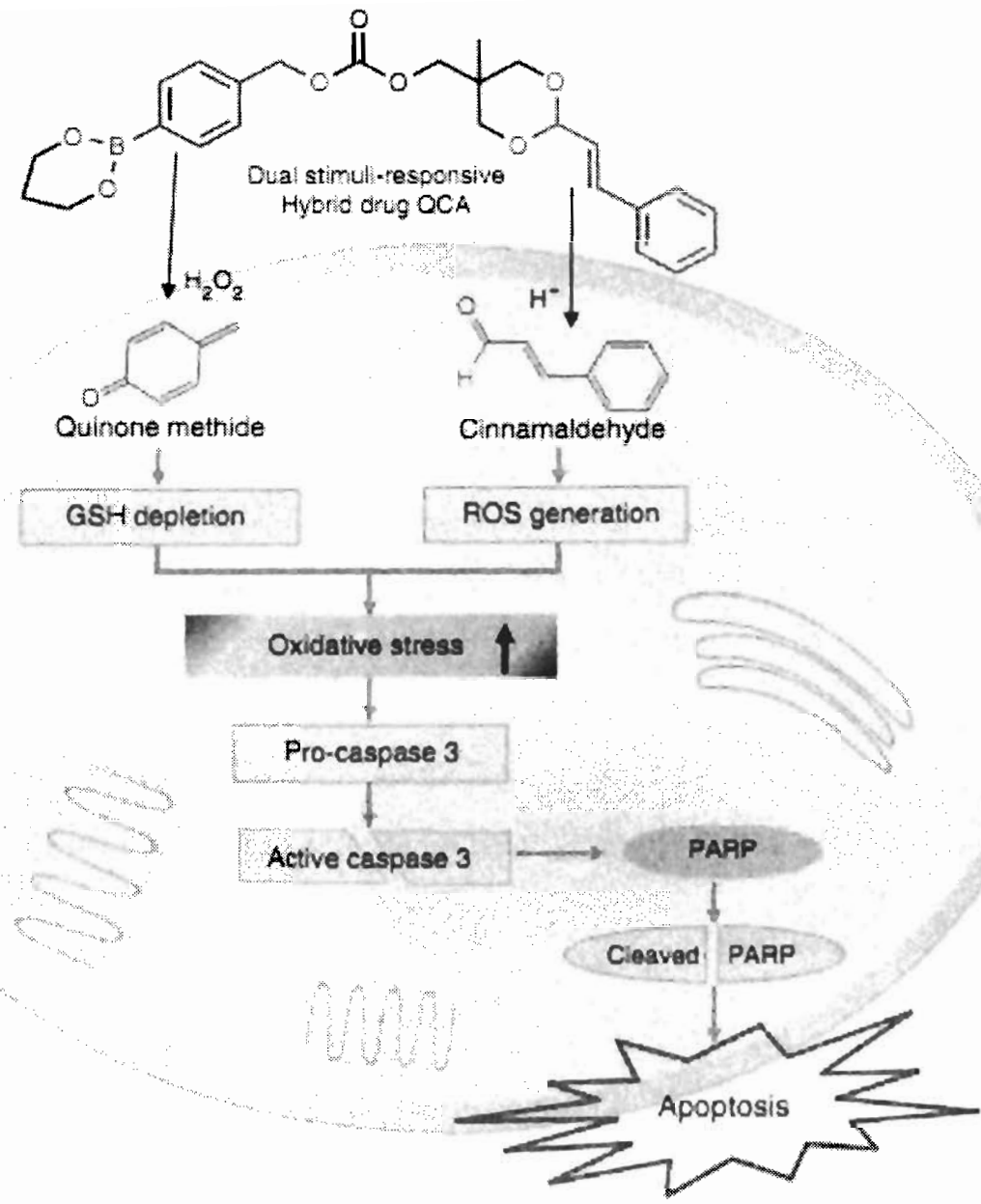

Figure 6 Schematic illustration of the structure and mechanism of prodrug. (Reproduced with permission from ref 64. Copyright 2015 Nature Publishing Group.)

\subsection{3. $\mathrm{pH}$ responsive prodrug.}

Because of high rate of glucose metabolism, the lactic acid concentration is higher in cancer cells than in normal cells, even in the presence of sufficient oxygen. Due to special vascular structure of the tumor, tumor shows poor perfusion property leading to accumulation of lactate in tumor sites, resulting in an acidic extracellular in tumor ( $\mathrm{pH}$ value about 6.5-6.9) than in normal tissue ( $\mathrm{pH}$ value about 7.2-7.4). Inside of the cancer cells, the $\mathrm{pH}$ value is much lower than the extracellular environments. This is especially so in some organelles, such as endosomes and lysosomes, the $\mathrm{pH}$ value can 
be observed at the range of 4.5-5.5. Thus, efforts have been made to design $\mathrm{pH}$ discrimination prodrugs in light of inherent acidic environments in tumor tissue $\mathrm{e}^{2,32,65-}$ 77.

The critical component in $\mathrm{pH}$ responsive prodrugs is the choice of the $\mathrm{pH}$ trigger. The $\mathrm{pH}$ triggered linker should display relative high stability in physiological conditions and can be cleaved under the acidic environment inside tumor cells. Among the several types of $\mathrm{pH}$-sensitive trigger, the hydrazone bond is the most popular trigger due to easy preparation and high sensitivity to $\mathrm{pH}$ changes. Doxorubicin (DOX) is widely used as active drug in design of acidic sensitive prodrug, because it contains a primary amine group in its active site, which allow easy modification of $\mathrm{pH}$ responsive hydrazone bond. In 2009, Chilkoti group reported a $\mathrm{pH}$ responsive polypeptide-doxorubicin conjugate linked by hydrazone bond. As shown in Figure 7, they used ELPs (elastin-like polypeptide) a type of artificial polypeptide as hydrophilic part which are composed of a Val-Pro-Gly-Xaa-Gly repeat unit derived from human tropoelastin exhibited good biocompatibility. A short segment grafted DOX as hydrophobic part, which is acidic sensitive due to presence of $\mathrm{pH}$-labile hydrazone bond. The chimeric polypeptides can form sub-100-nm-sized nanoparticles with near monodisperse distribution by spontaneously self-assemble. The prodrug nanoparticles exhibited four times higher maximum tolerance than the free DOX, and showed almost complete tumor ablation with a single injection ${ }^{78}$. 

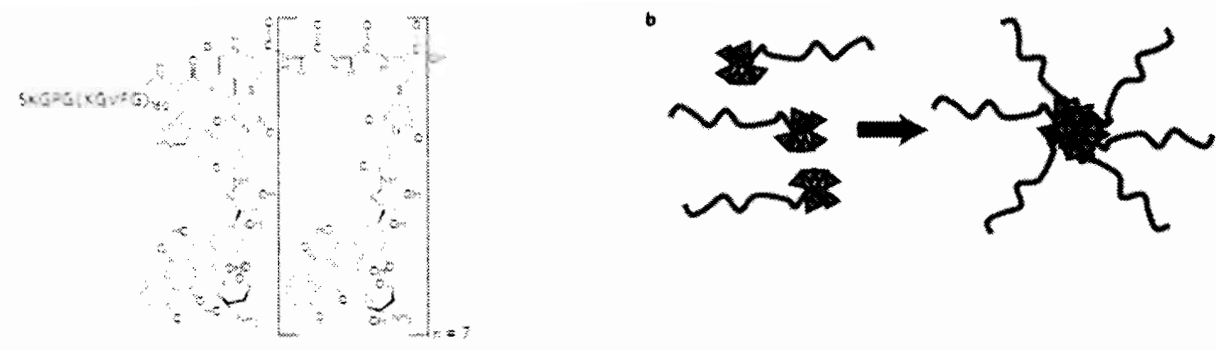

Figure 7 The structure of chimeric polypeptides prodrug and schematic illustration of nanoparticles formation. (Reproduced with permission from ref 77 . Copyright 2009 Nature Publishing Group.)

Zhang group reported a $\mathrm{pH}$ responsive prodrug as a theranostic platform for monitoring the release process in real time based on Förster Resonance Energy Transfer (FRET) strategy ${ }^{79}$. The prodrug was composed of four parts, a RGD peptide as tumor targeting segment, a fluorescence reporter coumarin, a $\mathrm{pH}$ sensitive linker hydrazone bond, and anticancer drug DOX. Under physiological conditions, the fluorescence of coumarin will be quenched by DOX, due to the FRET between coumarin and DOX. After uptake by tumor tissue through $\alpha_{v} \beta_{3}$ integrin mediated endocytosis, the hydrazone bond will be cleaved at the acidic tumor tissue, and the distance between the two fluorophores increases and thus disallow FRET between the two fluorophores to occur. The fluorescence of coumarin could thus be recovered, with a decrease in the fluorescence of DOX. Fluorescence changes can be thus be used to monitor the drug release process. This prodrug displayed quick on command release of DOX and rapid fluorescence recovery of the coumarin, which can be monitored in real time by fluorescence imaging technology. 


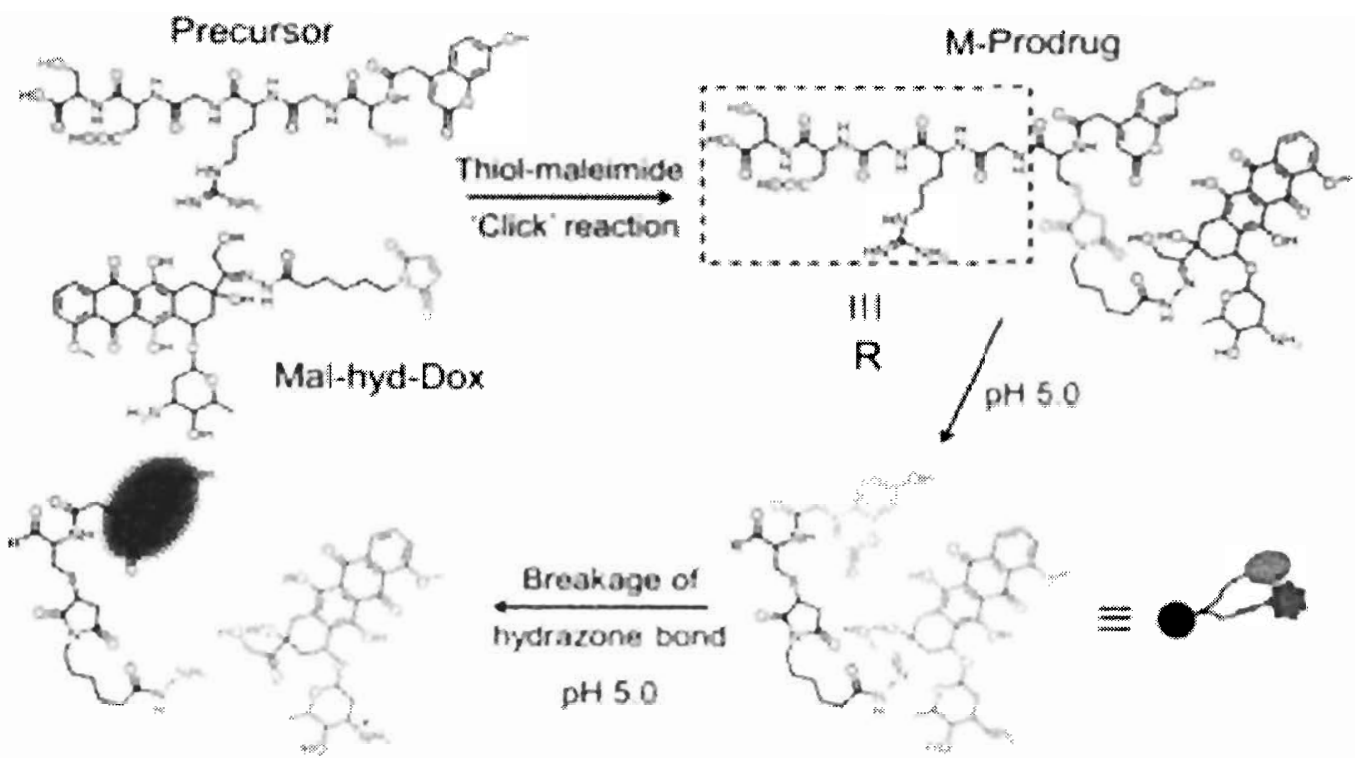

Figure 8 Synthesis route of the prodrug, and the mechanism of drug release and fluorescence changes under the acidic environments. (Reproduced with permission from ref 78. Copyright 2013 Royal Society of Chemistry.)

\subsubsection{UV triggered prodrug}

Photolytic cleavage has been regarded as a powerful approach to control the drug release due to excellent temporal and spatial accuracy under the photo irradiation. Many efforts have been made to introduce the photolytic groups into the prodrug design. The general strategy to synthesize photo active prodrug is to decrease the drug activity by blocking the active site of the anticancer drug, and this blocking groups can be removed under the light irradiation. Among the types of developed photolytic functional groups, o-nitrobenzyl derivatives have received considerable attentions in accounts of its efficient photolysis under the irradiation of UV light and its facile synthesis $^{80-85}$. 
In the past decades, numerous o-nitrobenzyl derivatives blocking prodrugs have been reported, especially in polymer prodrug. Using the two double active sites CPT derivatives (10-hydroxycamptothecin and 9-aminocamptothecin) as monomer to polymerization with o-nitrobenzyl derivatives, Chen group developed a polymeric prodrug with UV controllable release property ${ }^{86}$. As shown in Figure 9, the polymeric prodrugs were only composed of two parts, a trigger linker and a CPT drug, and showed high drug loading content. The polymeric prodrug could form nanoparticles with PEL polymer and exhibit $150 \mathrm{~nm}$ diameter with high drug loading content and high drug loading efficacy. The prodrug nanoparticles showed fast UV light responsive drug release, which could release $59 \%$ of the drug in 10 mins upon the irradiation of the UV light with a power of $40 \mathrm{~mW} / \mathrm{cm}^{2}$, while negligible drug release was observed without the UV light irradiation. In MTT assay, the polymeric prodrug showed 20 times lower $\mathrm{IC}_{50}$ values under the UV irradiation in HeLa cells than the same conditions without the UV treatment. 

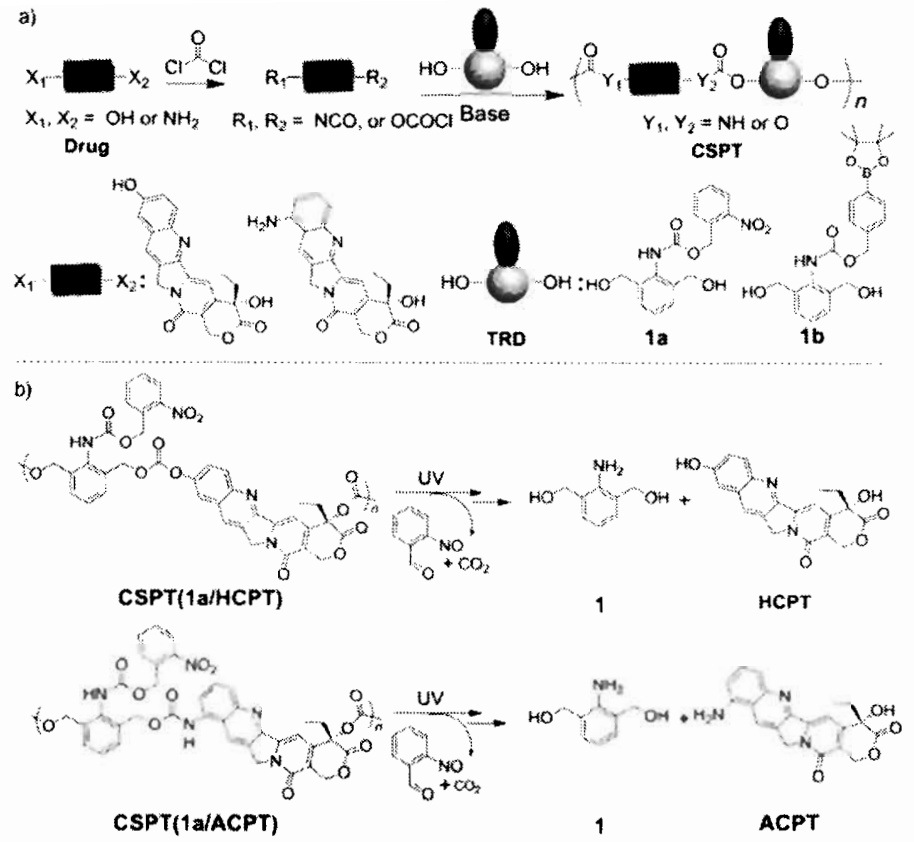

Figure 9 Synthesis of UV- and hydrogen peroxide responsive prodrugs, and the mechanism of degradation and release of the free drug under the UV light irradiation. (Reproduced with permission from ref 86. Copyright 2013 wiley)

Liu's group fabricated a triggered linker, composed of disulfide bond and a hydrazone bond, cross linked polymeric prodrug micelle named SCL micelle with a PCL grafted o-nitrobenzyl modified CPT as hydrophobic segment and folic acid functional coronas as hydrophilic segment ${ }^{87}$. As shown in Figure 10, the two types of co-polymers were mixed together and co-assembled to form a co-micelle, followed by shell cross-linking with a disulfide linker aldehyde groups. The obtained micelles can dissembled in the presence of acidic pH and high GSH environment, avoiding premature release of drug. The folic acid decorated surface provides targeting specificity via folic acid receptor mediated endocytosis. After UV irradiation, the prodrug micelles showed almost 10 -fold cytotoxicity enhanced than cytotoxicity in the dark. The SCL micelles displayed cancer cell targeting with multi-stimuli 
responsibility and exhibited excellent therapeutic performance upon irradiation of UV light.

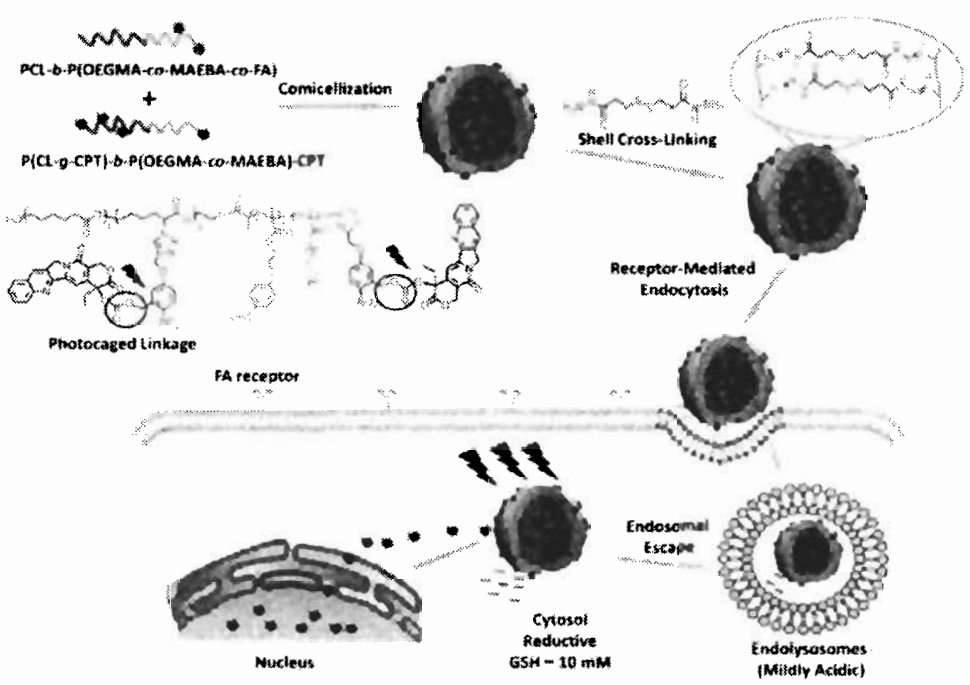

Figure 10 Schematic illustration of CPT polymeric prodrug, the process of preparation cross linking micelles, and UV trigger release in cancer cells. (Reproduced with permission from ref 87 . Copyright 2013 American Chemical Society.)

\subsubsection{NIR and two photon triggered prodrug.}

Due to great application potential and some inherent advantages including precise controlled release, high spatiotemporal resolution and stoichiometric cleavage under the irradiation of the light and non-contact mode of action, the photo-triggered prodrug have drawn great attention in the field of drug delivery and bio-imaging. However, some of the common photo trigger such as that of o-nitrobenzyl derivatives utilizes UV light for photolysis and thus suffers from several inevitable drawbacks, such as poor tissue penetration depth and phototoxicity. Suitable wavelength of light for deep tissue penetration should range from $600 \mathrm{~nm}$ to $900 \mathrm{~nm}$, which lies in the optical transparent window of tissue..$^{80,88-91}$ In order to achieve deeper tissue 
penetration and better therapeutic performance, Near-infrared (NIR) or two photon triggered prodrugs have been developed.

NIR is a light radiation with an optical window range from $750 \mathrm{~nm}$ to $1400 \mathrm{~nm}$ and show strong tissue penetration ability. Due to deep tissue penetration and low phototoxicity, NIR is an ideal light source to induce the photolysis. However, due to the inherent low energy of NIR light, it is challenging to find suitable functional linkers to construct the NIR-responsive photo active drug ${ }^{19,45,56,88,92-96}$ You et. al designed a NIR triggered PTX prodrug, which can release the original drug under the irradiation of $690 \mathrm{~nm}$ light ${ }^{97}$. They employed singlet oxygen sensitive linker to achieve drug release upon irradiation of NIR light. The prodrug was composed of three parts, a silicon phthalocyanine, an aminoacrylate linker and an anticancer drug PTX (Paclitaxel). SiPc (silicon phthalocyanine) was introduced into the system as photosensitizer, which can generate singlet oxygen under the irradiation of NIR light. Aminoacrylate linker is a singlet oxygen cleavable linker, which can be oxidized and release a carbon dioxide molecule followed by the original drug. The toxicity of PTX was effectively reduced because its active site was blocked after various modification. As shown in Figure 11, the photosensitizer can generate singlet oxygen under the NIR light irradiation, which will lead to oxidation of the aminoacrylate linker, resulting in drug release. This prodrug is the first PTX prodrug which can be activated by using NIR light and exhibited good therapeutic performance by combination of the photodynamic therapy and PTX chemotherapy. 

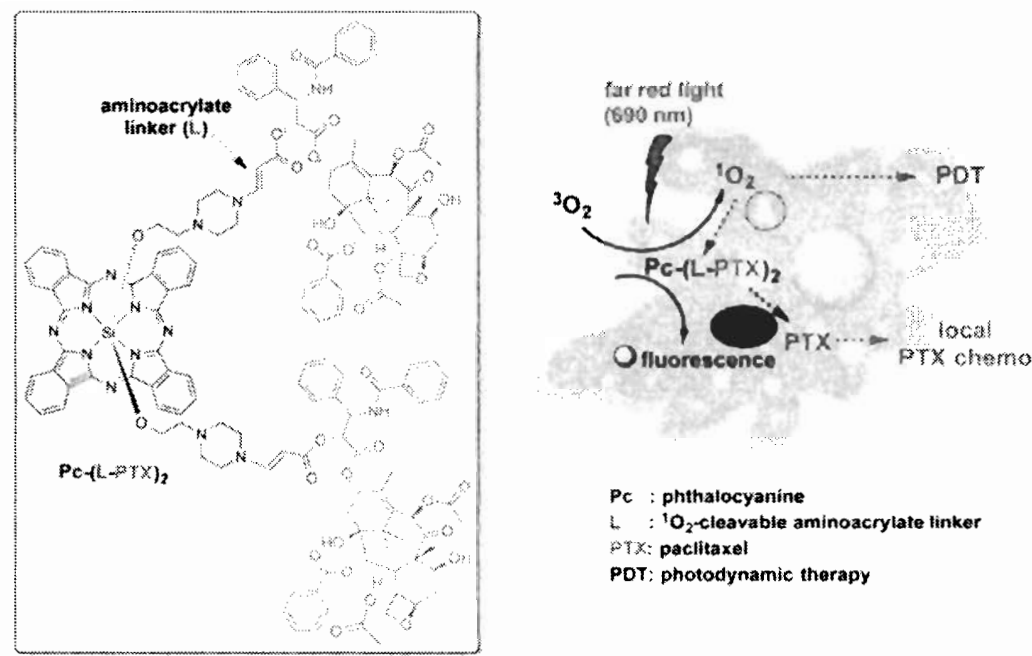

Figure 11 The structure of NIR responsive drug, and the schematic illustration of the work mechanism.

(Reproduced with permission from ref 97. Copyright 2016 American Chemical Society.)

As mentioned above, for the UV light, the shortcomings are its poor tissue penetration ability and phototoxicity, and for the NIR light, despite its excellent deep tissue penetration and negligible phototoxicity, the main limitation is the lack of NIR responsive linker. It is thus urgent needs to develop techniques which can overcome the drawbacks of the UV and NIR, namely to develop the techniques with numerous available linkers upon the excitation with NIR light.

Generally, when analyzing the optical absorption process, one photon absorption was observed for an atom in one transition, and it is a linear process. This holds true when the light source used is a weak energy source. Usage of light source (laser) with higher pulse and higher intensities resulted in new absorption process, whereby two photons are absorbed in a single atom for a single transition, known as the two photon absorption. Two photon absorption is a nonlinear optical process, which has drawn a lot of attention in bio-imaging due to special advantages of this process such as minimal scattering with background signal being suppressed. Numerous fluorophore 
with the two photon absorption property have been well investigated in photochemistry field and widely used in bio-imaging ${ }^{97-103}$.

Coumarin is a natural dye molecule and a widely used fluorophore with numerous derivatives because of easily modification of its molecular skeleton. Coumarin derivatives are series fluorophores and considered as outstanding candidate in fluorophore probe with excellent fluorescence properties such as high quantum yield, large stokes shift, and high photostability ${ }^{13,22,45,88,106-109}$.

The most interesting property of the coumarin is that it has a large two photon absorption cross section and suitable for two photon excitation. Zhu group reported a theranostic platform with two photon trigger prodrug by using coumarin as the fluorophore $^{81}$. As shown in Figure 12, the anticancer drug chlorambucil was conjugated with the coumarin derivative by ester bond, followed conjugation with silane to afford silane precursor. Then the precursor was grafted on the surface of the MSNPs (mesoporous silica nanoparticles) to give the prodrug functional nanoparticles. After uptake by HeLa cells, the active drug chlorambucil was observed to exhibit a controlled release in $40 \%$ within $2 \mathrm{~h}$ upon irradiation with the $800 \mathrm{~nm}$ NIR light and the maximum release could achieve almost $100 \%$ with the extending the irradiation time to $3 \mathrm{~h}$ by the power of $10 \mathrm{~mW} / \mathrm{cm}^{2}$. The MTT assay also exhibited toxicity enhanced along with increasing light irradiation time. 


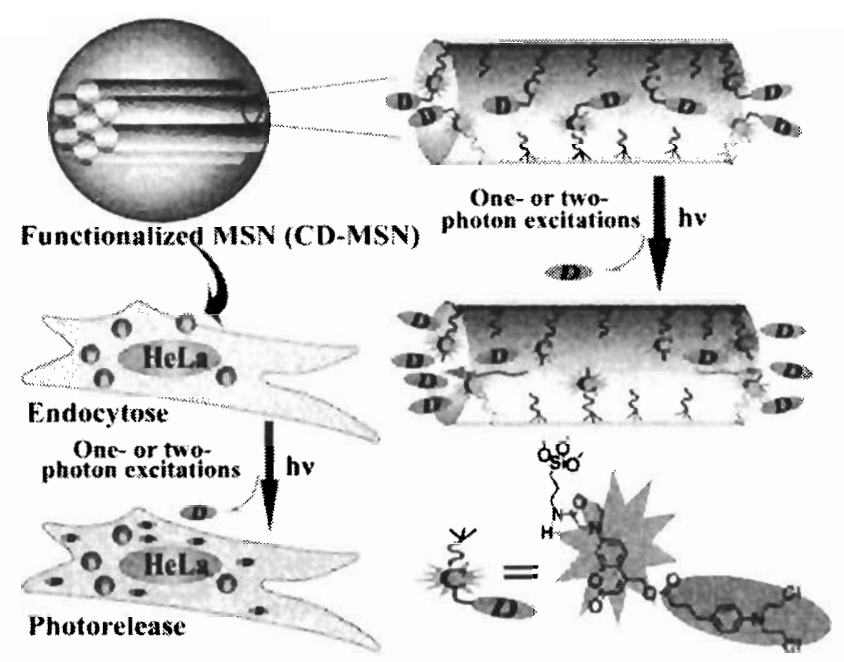

Figure 12 The schematic illustration of the prodrug nanoparticles and the photolysis for drug release under the one or two photon excitations. (Reproduced with permission from ref 81 . Copyright 2010 American Chemical Society.)

\subsubsection{Enzyme triggered prodrug}

Enzymes are great amount of biological macromolecular catalysts which play extremely important roles in all live systems. Most enzymes are proteins, and few of them are RNA. Enzymes as catalysts display a lot of distinguish advantages, especially in the living systems, including high specificity, non-reversible reaction, and high working efficacy at $37^{\circ} \mathrm{C}$, preferably monomeric, wide substrate tolerance, and its ample supply in biological system. Using enzymes to activate the drugs is very attractive, because it allows scientists to design and explore controlled release that are of high specificity, high efficacy in mild working environments. The specificity of the enzymes catalysis reaction offers the approaches to control prodrug activation to the tumor tissue, through construction of enzymes targeting cleavage block linker grafted anticancer drug. The wide substrate tolerance means that many types of prodrug 
combinations with different drugs and block groups can be accessed by activating with enzymes catalysis reaction. Due to abundance resource of enzymes, many enzyme-triggered drug constructions are available and have aroused great interest $^{37,110-112}$

DT-diaphorase is a cytosolic flavoenzyme which is a 2-electron transfer flavoprotein widely found in animal species, and catalyzes the conversion of various quinones into hydroquinones by using nicotinamide adenine dinucleotide phosphate (NADPH) or nicotinamide adenine dinucleotide (NADH) as the electron donor. ${ }^{13,89,113-116}$ Recently, literatures have revealed that there is an elevated level of DT-diaphorase in many tumor types as compared to normal tissues ${ }^{91}$. DT-diaphorase has been used as a trigger molecule to image the tumor tissues, and considered as a promising enzymes candidate to activated quinone modified anticancer drugs. As shown in Figure 13, Wu's group reported a theranostic prodrug which was composed by an anticancer drug CPT, a cleavable linker, and a DT-diaphorase-activatable quinone propionic acid segment. In this prodrug, CPT also served as the fluorescence reporter to monitor release, because the fluorescence of CPT is quenched by quinone propionic acid segment through PET (photoinduced electron transfer) process. After uptake by the cancer cells, the quinone propionic acid segment can be rapidly, at the same time, selectively reduced to corresponding hydroquinone, followed by its transfer to lactone form, and subsequently release free CPT coupled with fluorescence recovery. In comparison with normal cells, this prodrug displayed much higher cytotoxicity in DT-diaphorase overexpressed cancer cells. 


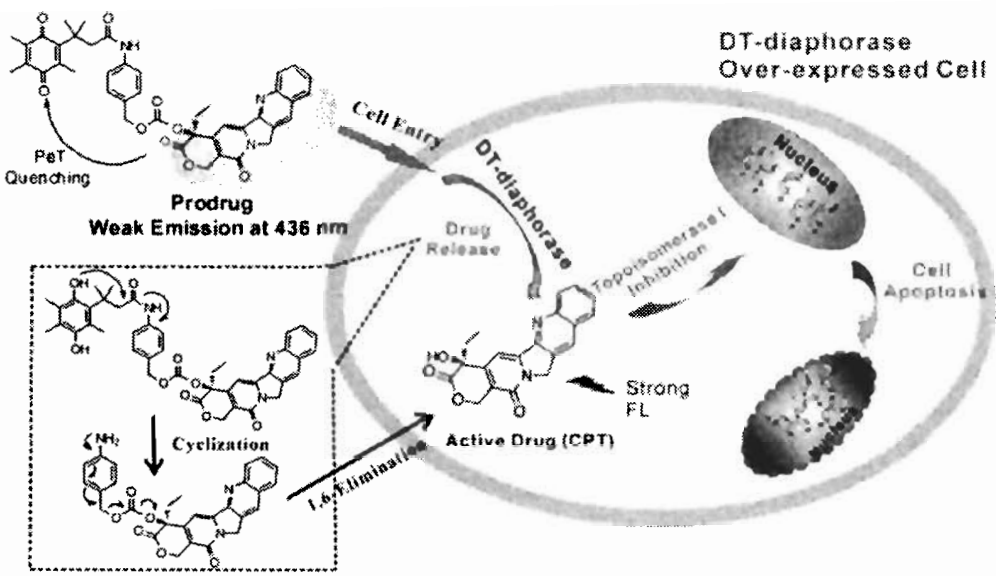

Figure 13 Schematic illustration of the structure of the enzymes activated prodrug and the mechanism of the drug release and DT-diaphorase detection after uptake by cancer cells. (Reproduced with permission from ref 91 . Copyright 2015 Royal Society of Chemistry.)

Antibody-directed enzyme prodrug therapy (ADEPT) is a promising strategy via enzymes targeting by modification with tumor associated antibodies and activating nontoxic prodrugs in cancerous tissues. B-galactosidase is a glycoside hydrolase enzyme which acts as catalyst to hydrolysis of $\beta$-glycosidic bond into a galactose and its organic moiety. $\beta$-galactosidase was found overexpressed in primary ovarian cancers which has been greatly investigated in ADEPT design $n^{4,117,118}$. The disadvantages of ADEPT include complex structures, high cost, and potential risk of immune response limit its application. Sbastien Papot and his coworkers reported a $\beta$-galactosidase responsive prodrug to overcome these limitations by avoiding the use of antibodies $^{119}$. As shown in Figure 14, the prodrug is composed of three components, a galactosidase trigger, a folic acid targeting ligand, and potent cytotoxic compound MMAE (monomethyl auristatin E). The prodrug is recognized by the folic acid receptor on surface of the cell membrane leading to receptor-mediated endocytosis, 
followed by intracellular drug release via enzyme-catalyzed mechanism. The two types of cancer cells, KB cells (Folic acid overexpressed cells) and A549 cells (folic acid negative expressed cells), were co-cultured and incubated with the prodrug. Results from MTT assay show that prodrug displayed much higher toxicity in KB cells than in A549 cells. In vivo experiments was done by injecting the prodrug in nude mice bearing $\mathrm{KB}$ xenografts via intravenous injections. As compared with the moderate inhibition of tumor growth of MMAE, the prodrug exhibited almost total inhibition of tumor growth. Above all, the prodrug showed well tolerated and low obvious toxicity at the tested dose, with all the mice survived after 31 days treatment. On the other hand, four out of seven mice succumbed for the MMAE group at the end of the study.
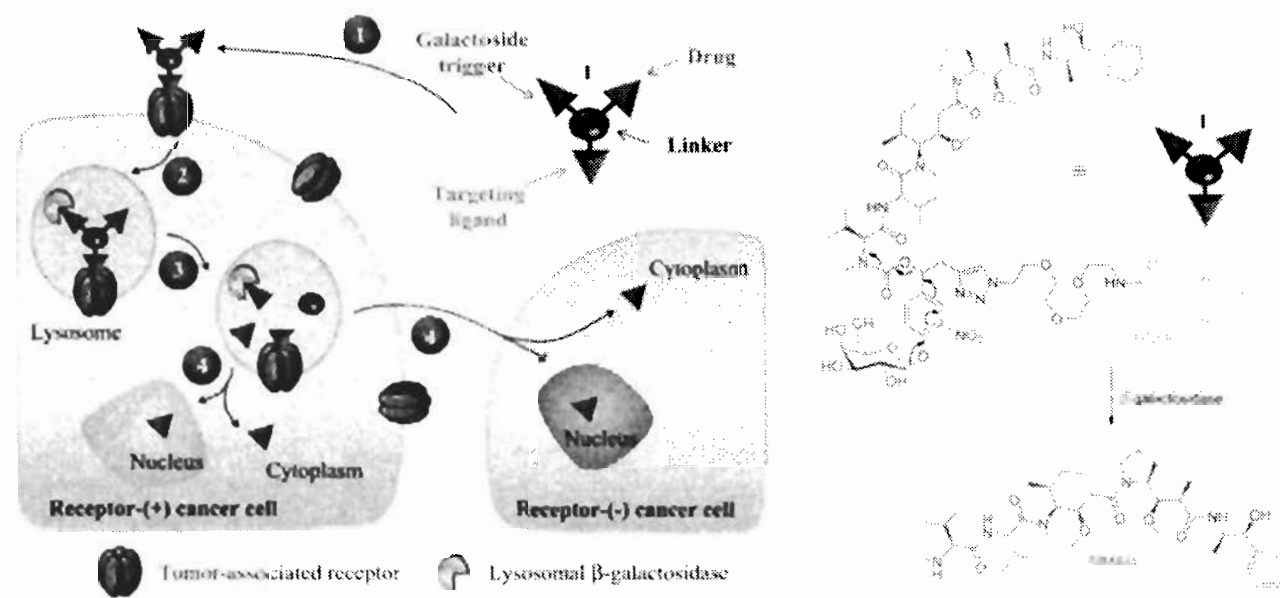

Figure 14 Schematic illustration of the principle of tumor targeting and structure of prodrug 1, and the b-galactosidase-catalyzed MMAE release mechanism. (Reproduced with permission from ref 119 . Copyright 2012 Wiley.) 


\subsubsection{Hypoxia triggered prodrug.}

As a consequence of tumor's rapid growth and slow apoptosis rate as compared to normal tissues, requirements for metabolism substrates including oxygen and glucose increase. Expansion of tumor volume also results in an increased distance between the tumor tissue and blood vessels, further decreasing blood supply. As a result, the oxygen concentration in tumor tissue is lower than in normal tissue, resulting in tumor hypoxia. Hypoxia is considered an important factor of the chemotherapy resistance and metastatic/aggressive form of cancer, resulting in low therapeutic efficacy. Thus development of hypoxia-activated prodrugs (HAP) which can be activated to release the effective therapeutic agents under the hypoxic conditions has drawn a lot of attention in cancer therapy field. The hypoxia triggered prodrug is composed of a hypoxia activated trigger and a chemo-therapeutic agent, which can remain intact in normal tissue with minimal cytotoxicity, and is only activated to release the anticancer drug in tumor tissue under the hypoxic environment. By using HAP, better therapeutic performance and reducing side-effects can be achieved in chemotherapy treatments of hypoxic tumor process. ${ }^{18,112,120,121}$

A tyrosinekinase is an enzyme which can transfer the gamma phosphate of ATP to amino acid residues in a protein, which lead to a conformational change resulting in protein malfunctioning. In some cases, the tyrosinekinase mutations occur uncontrollably and may contribute to initiation or progression of cancer. TKIs (Tyrosine kinase inhibitors) are small molecules inhibitor that are widely used in clinical cancer treatment which can compete with the ATP to bind with tyrosinekinase, 
leading to tyrosinekinase inactivation. The TKIs face serious drug resistance and side effects. Kowol and his group developed a HAP to inhibit the activity of the EGFR (epidermal growth factor receptor), a kind of tyrosinekinase ${ }^{122}$. As shown in Figure 15, TKIs like ligands were synthesized and coordinated with the cobalt to give the prodrug. The cobalt(III) compounds can be reduced into the cobalt(II) compounds because of $\mathrm{Co}^{\prime \prime \prime} / \mathrm{Co}^{\prime \prime}$ redox potential, and are designed as bioreducible prodrugs. The two different oxidation states display significant impact on the ligand lability, which can be affected by the cellular environments. Under the hypoxia tumor tissues, the cobalt(III) compounds immediately transform to $\mathrm{Co}^{\mathrm{Il}}$ complexes via bio-reduction, and ligand substitution could easily occur to selectively release the active ligands. Western blot analysis revealed that the expression of the EGFR was obviously inhibited under hypoxic conditions, as compared with normoxic conditions.
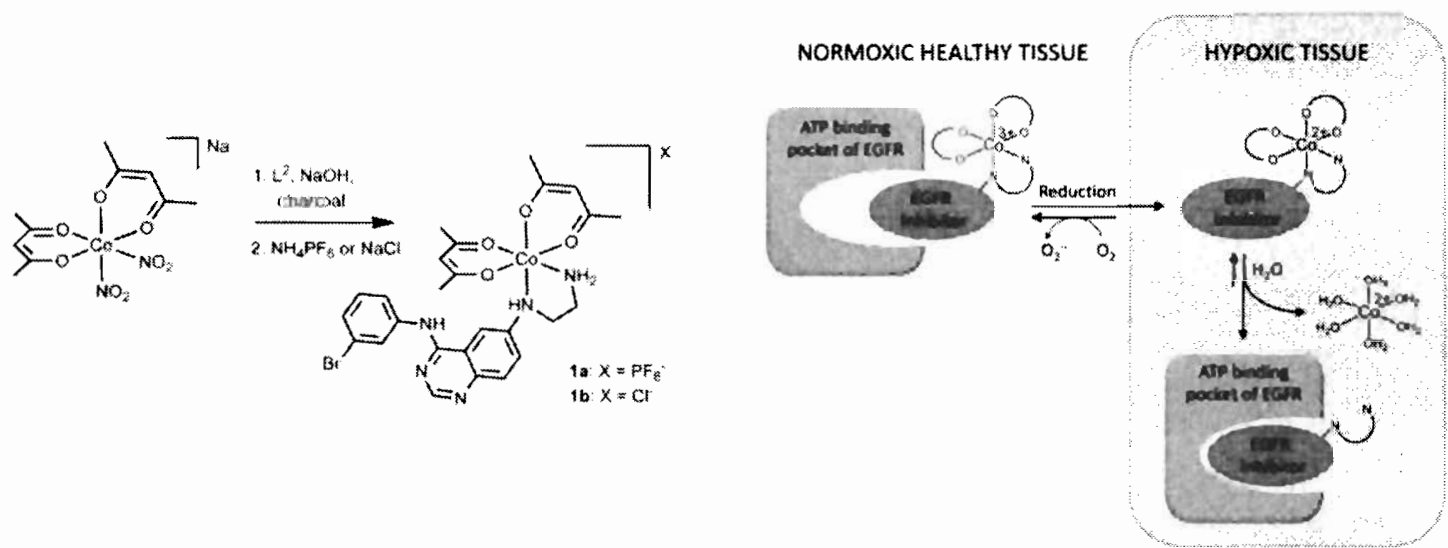

Figure 15 The Structure of the prodrug and the activation mechanism of inhibition of the EGFR.

(Reproduced with permission from ref 122. Copyright 2014 Wiley.)

Nitroaromatic functionalized units are widely used as a functional group for hypoxia-selective activation. The nitro group of the prodrugs can be reduced to activate the chemo-therapeutic agents under the hypoxic environments by cytochrome 
c reductase in presence of reduced NADPH. Hong and his team developed a theranostic prodrug, which is activated under the hypoxic micro-environments, and acts as a fluorescence reporter to image the tumor and monitor the release process in real time ${ }^{90}$. As shown in Figure 16, the prodrug has three constituents, the biotin targeting ligand, the nitroaromatic group, and the drug, SN38. The biotin acts as a targeting ligand to enhance the selectivity uptake of the cancer cells via its strong binding affinity to avidin receptors. The SN38 is a widely used anticancer drug and also plays a role as fluorescence reporter. The nitroaromatic unit remains intact in normal tissue without toxicity, and can be reduced to activate the drug release in hypoxic tumor tissues. After treatment with nitroreductase, fluorescence recovery was observed due to release of the free SN38 drug. In vitro experiments also show that the prodrug exhibited much higher cell toxicity under the hypoxic conditions, as compared to that of normoxic conditions. In in vivo experiments, substantial enhanced fluorescence of SN38 was observed in tumor tissues as compared with other organs including liver, lung, kidney, heart, and spleen, which shows that prodrug exhibit high tumor accumulation selectivity. After intravenous injection, the tumors treated with the prodrug showed obvious volume shrinkage and slowing growth, on the other hand, in the mice treated with free SN38, continuous growth was observed. 
A
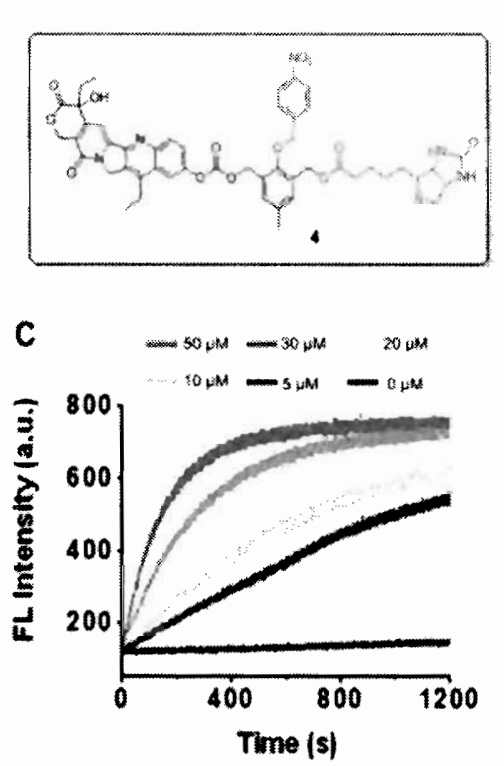

B

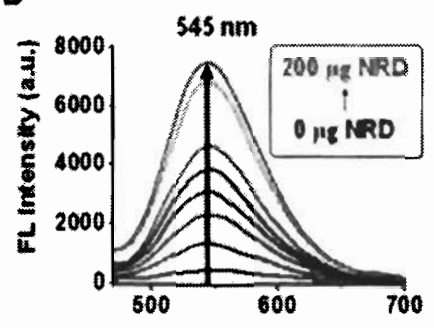

D Wavelength (nm)

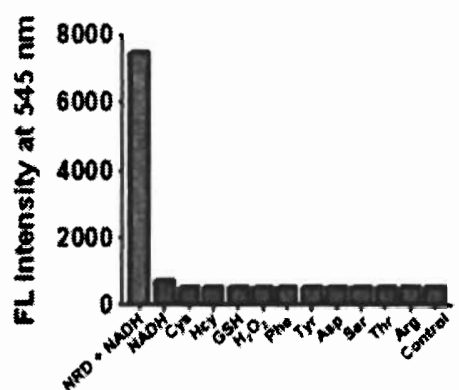

Figure 16 The structure of the hypoxic responsive prodrug, and the fluorescence changes after treatments with the nitroreductase of various concentrations. (Reproduced with permission from ref 90. Copyright 2016 Elsevier.)

\subsection{Supramolecular chemistry and its biomedical application}

In 1987, Nobel Prize of chemistry was awarded to three scientists, J. M. Lehn, C. J. Pedeseon, and D. J. Cram for their breakthrough contribution in supramolecular chemistry. After near 30 years of development, achievements have been made in supramolecular chemistry, both in theory and application. ${ }^{56}$ Just this year, the Nobel prize in chemistry was awarded to three chemists in supramolecular-related field, B. Feringa, J. F. Stoddart, and J. P. Sauvage, for their contribution of the design and synthesis of molecular machines. The interactions responsible for the spatial organization of molecules in supramolecular chemistry often involve non-covalent interactions including hydrogen bonding, ${ }^{123}$ van der Waals' force, ${ }^{124}$ electrostatic interaction, $^{125} \pi-\pi$ stacking interaction, ${ }^{107}$ electrostatic interaction, and 
hydrophilic/hydrophobic interaction. ${ }^{22}$ The birth of the supramolecular concept has since gained interests of scientists from various fields, including chemistry, biology, and materials. In the recent years, numerous DDS have been developed based on supramolecular concept.

\subsubsection{Hydrogels or supramolecular hydrogels for drug delivery}

In traditional concept, hydrogels are three dimensional, chemical or physical cross linked networks of natural and synthetic polymer chains, which swell upon contact with water to maintain their structures. ${ }^{36,126,127}$ Most widely used polymers to prepare hydrogels are natural polymers, including polysaccharides (hyaluronic acid, chitosan, cellulose, alginate etc.), polypeptides (fibrin, gelatin, collagen etc.), and proteins, which inherently have the high biocompatibility and the low toxicity. ${ }^{128-130}$ The natural polymeric hydrogels have plenty of important biomedical applications, such as drug delivery, biofabrication, and tissue engineering. The small anticancer drugs are easily trapped into the three dimensional networks of the hydrogels, and sustainable or controllable release under physiological conditions. ${ }^{131-134}$ Shoichet and his group used injectable hydrogels to deliver tunable growth factor for tissue engineering. ${ }^{135}$ As shown in Figure 17, methyl cellulose was modified with SH3-binding peptide which was introduced through EDC coupling followed by thiol- click reaction, and mixed with unmodified hyaluronan acid, which can efficiently decrease the gelation temperature of the methyl cellulose and endue the hydrogels injectable ability due to the excellent shear-thinning property of hyaluronan, to afford a fast gelling blend polymer. The methyl cellulose was grafted with two different SH3-binding peptide 
with different binding affinities to SH3: weak binder peptide with the sequence of KPPVVKKPHYLS and strong binder peptide with the sequence of KKTKPTPPPKPSHLKPK. The N-terminus of the SH3-binding peptides which composed of three glycine residues exhibited binding ability of the SH3-rhFGF2 via protein-peptide recognition. In vitro experiments, the three hydrogels, HAMC (the hydrogels of the methyl cellulose and hyaluronan without the peptide binder), HAMC-weak binder (the hydrogels of the hyaluronan and methyl cellulose modified with the weak peptide binder), and HAMC-strong binder (the hydrogels of the hyaluronan and methyl cellulose modified with the strong peptide binder), displayed different release rate under the mimicking in vivo environment. By using the hydrogels strategies, tunable and in linear release of protein can be obtained based on affinity difference, which also can be used in various proteins and materials.

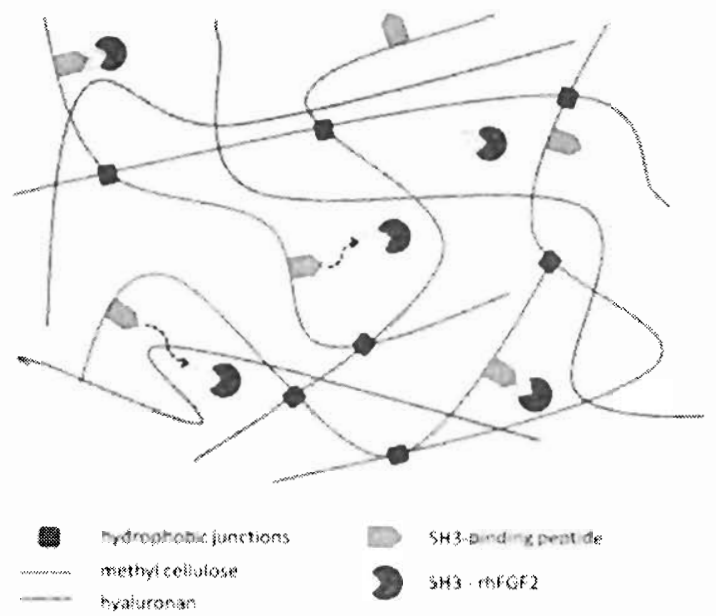

Figure 17 Schematic illustration of the compositions of the hydrogels, and the controlled release of SH3-rhFGF2. (Reproduced with permission from ref 135. Copyright 2012 American Chemical Society.) 
Gels prepared by the small molecules are called supramolecular gels, which usually have long fiber-like morphology formed by heating the gelator to dissolve it completely in the solvents and cooling to room temperature. The supramolecular gels prepared with water are called supramolecular hydrogels, the most used gelator of which are short peptides. The supramolecular hydrogels show great promising applications in tissue engineering and drug delivery, due to inherent low toxicity, biodegradability, and various external stimuli responsibility including light, heat, electric, enzyme, and $\mathrm{pH}^{56,136,137}$

Anticancer drugs modified with short peptides can afford the peptide prodrugs, and generate the hydrogels under specific conditions. Xu group reported an enzyme stimuli taxol-bearing peptides with the sequence of phosphorylated naphthalene-PhePhe-Lys-Tyr, which can initiate the self-assembly in water to form the nanofibers and spontaneously give a supramolecular hydrogel after treatments with the ALP (alkaline phosphatase) as shown in Figure 18. They found that both the cell toxicity of the taxol-bearing precursor and the hydrogelator displayed similar toxicity compared with free taxol, which indicated the drug's activity remained in presence of the ALP. The work provides a new way for in situ formation of supramolecular hydrogels, which exhibit sustainable release of the therapeutic agents. ${ }^{138}$ 

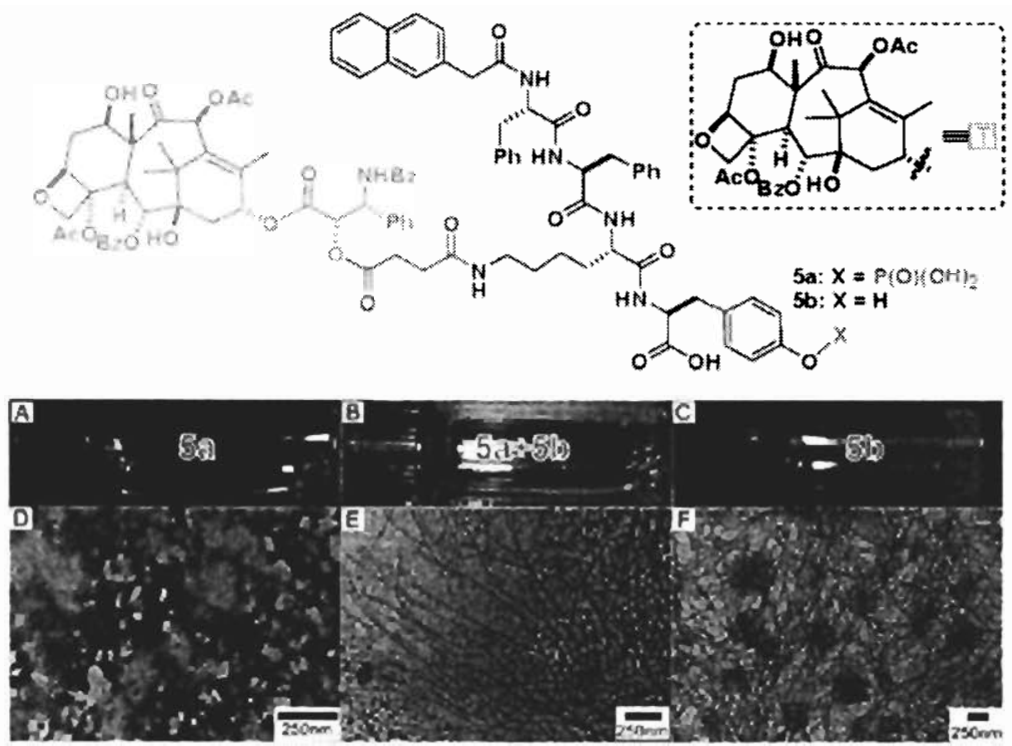

Figure 18 The structure of the taxol-bearing peptides. The morphology and appearances changes in the absence and upon treatments of the ALP (alkaline phosphatase). (Reproduced with permission from ref 138. Copyright 2009 American Chemical Society.)

\subsubsection{Liposome and polymersome for drug delivery.}

Liposome is a type of drug carrier that are most widely used and investigated in the field of nanomedicine after it is first described in 1960s by Bangham. With five decades of development, the liposome has been applied in various fields including the cosmetic, food, and pharmaceutical industry. Liposomes are particles with spheres like morphology, consisting of a lipid bilayers and an aqueous core within the bilayers composed of phospholipids. As drug carrier, the liposomes display several advantages which include improvement in the solubility of the drugs, protection of the drugs from degradation, reduction in toxicity of drugs, alleviation of side-effects arise from direct consumption of potent drug, improvement in the therapeutic efficacy, biocompatible, biodegradable and enables facile modification. ${ }^{117,139-142}$ 
In a work by our group, liposome was used as a carrier to load two different therapeutic agents for NIR triggered dual therapy. ${ }^{92}$ IR825 is a NIR dye with cationic heptamethine indocyanine structure, and has strong absorption in NIR region with negligible fluorescence quantum yield, which allows it to be an excellent candidate as photothermal therapeutic agent. As shown in Figure 19, IR 825 was loaded in the bilayer of PEG-protected DPPC/DSPC thermoresponsive liposome, and widely used chemotherapeutic drug DOX was loaded into the aqueous core of the liposome. Upon irradiation with the NIR laser for 4 mins, the temperature of the experiment setup was increased to $58{ }^{\circ} \mathrm{C}$, exceeding the phase transition temperature $\left(39-42{ }^{\circ} \mathrm{C}\right)$ of the thermoresponsive liposome and thus initiates the DOX release. The therapeutic performance of the liposomal system was tested in vivo by using zebrafish embryos as model animals, and the substantial tumor growth suppression and tumor ablation were observed in the presence of the NIR irradiation.

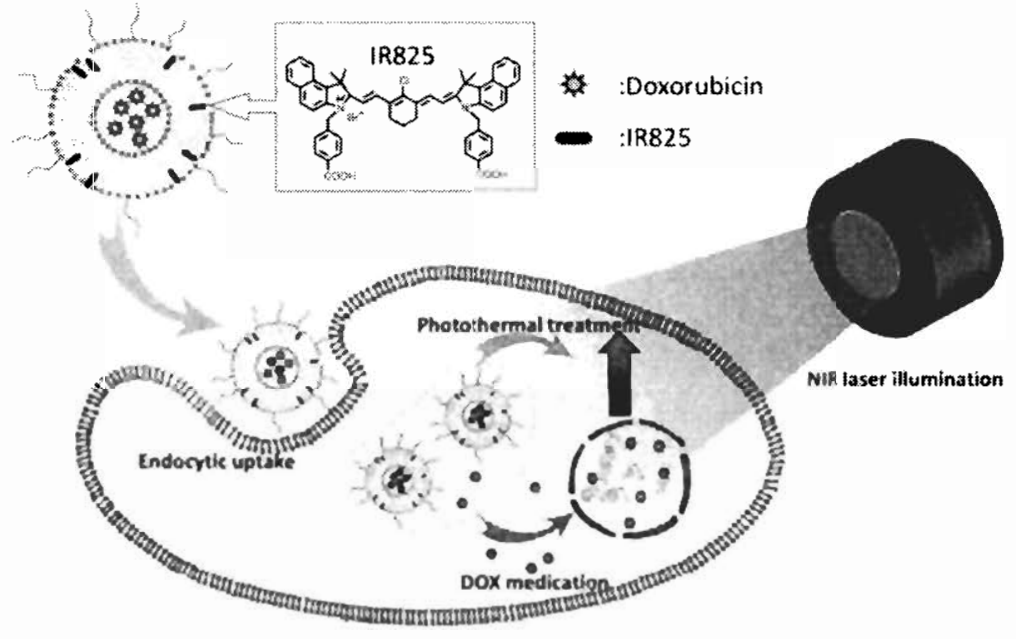

Figure 19 Schematic illustration of structure and the mechanism of the light trigger photo-thermat combined with chemotherapy. (Reproduced with permission from ref 92. Copyright 2015 Wiley.) 
Polymersomes are a type of artificial vesicle-like nanoparticles assembled by synthetic amphiphilic block copolymers. Similar to liposomes, the typical polymersomes have a spherical like morphology with an aqueous core surrounded by bilayer membrane. In the past decades, polymersomes have attracted lots of attentions as drug carriers, due to their colloidal stability, tunable surface functions, and efficient drug loading ability. Recently, efforts has been done to focus on developing "smart" biodegradable polymersomes that are external stimuli responsive, enabling the release of drugs in a controlled manners. ${ }^{107,143-147}$

Very much of the polymersomes with external stimuli properties, including light, heat, electricity, sonication, redox, and $\mathrm{pH}$, have been developed for controlled drug delivery or bio-imaging. A research group led by Liu used spiropyran as phototrigger to fabricate a photoswitched reversible polymersome and tested the photocontrolled release in cell level. ${ }^{107}$ Spiropyran is a widely used reversible photo switch, which undergoes isomerization between hydrophobic forms (spiropyran) and hydrophilic forms (zwitterionic merocyanine) under the different wavelength irradiation. As shown in Figure 20, the amphiphilic copolymer was composed of two blocks, PEO as hydrophilic fragment and PSPA as hydrophobic fragment, and it was assembled to form polymersome due to inherent amphiphilic properties. The polymersome underwent the photoisomerization upon irradiation with different wavelength light, resulting in microenvironmental polarity change. The polymersome structure still maintained the vesicle-like structure after polarity reversible changes. For in vitro 
experiments, results show that the polymersome exhibited photo controlled release under the alternated UV-Visible light irradiation.

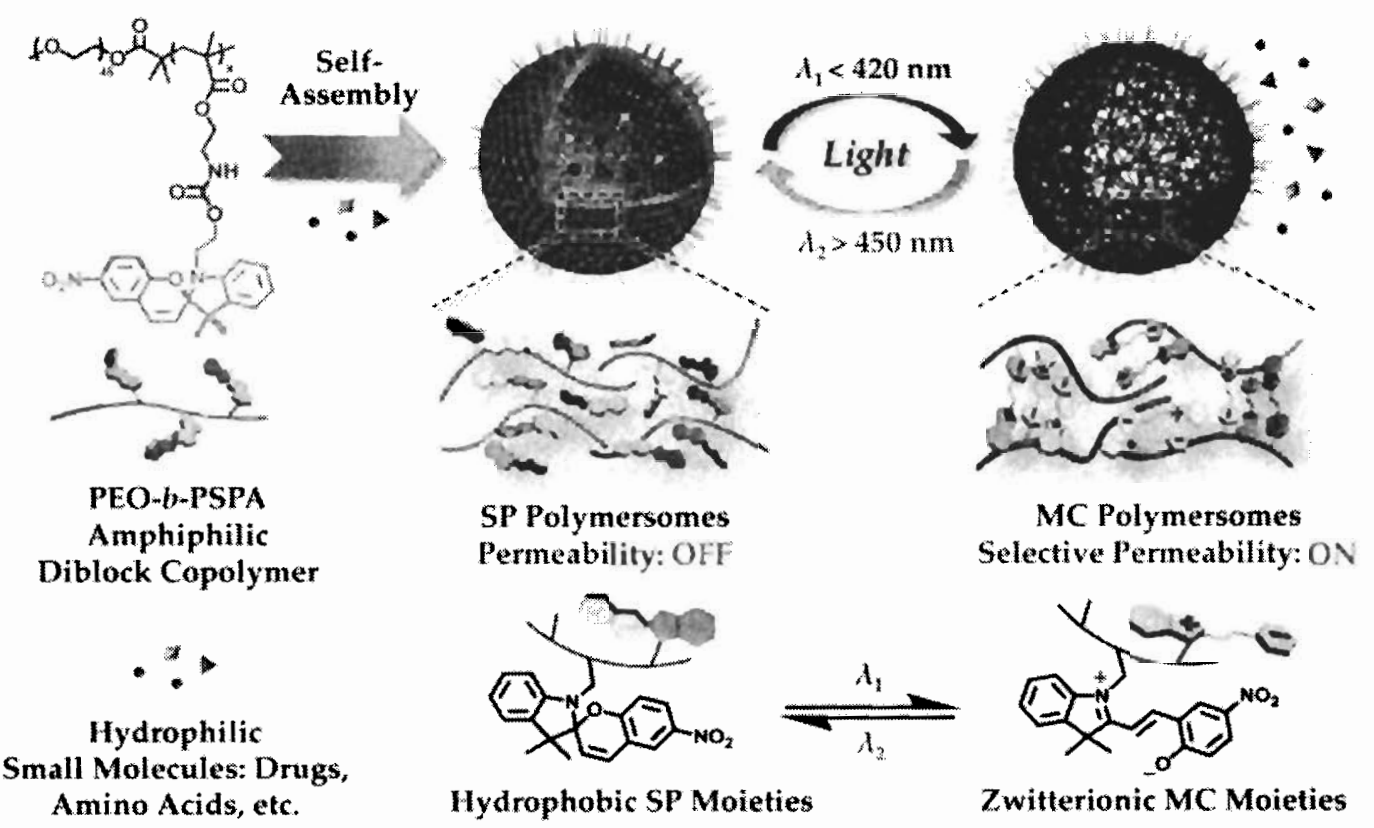

Figure 20 Schematic illustration of the structure of the amphiphilic copolymer, and the mechanism of photo-switched reversible self-assembly. (Reproduced with permission from ref 107. Copyright 2015 American Chemical Society.)

\subsubsection{Host-Guest chemistry and applications in biomedical}

Host-Guest chemistry is a branch of supramolecular chemistry, which focus on investigating of the interactions between macrocyclic host molecules and guest molecules. ${ }^{56,148-152}$ The two functional segments can be integrated together via host-guest inclusion, forming host-guest inclusion complex or supramolecular aggregates. During the past decades, three macrocyclic host generations have been developed, including the first generation host crown ether, the second generation host 
cyclodextrin, ${ }^{153}$ and the third generation host cucurbit[n]urils, calix[n]arenes, and pillar[n]arenes. ${ }^{154,155}$

\subsubsection{Host-guest inclusion complex between macrocyclic molecules and drugs}

Cyclodextrins (CDs) are a series of macrocyclic molecules with $\alpha-1,4$ glycosidic bond to form the oligosaccharides. It is produced from enzyme triggered starch degradation, and widely used as drug addictive approved by United States Food and Drug Administrator (FDA). CDs with different number of glucose units are called $\alpha$-, $\beta$-, and $\gamma$-CD, which refer to 6,7 , and 8 glucose units, respectively. The primary hydroxyl groups are located in one side of the ring construction of the primary side of the CDS; the secondary hydroxyl groups are located in another side of the ring construction of the CDs. The outside walls of the CDs are constituted by the hydroxyl groups pointing outwards, while the hydrogen atom at the $\mathrm{C} 3, \mathrm{C} 5$ and oxygen atom of the glycosidic bond are oriented towards inside of the CDs, resulting in high density of the electron clouds. Due to the special bonding and atom orientations of the glucose monomers in CDs, it possesses hydrophilic surfaces, coupled with hydrophobic cavities. Therefore, CDs are able to complex with various hydrophobic organic, inorganic, and biomolecules to form selective host-guest inclusion complex or even supramolecular aggregates. ${ }^{136,156-159}$

Approved by FDA, the drugs-CDs complexes are commercially available, and the CDs can efficiently improve the solubility of the drugs, protect the drugs from degradation, and enhance the circulation performance of the drugs. Due to mature commercialized, only a few of the literatures are reported in preparation of the 
drugs-CDs complexes in recent years. Velty and his team members used the HA grafted with cyclodextrin to encapsulate the anticancer drug PTX as illustrated in Figure 21. The cyclodextrin-PTX inclusion complex was self-assembled into a hollow capsule structure with the host-guest ratio of 1:1, and exhibited targeted suppression of the breast cells growth ${ }^{160}$.
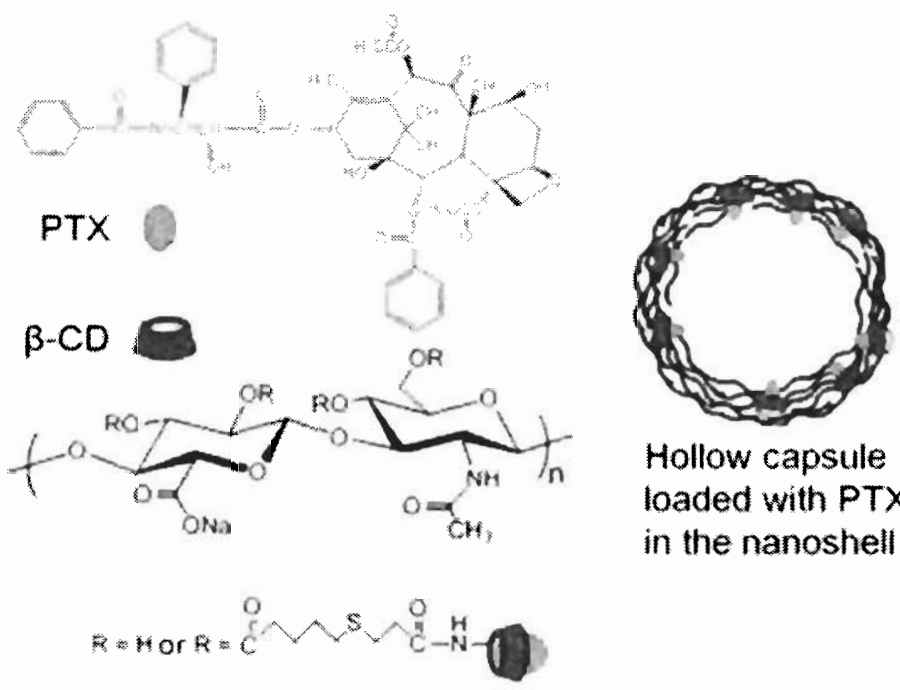

Hollow capsule loaded with PTX in the nanoshell

Figure 21 Schenatic illustration of the hollow capsule structure formed by cyclodextrin-PTX complex.

(Reproduced with permission from ref 160 Copyright 2012 American Chemical Society.)

Cucurbit[n]urils (CBs) are produced by condensation of the glycoluril and formaldehyde under the acidic catalysis conditions, which are first synthesized at 1905 with its full structure characterized until 1981 by Mock et. al. ${ }^{161}$ Just like CDs, the $\mathrm{CBs}$ are also consist of series members, including $\mathrm{CB}[5], \mathrm{CB}[6], \mathrm{CB}[7])$ with $\mathrm{CB}[8]$, and $\mathrm{CB}[10]$ as synthesized by Issaacs ${ }^{162}$. CBs have a pumpkin like shape with hydrophobic cavity surrounded by the hydrophilic carboxylated rims. Due to the cavity of the CBs surrounded by the carbonyl, the CBs contain numerous cation 
binding sites, which allow the CBs to encapsulate the molecules with positive charge though ion-dipole interactions and hydrogen bond. Compared with the other macrocyclic molecules, the $\mathrm{CBs}$ have a much more rigid structure, which give the CBs high affinity towards some guest molecules with suitable size and positive charge. ${ }^{163-165}$ The affinity constant between the $\mathrm{CB}[7]$ and the ferrocene was reported to $10^{12} \mathrm{M}^{-1}$, and highest affinity constant reportedly reached $10^{15} \mathrm{M}^{-1}$.

Because of the strong affinities and good biocompatibility, the CBs have also been used as drug carriers to enhance the solubility of the hydrophobic drugs. Issaacs' group have extensively investigated the drug solubility enhancement via using various CBs as drug carriers. They developed a series of acyclic cucurbit[n]uril molecular containers to improve the solubility of the poorly soluble pharmaceuticals. ${ }^{166}$ As shown in Figure 22, two kinds of the acyclic cucurbit[n]uril with different glycoluril units and end groups exhibited significant solubility improvement of the ten poorly soluble drugs with various structures, especially, for the paclitaxel, the enhancement observed 2750 fold improvement. 


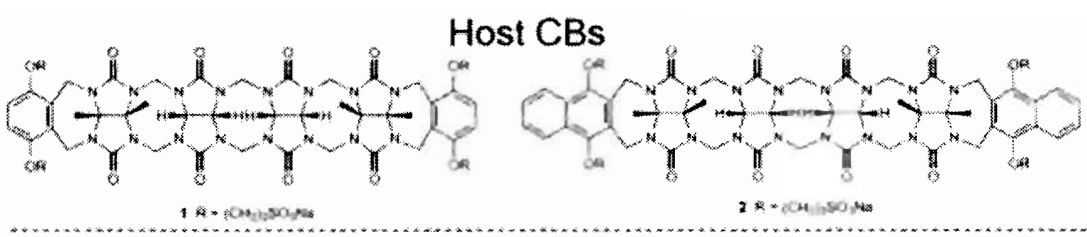

\section{Guest Drugs}
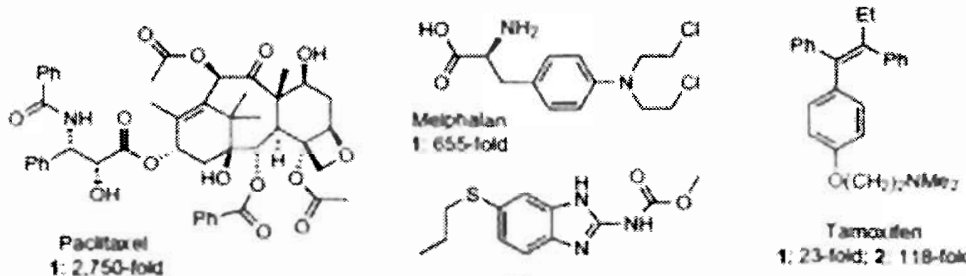

Abendizon

Tamoxthen

1: 23-10id: 2 118-80id

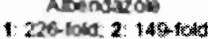

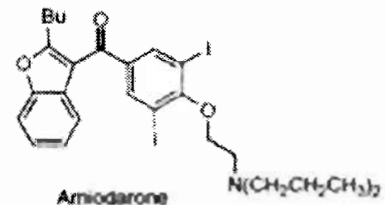

1: 207 -10010, 2 3earold
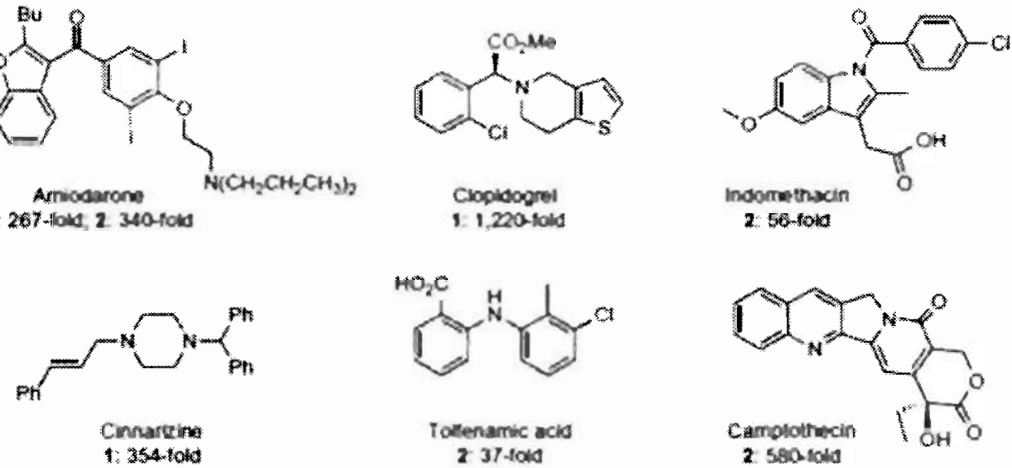

Figure 22 The structures of the acyclic $C B[n]$ containers 1 and 2 , and the enhancement in solubility of the poorly soluble drugs. (Reproduced with permission from ref 166. Copyright 2012 Nature Publishing Group.)

\subsubsection{Rotaxane and Polyrotaxane-Based Supramolecular nanoparticles.}

MSNPs (mesoporous silica nanoparticles) are widely used in drug delivery as drug carrier, due to lots of advantages, including thermal stability, large loading capacity, adjustable pore size, and easy surface functionalization. To prevent the pro-leakage of the drug molecules, many types of gate linkers have been introduced to provide the controlled release property of the MSNPs, such as disulfide, rotaxanes, saccharides, and polyetherimide. Among diverse smart linkers, rotaxane based linkers have 
garnered considerable interest due to various excellent external stimulus responsive ability. ${ }^{50,167-171}$

Our group reported a rotaxane functionalized MSNPs for thermal and photo dual triggered drug release. ${ }^{68,172}$ As shown in Figure 23, the rotaxane composed of the two moieties, $\alpha-C D$ ring act as a gate to prevent drug leakage, an azobenzene derivative acts as an axle and photothermal responsive trigger. At the initiation state, the azobenzene was at its trans-form, the $\mathrm{CD}$ ring is located at the trans azobenzene position which allows the sufficiently drug loading in the mesoporous silica layer. Upon irradiation with the UV light, the drug molecules are sealed within the pores because the azobenzene transform to its cis-form. For in vivo experiments, coumarin was chosen as the model drug, and the zebrafish was served as model animal. Under $37{ }^{\circ} \mathrm{C}$ water, after injection of the MSNPs, significant improvement in heartbeat was observed, proving that the heart failure of the zebrafish was greatly relieved via the thermal triggered therapy.
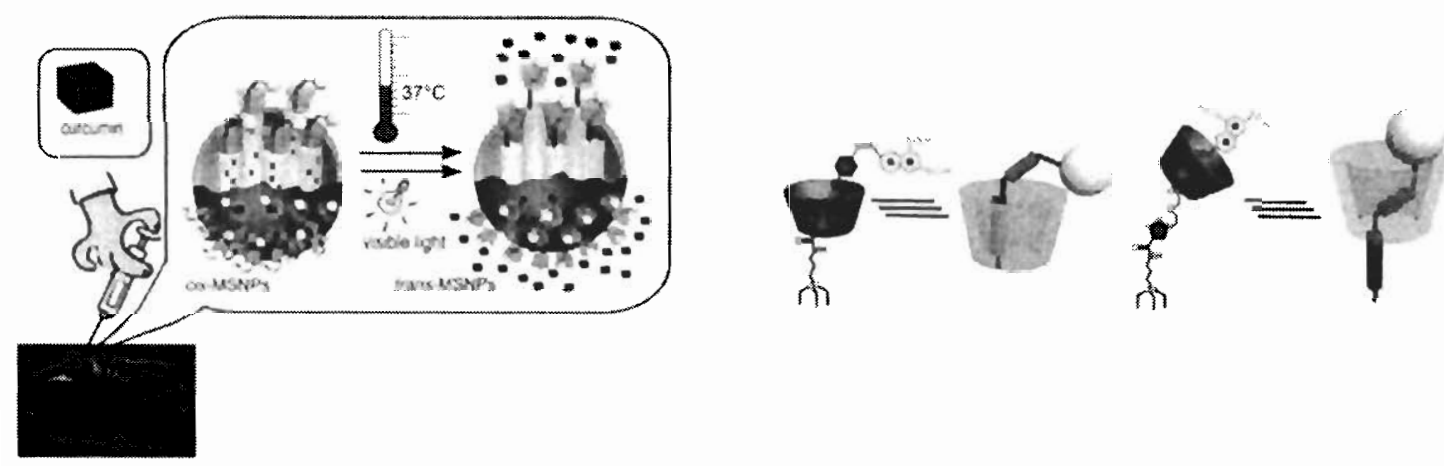

Figure 23 The graphical illustration of the thermal and photo triggered drug release after injection into the zebrafish, and the structure of the rotaxane. (Reproduced with permission from ref 172. Copyright 2012 Wiley.) 
Cyclodextrin polyrotaxanes produced by threading the CD rings onto a polymer chain following sealed by bulky end-groups, have drawn numerous attention because of its unique structures, since they are first described by Harada. In the past few decades, $C D$ polyrotaxanes have been widely investigated for biomedical applications such as drug delivery and bio imaging. ${ }^{40,136,161,173}$

Using CD as bulky end groups to make cyclodextrin polyrotaxanes as drug carrier, Jiang and co-workers developed a new strategy to prepare PTX grafted cyclodextrin polyrotaxane for cancer therapy. ${ }^{174}$ As shown in Figure 24, pseudopolyrotaxane was produced by mixing dialk-PEG with $\beta$-cyclodextrin, following sealed by the azide cyclodextrin via click reaction. Finally, the PTX was introduced by the succinate-based PTX ester coupling to afford the PTX grafted CD polyrotaxane. For in vivo experiments, the PTX grafted cyclodextrin polyrotaxane exhibited significant superior suppression ofsup the tumor growth and prolong circulation time than that of free Taxol. 


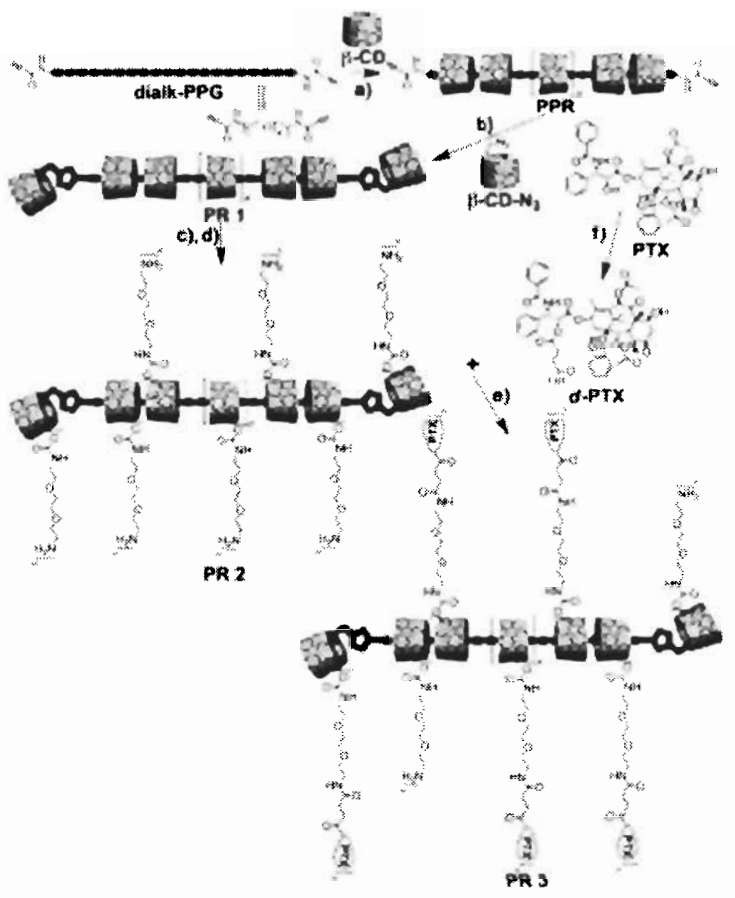

Figure 24 Schematic illustration of the synthesis route of the cylcodextrin polyrotaxane, and the PTX

bearing cyclodextrin polyrotaxane. (Reproduced with permission from ref 174. Copyright 2013 Wiley.)

\subsubsection{Supramolecular amphiphiles for biomedical applications based on Host-Guest}

\section{interactions}

Due to inherent hydrophilic property of the macrocyclic hosts, they will turn into supramolecular amphiphiles after binding with the hydrophobic guests. Compared with traditional amphiphiles, supramolecular amphiphiles show numerous advantages, because of its fabrication by non-covalent interactions. Supramolecular amphiphilies are constructed by two parts with various non-covalent interactions, which efficiently simplify the tedious covalent synthesis and functional modification of the traditional amphiphiles. Furthermore, the non-covalent and dynamic interactions allow the supramolecular amphiphiles to respond to various external stimulus. As the 
advantages mentioned above, supramolecular amphiphiles have garnered tremendous interest of the researchers, especially in biomedical relevant fields. ${ }^{52,148,153,175}$

Unlike naturally available CDs, CAs are artificially produced from phenols and formaldehyde. It comprises of phenolic units linked by methylene bridges at the meta-positions. Similar with CDs and CBs, there is a family of CA which includes C4A, C6A, and C8A. A slight differences from CDs and CBs is that odd numbers of CAs are hardly obtained by chemical synthesis. Compared with CDs and CBs, the CAs have weak affinity to guest molecules and poor water solubility. To solve these problems, many efforts have been made to prepare hydrophilic and water-soluble CAs. Sulfonation and carboxylation on the rims of the CAs are the common strategy to prepare water-soluble CAs and providing CAs strong affinity to positive charge guest molecules. ${ }^{176-180}$

An enzyme responsive supramolecular vesicle, constructed of p-sulfonatocalix[4]arene (SC4A) and Myristoylcholine, used to deliver tacrine for specifically Alzheimer's disease therapy was developed by Liu group. ${ }^{181}$ As show in Figure 25, under the treatment with $\mathrm{BChE}$, free Myristoylcholine formed micelle was not dissipated but transferred to another micelle, resulting in no drug release due to hydrophilic-hydrophobic balance maintained after the substrate shifted to product. Upon addition of SC4A, the supramolecular amphiphiles were obtained and self-assembled into a binary vesicle at concentration much lower than the CMC of the Myristoylcholine. The supramolecular vesicle can be dissipated to release tacrine via 
butyrylcholinesterase induced cleavage. This supramolecular amphiphilic system exhibits promising potential for delivery therapeutics to Alzheimer's disease therapy.

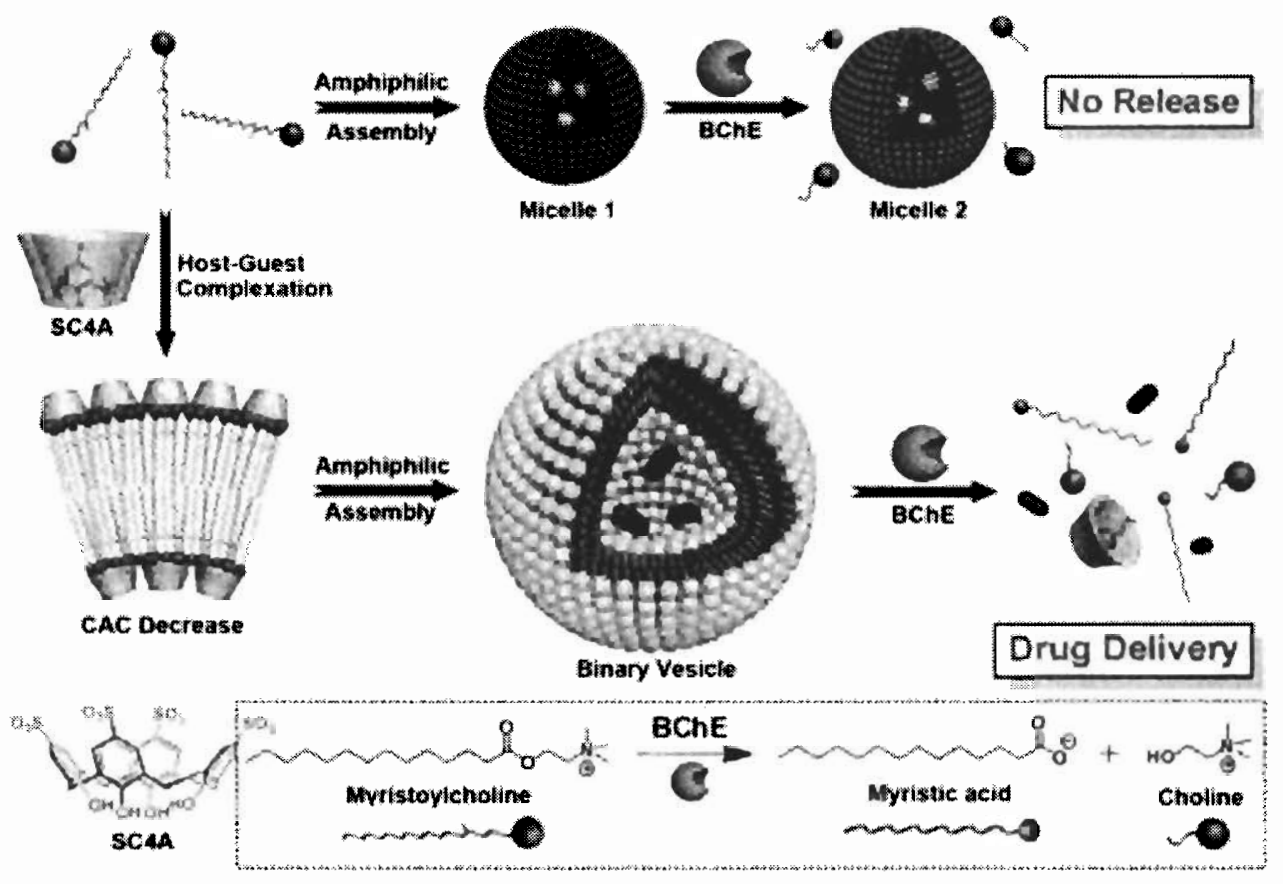

Figure 25 Schematic Illustration of Amphiphilic Assemblies of Myristoylcholine in the Absence and

Presence of SC4A, and enzyme triggered release mechanism. (Reproduced with permission from ref

\section{Copyright 2012 American Chemical Society.)}

Macrocyclic host molecules are also grafted on the polymers to provide the multivalent inclusion complex to obtain strong nano-assemblies. Using cyclodextrins grafted polymer as hydrophilic segment and PTX modified polymer as hydrophobic segment, Kim and co-workers fabricated a nano-assembly for cancer therapy. ${ }^{182}$ As shown in Figure 26, polymeric CD and polymeric PTX were obtained by reaction with polymeric maleic anhydride. The therapeutic platform shows both good water dispersibility and high stability in blood, which allows the nanoparticles to be efficiently uptake by cancer cells via EPR based passive targeting and receptor 
mediated active targeting mechanisms. After uptake by the tumor tissues, the ester linker between the polymer backbone and the CD and PTX will be cleaved by intracellular esterase to release the free PTX. This nano-platform exhibited significant tumor suppression in a mouse tumor model.
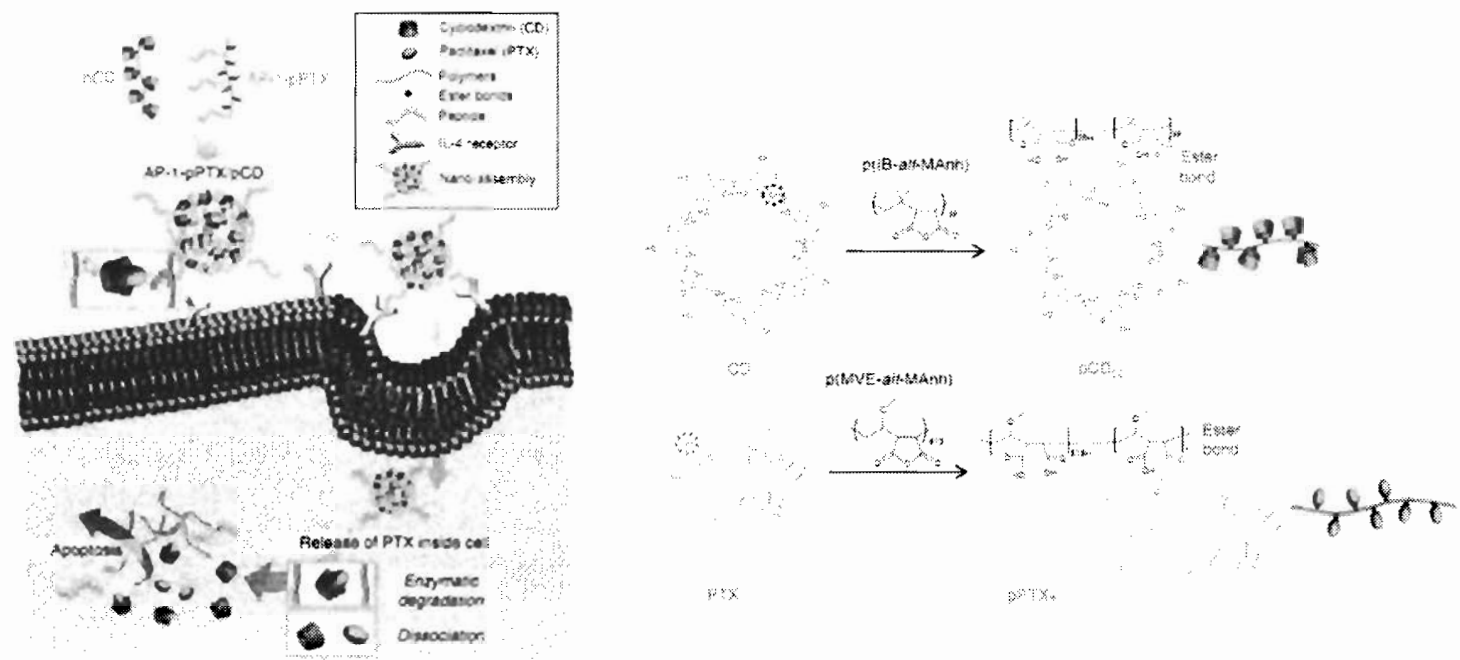

Figure 26 Schematic illustration of the self-assembly multivalent inclusion complexes between polymeric CD and polymeric PTX. The structure and synthesis route of the polymeric CD and polymeric PTX. (Reproduced with permission from ref 182. Copyright 2014 Nature

\subsubsection{Cyclodextrin-based gene delivery systems.}

Gene therapy has gained considerable attention in the past several decades, due to promising therapeutic application in genetic diseases, cancer, cardiovascular and infectious diseases. Because its rapid degradation by serum nucleases in the blood, much efforts have been made to develop effective carrier vectors. At first many attentions are focused on virus-related carriers which exhibit high efficiency at delivering gene to many types of cell lines. However, the potential toxicity and immunogenicity restrict the applications of such viral carriers. 
Numerous nonviral vectors for gene delivery have been developed, including polymers, dendrimers, cationic lipids, and nature macromolecules. Though nonviral vectors avoid the potential immune toxicity, they also face some drawbacks such as low transfection efficiency and cell toxicity. Development of novel non-viral vectors without the toxicity and high transfection efficiency for gene delivery has thus caught the attention of scientists. For the reason that cyclodextrin exhibits inherent high biocompatibility and could be easily modified, it is considered as an ideal platform to develop non-viral gene transfection vectors. ${ }^{22,39,183-186}$

In recent times, Li's group developed a multifunctional supramolecular gene delivery system based on supramolecular nanoparticles formed by host-guest interactions $^{187}$. As shown in Figure 27, cyclodextrin was modified with pDMAEMA (poly(2-dimethylaminoethyl methacrylate)) via disulfide linkage. Poly(2-methacryloyloxyethyl phosphorylcholine) (pMPC) was conjugated with adamantane as guest. The zwitterionic phosphorylcholine endowed the nanoparticles with excellent protein stability, high serum tolerance, cellular uptake and intracellular reduction responsive DNA release properties, and also low cytotoxicity. In in vitro experiments, nanoparticles exhibited very high transfection efficiency of the therapeutic gene p53 and showing promising potential for cancer gene therapy application. 
a) Formation of fCD-SS-PIAd-pMPC supramolecular DNA delivery system
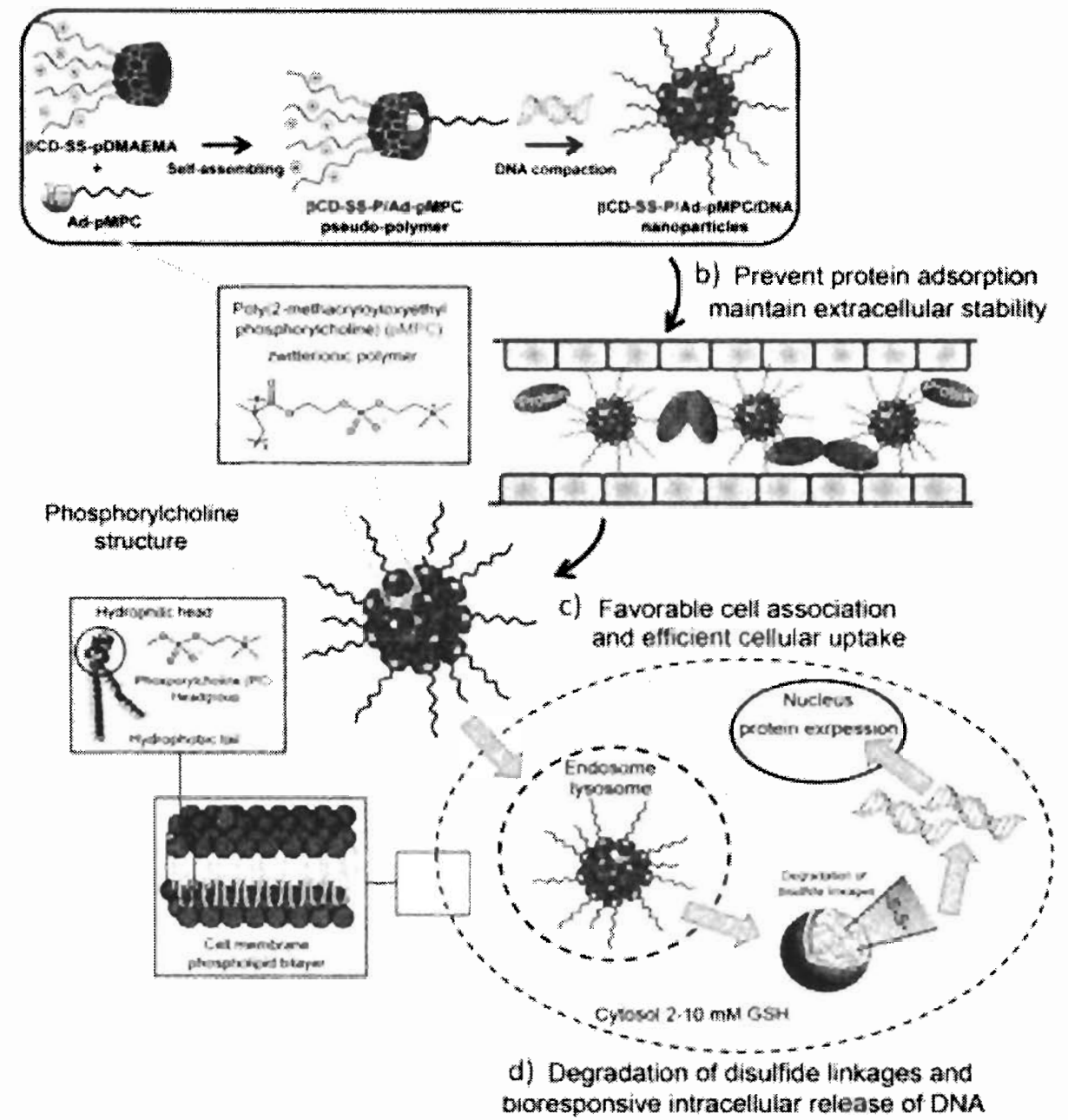

Figure 27 Conceptual illustration of $\beta$-CD-SS-PIAd-PMPC supramolecular gene delivery system. The structure of the cyclodextrin and adamantine guest and the intracellular gene transfection mechanism. (Reproduced with permission from ref 187. Copyright 2014 Wiley.)

Currently, small interfering RNA (siRNA) have drawn lots of attention as gene therapeutic agents. Conversely, as compared to DNA, siRNAs face much more serious degradation risks, due to rapid degradation by RNases and are excreted in the kidney. Thereby, more sophisticated vectors with low toxicity and high transfection efficiency are urgently needed for siRNA delivery. ${ }^{23,118,188-190}$ Using polymeric cyclodextrin as siRNA delivery vector, Davis group reported the first in-human phase 
I clinical trial concerning systemic administration of siRNA to patients bearing solid tumors. ${ }^{157,188}$ As shown in Figure 28, the nanoparticles were composed of four moieties, therapeutic siRNA and a linear polymeric cyclodextrin formed the nanoparticles with the cyclodextrin on the surface; adamantane modified PEG acted as the hydrophilic segment and provided the serum stability; and the adamantane PEG-TF (human transferrin protein) endowed the active targeting ability by TF receptor mediated endocytosis. The nanoparticles exhibited effective therapeutics performance in human body and dose-dependent accumulation in human tumors. This work is the first case that prove that RNAis in a human from an administered siRNA.

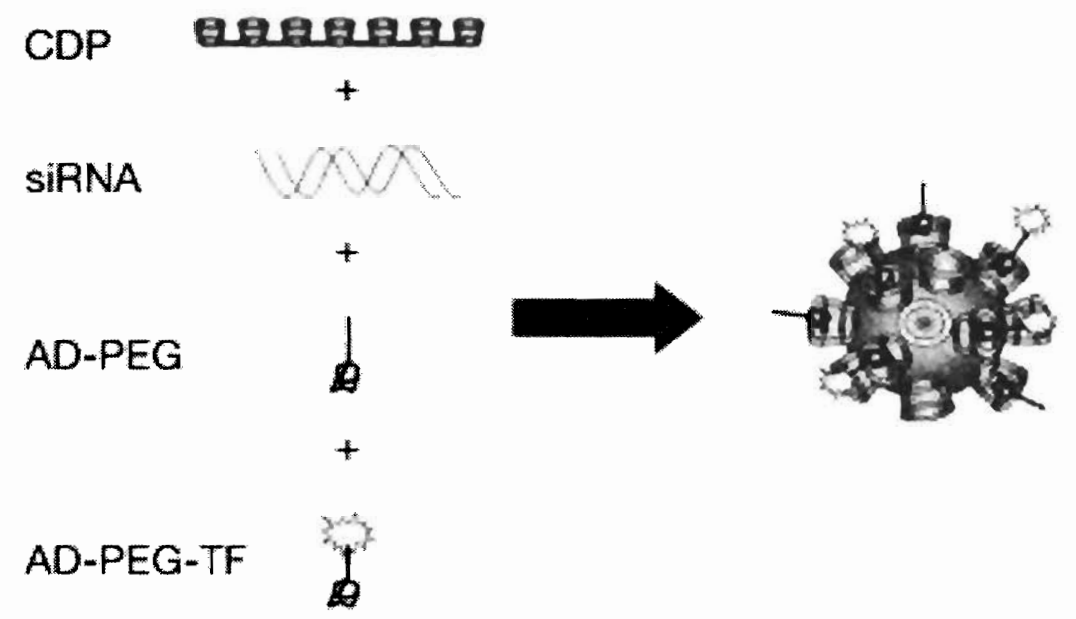

Figure 28 Schematic illustration of the polymeric cyclodextrin formed targeted nanoparticles, Ad-PEG for steric stabilization and PEG-TF for targeting. (Reproduced with permission from ref 188. Copyright 2010 Nature Publisting Group.) 


\section{Chapter 2 Prodrug Self-assembled Glutathione-responsive Vesicle for Targeted Chemotherapy in Combination with Intracellular Imaging}

\subsection{Introduction}

In the past decades, targeted drug delivery systems for cancer therapy have drawn considerable attention due to rapidly increasing requirements in accurate cancer therapeutics. To improve therapeutic efficiency and decrease side effects, numerous techniques have been developed to achieve better therapeutic performance. Among them, nano-carriers including liposomes, mesoporous silica nanoparticles, micelles, vesicles, polymer capsules, polymer dots, and organic/inorganic nanoparticles have been extensively applied to deliver therapeutic agents, since nanosized particles could preferentially accumulate in tumor tissues because of the enhanced permeability and retention (EPR) effect. By modifying targeting ligands such as RGD (Arg-Gly-Asp) peptides, folic acid, biotin, trans ferritin and sugar on the surface, these nano-carriers have exhibited high selective uptake efficiency with reduced side effects as compared with the free chemotherapeutic drugs. Although some nanomedicines have already exhibited better therapeutic efficacy than conventional anticancer drugs, a lot of problems still exist in these systems such as low drug loading content, premature leakage, insufficient drug release, and minimal renal clearance.

Another strategy is to construct a prodrug which can be converted into its active form after uptake by tumor tissues for the purpose of improving treatments. Prodrugs 
display many advantages over free drugs such as better water solubility, higher tumor selectivity, longer serum stability, and less side-effects. As chemotherapy drugs are difficult to be modified and purified, the most popular approach is to prepare polymeric prodrugs. Compared with free drugs, polymeric prodrugs display several advantages including easy preparation, controlled drug release, prolonged drug action, and remarkable EPR effect. However, polymeric prodrugs face one obvious drawback i.e. low drug content due to the inherent conflict between the hydrophilic segment and the hydrophobic segment. On the other hand, small molecule prodrugs have much higher drug content, but there are only a few reported works which directly used small molecule prodrugs to construct nanoparticles due to complicated synthesis of construction amphiphilic small molecule drugs. In addition, many prodrugs without stimuli-responsive units face very slow drug release under physiological conditions, so various cleavage linkers which can respond to acidic and reducing environments inside cancer cells, have been introduced into the prodrugs.

Typically, prodrugs with a cleavable unit can transform to its original active form inside cancer cells, but techniques to directly monitor this process are still lacking. In recent years, several groups reported some pioneering works focusing on this special field. By introducing fluorescence responsive groups into prodrugs, obvious fluorescence red shifts or recovery can be observed after uptake by cancer cells due to special microenvironments including low $\mathrm{pH}$, high $\mathrm{GSH}$ concentrations, overexpression enzymes, and high hydrogen peroxide concentrations. Fluorescence changes imply that linkers are cleaved and the prodrugs are activated, leading to 
monitoring of drug release process in real time. Besides monitoring the drug release process, this obvious fluorescence changes can also be utilized in intracellular imaging. To date, most of the theranostic prodrugs reported are used in directly and few of them are encapsulated inside of the nano carriers. So directly using prodrugs with a fluorescence reporter and targeting ligands to construct nanosized assemblies, has become a challenge yet a promising work in drug delivery systems.

Herein, we developed a small molecule prodrug (conjugate 1) self-assembled vesicle for targeted delivery of an anti-cancer drug to cancer cells. Conjugate 1 is composed of two parts: a water-soluble targeting group and a water-insoluble fluorescence reporter functionalized anticancer drug. D-mannose was a natural sugar which was employed as the hydrophilic part and tumor-targeting group because of mannose receptors overexpressed on lots of types of cancer cells ${ }^{188,189}$. D-Mannose was conjugated with naphthalimide through a triazole ring formation, and then naphthalimide was connected with chlorambucil anticancer drug via a disulfide bond. Naphthalimide was employed as a fluorescence responsive reporter whose emission underwent significantly red shifts from blue to green after disulfide bond cleavage by intracellular GSH. Chlorambucil, a commercial available alkylating agent, was used as a model drug, and works by sticking to one of the cancer cell's DNA strands ${ }^{193-198}$. Conjugate 1 can form nanosized vesicle structures with a mannose-rich surface by molecular self-assembly in aqueous solution, displaying improved cancer cell uptake efficiency by mannose receptor-mediated endocytosis and increase in potential tumor uptake via passive accumulated namely EPR effect. 
As shown in Scheme 1, conjugate 1 was synthesized by using 'click' reaction of 2'azidoethyl -O- $\alpha$-D-mannopyranoside with conjugate 2 . The compounds were fully characterized by using ${ }^{1} \mathrm{H}$ and ${ }^{13} \mathrm{C}$ NMR spectroscopy and high resolution mass spectroscopy (HRMS).

a)

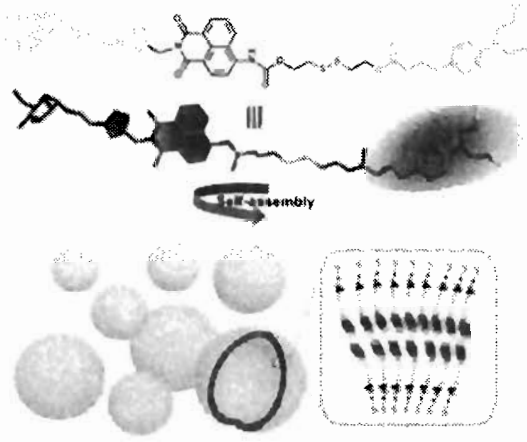

b)

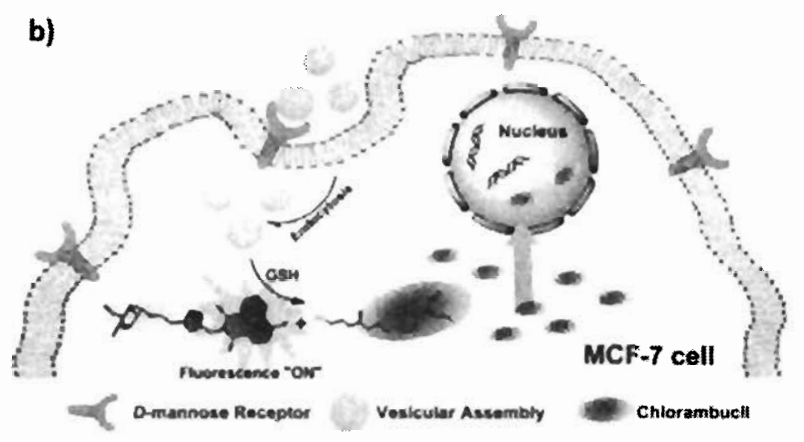

Scheme 1 a) The chemical structure of conjugate 1 and schematic illustration of the self-assembly of

conjugate 1 in aqueous solutions. b) Representation of the vesicle to be selectively uptake by MCF-7

cells by D-mannose receptor and dissipated by GSH, leading to fluorescence red-shifts and drug

delivery.

\subsection{Materials and Characterizations}

THF and toluene were refluxed with sodium overnight before used. Dichloromethane (DCM) and was refluxed with $\mathrm{CaH}_{2}$ overnight before used. $N^{\prime}, N^{\prime}$-dimethylformamide (DMF) was refluxed with $\mathrm{CaH}_{2}$ overnight and distilled under the reduced pressure before used. Ethanol was refluxed with magnesium and iodine overnight and distilled before used. $N, N$-diisopropylethylamine (DIPEA), 2,2‘-dithiodiethanol, 4-dimethylaminopyridine (DMAP), Chlorambucil, $N, N, N^{\prime}, N^{\prime \prime}, N^{\prime \prime}$-pentamethyldiethylenetriamine $\quad$ (PMDETA), sodium azide, 
Iodo(triethyl phosphite)copper(I), propargylamine, Tin(II) chloride dehydrate, 2-bromoethanol, 4-nitro-1,8-naphthalic anhydride, palladium on carbon, D-mannose, and triphosgene were obtained from Sigma Aldrich and were used without further purification. 1-Ethyl-3-(3-dimethylaminopropyl) carbodiimide (EDC) was purchased from $\mathrm{TCI}$ and used without further purification. $N$-Propargyl-4-amido-1,8-naphthalimide (compound 4) was synthesized according literature reported procedures. ${ }^{199}$

${ }^{1} \mathrm{H}$ and ${ }^{13} \mathrm{C}$ nuclear magnetic resonance (NMR) spectra were measured on a Bruker BBFO-400 spectrometer using deuterated chloroform $\left(\mathrm{CDCl}_{3}\right)$ and deuterated dimethyl sulphoxide (DMSO-d6) as the solvents. The electronic spray ionization (ESI) mass spectra were recorded on a ThermoFinnigan LCQ quadrupole ion trap mass spectrometer. High-resolution mass spectrometry (HR-MS) was performed on a Waters Q-tof Premier MS spectrometer. Transmission electron microscopy (TEM) images were collected on JEM-1400 (JEOL) at $100 \mathrm{kV}$. Scanning electron microscope (SEM) images was collected on from a SEM of field-emission JSM-6700F (JEOL, Japan) operated at $100 \mathrm{kV}$. The fluorescence emission spectra were recorded on a Shimadzu RF-5301pc fluorescence spectrophotometer. UV-vis spectra were recorded from Shimadzu UV-3600 spectrophotometer. Zeta-potential values were determined by a Malvern Instruments Zetasizer Nano-S at $25^{\circ} \mathrm{C}$. DLS size distributions were measured on a Nanobrook 90Plus particle size analyzer. MTT was recorded on a Tecan Infinite M200. Flow cytometry was recorded on a BD LSR Fortessa X20. 


\subsubsection{Synthesis of the compounds}

\subsubsection{Preparation of N-Propargy/4-nitro-1,8-naphthalimide}

Propargylamine $(0.4 \mathrm{~mL}, 6.16 \mathrm{mmol})$ was quickly added to a cloudy solution of 4-nitro-1,8-naphthalic anhydride $(1.36 \mathrm{~g}, 5.6 \mathrm{mmol})$ in absolute ethanol $(20 \mathrm{~mL})$. After $6 \mathrm{~h}$ of refluxing under $\mathrm{N}_{2}$, the reaction was allowed to cool to room temperature and filtered off, washed with cold ethanol $(10 \mathrm{~mL})$. The residue was dried in vacuo to afford the product ( $90 \%$ yield).

${ }^{\prime} \mathrm{H} \mathrm{NMR}\left(\mathrm{CDCl}_{3}, 400 \mathrm{MHz}\right): \delta 8.85(\mathrm{~d}, \mathrm{~J}=8.8 \mathrm{~Hz}, 1 \mathrm{H}), 8.78(\mathrm{~d}, 1 \mathrm{H}), 8.74(\mathrm{~d}, 1 \mathrm{H})$, $8.41(\mathrm{~d}, 1 \mathrm{H}), 8.01(\mathrm{dd}, 1.2 \mathrm{~Hz}, 1 \mathrm{H}), 4.97(\mathrm{~d}, 2 \mathrm{H}), 2.23(\mathrm{t}, 1 \mathrm{H}) \mathrm{ppm} ;{ }^{13} \mathrm{C} \mathrm{NMR}\left(\mathrm{CDCl}_{3}\right.$, $100 \mathrm{MHz}$ ): $\delta 162.8,162.7,149.7,132.5,130.2,130.0,129.7,129.1,126.6,123.8$, 123.7, 122.7, 77.8, 71.1, 29.8 ppm; ESI-MS: (m/z) $281.1[\mathrm{M}+\mathrm{H}]+$, calcd. for $\mathrm{C}_{15} \mathrm{H}_{9} \mathrm{~N}_{2} \mathrm{O}_{4}=281.1$

\subsubsection{Preparaiton of N-Propargy/4-amido-1,8-naphthalimide}

To a stirred cloudy solution of N-Propargyl-4-nitro-1,8-naphthalimide (420 mg, 1.5 mmol) in absolute ethanol $(12 \mathrm{~mL})$ was added dropwise the solution of Tin(II) chloride dehydrate $(2.3 \mathrm{~g}, 9 \mathrm{mmol})$ in concentrated hydrochloric acid $(3 \mathrm{~mL})$ at room temperature. The reaction was quenched with aqueous $10 \% \mathrm{Na}_{2} \mathrm{CO}_{3}$ and filtered off. After washed with water $(3 \times 20 \mathrm{~mL})$ the residue was dried in vacuo to afford compound 1 ( $85 \%$ yield).

${ }^{\prime}$ H NMR (DMSO-d $\left.6,400 M H z\right): \delta 8.62(\mathrm{~d}, 1 \mathrm{H}), 8.43(\mathrm{~d}, 1 \mathrm{H}), 8.19(\mathrm{~d}, 1 \mathrm{H}), 7.65(\mathrm{t}$, $1 \mathrm{H}), 6.85(\mathrm{~d}, 1 \mathrm{H}), 4.72(\mathrm{~d}, 2 \mathrm{H}), 3.05(\mathrm{t}, 1 \mathrm{H}) \mathrm{ppm} ;{ }^{13} \mathrm{C}$ NMR (DMSO-d $\left.\mathrm{d}_{6}, 100 \mathrm{MHz}\right): \delta$ 
163.0, 162.0, 153.0, 134.2, 131.2, 129.7 (2C), 124.0, 121.4, 119.3, 108.2, 107.0, 80.0, 72.3, 28.6 ppm; ESI-MS: (m/z) $250.8[\mathrm{M}+\mathrm{H}]+$, calcd. for $\mathrm{C}_{15} \mathrm{H}_{11} \mathrm{~N}_{2} \mathrm{O}_{2}=251.1$.

\subsubsection{Preparation of compound 3}

To a mixture of compound $4(500 \mathrm{mg}, 2 \mathrm{mmol})$ and N,N-diisopropylethylamine (DIPEA, $878.8 \mathrm{mg}, 6.8 \mathrm{mmol})$ in anhydrous toluene $(30 \mathrm{~mL})$ was dropwise added a solution of triphosgene $(611.8 \mathrm{mg}, 2 \mathrm{mmol})$ in toluene $(8 \mathrm{~mL})$. The mixture was refluxed for $3 \mathrm{~h}$, and then allowed to cool down to room temperature. The solvent was removed under the reduced pressure. A solution of 2,2'-dithiodiethanol in anhydrous THF (30 mL) was added to the flask. The reaction mixture was stirred overnight under room temperature. The solvent was evaporated off, at which point $\mathrm{CH}_{2} \mathrm{Cl}_{2}(100$ $\mathrm{mL})$ and water $(100 \mathrm{~mL})$ were added, and the organic layer was collected. The $\mathrm{CH}_{2} \mathrm{Cl}_{2}$ layer was dried using anhydrous $\mathrm{NaSO}_{4}$. After the removal of the solvent, the crude product was purified over silica gel using ethyl hexane/acetate $(\mathrm{v} / \mathrm{v}, 3: 1)$ as the eluent to remove the impurity and then hexane/acetate $(\mathrm{v} / \mathrm{v}, 1: 1)$ as the eluent to yield compound 3 as a light yellow solid (550.0 mg, 64\%).

${ }^{\prime} \mathrm{H}$ NMR (400 MHz, $\left.\mathrm{CDCl}_{3}, 298 \mathrm{~K}\right): \delta 8.65(\mathrm{~m}, 2 \mathrm{H}), 8.39(\mathrm{~d}, 1 \mathrm{H}), 8.27(\mathrm{~d}, 1 \mathrm{H})$ $7.79(\mathrm{~m}, 2 \mathrm{H}), 4.94(\mathrm{~d}, 2 \mathrm{H}), 4.57(\mathrm{t}, 2 \mathrm{H}), 3.93(\mathrm{~m}, 4 \mathrm{H}), 3.07(\mathrm{t}, 2 \mathrm{H}), 2.94(\mathrm{~m}, 2 \mathrm{H}), 2.89$ (t, 2H), $2.19(\mathrm{t}, 1 \mathrm{H}) .{ }^{13} \mathrm{C}$ NMR $\left(100 \mathrm{MHz}, \mathrm{CDCl}_{3}, 298 \mathrm{~K}\right): \delta 163.4,162.8,152.9$, $139.3,133.0,131.7,129.0,126.7,126.5,123.1,116.9,78.5,70.5,63.9,60.6,60.4$, 41.6, 37.5, 29.7, 29.4. HRMS (TOF) $\mathrm{m} / \mathrm{z}[\mathrm{M}+\mathrm{H}]^{+}$, calcd for $\mathrm{C}_{20} \mathrm{H}_{18} \mathrm{~N}_{2} \mathrm{O}_{5} \mathrm{~S}_{2}, 431.0735$; found, 431.0735 . 


\subsubsection{Preparation of conjugate 2}

Chlorambucil (350 mg, $1.16 \mathrm{mmol}$ ), 4-dimethylaminopyridine (DMAP, $100 \mathrm{mg}$ $1.1 \mathrm{mmol}$ ) and 1-ethyl-3-(3-dimethylaminopropyl) carbodiimide (EDC, $300 \mathrm{mg}, 1.3$ mmol) were dissolved in dried DCM $(10 \mathrm{~mL})$ and the mixture was stirred at $0{ }^{\circ} \mathrm{C}$ for 1 h. A solution of compound $3(100 \mathrm{mg}, 0.232 \mathrm{mmol})$ in dried DCM $(10 \mathrm{~mL})$ was added to the mixture followed by DMAP $(100 \mathrm{mg}, 1.1 \mathrm{mmol})$. The mixture was allowed to stir at room temperature in the dark for $48 \mathrm{~h}$. The mixture was filtered to remove white solid. The filtrate was washed with water $(50 \mathrm{~mL})$ for 3 times, dried with anhydrous $\mathrm{Na}_{2} \mathrm{SO}_{4}$. The solvent was removed under the reduced pressure to give crude product. The crude product was purified by column silica gel chromatography using $\mathrm{DCM} / \mathrm{MeOH}(\mathrm{v} / \mathrm{v}, 500: 1)$ to afford the pure product as light yellow solid (120 $\mathrm{mg}, 72 \%)$.

${ }^{\mathrm{l}} \mathrm{H}$ NMR (400 MHz, $\left.\mathrm{CDCl}_{3}, 298 \mathrm{~K}\right): \delta 8.65(\mathrm{~m}, 2 \mathrm{H}), 8.39(\mathrm{~m}, 2 \mathrm{H}), 7.97(\mathrm{~s}, 1 \mathrm{H})$, $7.74(\mathrm{t}, 1 \mathrm{H}), 6.99(\mathrm{~d}, 2 \mathrm{H}), 6.59(\mathrm{~d}, 2 \mathrm{H}), 4.99(\mathrm{t}, 2 \mathrm{H}), 4.55(\mathrm{t}, 2 \mathrm{H}), 4.42(\mathrm{t}, 2 \mathrm{H}), 3.69(\mathrm{~m}$, $2 \mathrm{H}), 3.61(\mathrm{~m}, 2 \mathrm{H}), 3.05(\mathrm{t}, 2 \mathrm{H}), 2.97(\mathrm{t}, 2 \mathrm{H}), 2.52(\mathrm{t}, 2 \mathrm{H}), 2.35(\mathrm{t}, 2 \mathrm{H}), 2.19(\mathrm{t}, 1 \mathrm{H})$, $1.87(\mathrm{~m}, 2 \mathrm{H}) .{ }^{13} \mathrm{C}$ NMR $\left(100 \mathrm{MHz}, \mathrm{CDCl}_{3}, 298 \mathrm{~K}\right): \delta 173.4,163.3,162.6,152.4$ $143.7,139.2,132.7,131.4,130.5,129.5,128.8,126.9,126.3,123.1,122.7,117.3$, $117.0,112.2,78.6,70.6,63.0,62.4,53.7,40.4,37.6,36.5,33.9,33.4,29.5,26.7$. HRMS (TOF) $\mathrm{m} / \mathrm{z}[\mathrm{M}+\mathrm{H}]^{+}$, calcd for $\mathrm{C}_{20} \mathrm{H}_{18} \mathrm{~N}_{2} \mathrm{O}_{5} \mathrm{~S}_{2}, 716.1423$; found, 716.1420.

\subsubsection{Preparation of conjugate 1}

Conjugate $2(70 \mathrm{mg}, 0.98 \mathrm{mmol})$ and 2'-azidoethyl-O- $\alpha$-D-mannopyranoside (100 $\mathrm{mg}, 4 \mathrm{mmol})$ were dissolved in anhydrous DMF $(10 \mathrm{~mL}) . \mathrm{CuBr}(15 \mathrm{mg}, 0.01 \mathrm{mmol})$ 
was added to the solution under the nitrogen atmosphere. Then, $N, N, N$, $N$ ", $N$ ''-pentamethyldiethylenetriamine (PMDETA, $18 \mathrm{mg}, 0.01 \mathrm{mmol}$ ) was injected into the solution by syringe. The mixture was allowed to stir at $50{ }^{\circ} \mathrm{C}$ under the nitrogen S-4 protection for $48 \mathrm{~h}$. The solvent was removed under the reduced pressure to give yellow wax. $\mathrm{MeOH}(20 \mathrm{~mL})$ was added to dissolve the solid, and silica gel $(1 \mathrm{~g})$ was added to the mixture. After the removal of the solvent, the mixture was purified by column silica gel chromatography. First, ethyl acetate/MeOH (v/v, 38:1) was used as eluent to remove the impurity. Then, DCM/MeOH (v/v, 9:1) was used as eluent to afford the product as light yellow wax (49 mg, 53\%).

${ }^{1} \mathrm{H}_{\mathrm{NMR}}\left(400 \mathrm{MHz}, \mathrm{DMSO}_{6}, 298 \mathrm{~K}\right): \delta 10.40(\mathrm{~s}, 1 \mathrm{H}) 8.73(\mathrm{~d}, 1 \mathrm{H}), 8.53(\mathrm{~m}$, $2 \mathrm{H}), 8.19(\mathrm{~d}, 1 \mathrm{H}) 8.01(\mathrm{~s}, 1 \mathrm{H}), 7.86(\mathrm{~m}, 2 \mathrm{H}), 6.99(\mathrm{~d}, 2 \mathrm{H}), 6.64(\mathrm{~d}, 2 \mathrm{H}), 5.30(\mathrm{~s}, 2 \mathrm{H})$, 4.2-4.77 (m, 17H), $3.91(\mathrm{~m}, 2 \mathrm{H}), 3.74-3.82(\mathrm{~m}, 2 \mathrm{H}), 3.39-3.54(\mathrm{~m}, 7 \mathrm{H}), 3.11(\mathrm{t}, 2 \mathrm{H})$, $3.03(\mathrm{t}, 2 \mathrm{H}), 2.42(\mathrm{t}, 2 \mathrm{H}), 2.28(\mathrm{t}, 2 \mathrm{H}), 1.75(\mathrm{~m}, 2 \mathrm{H}) .{ }^{13} \mathrm{C} \mathrm{NMR}\left(100 \mathrm{MHz}, \mathrm{CDCl}_{3}, 298\right.$ $\mathrm{K}): \delta 173.1,163.9,162.9,154.3,145.1,141.5,132.2,131.7,130.1,129.8,129.1$, $126.9,124.6,124.1,122.6,120.1,119.0,117.8,112.4,100.1,78.3,74.6,74.2,71.3$, $70.7,70.5,67.6,67.4,67.3,66.1,65.2,52.7,50.5,50.4,48.7,41.6,37.1,36.9,33.7$, 33.3, 29.3, 29.0. HRMS (TOF) $\mathrm{m} / \mathrm{z}[\mathrm{M}+\mathrm{H}]^{+}$, calcd for $\mathrm{C}_{20} \mathrm{H}_{18} \mathrm{~N}_{2} \mathrm{O}_{5} \mathrm{~S}_{2}, 965.2383$; found, 965.2382 .

\subsubsection{Preparation of vesicles}

Conjugated 1 was dissolved in DMSO to prepare stock solution of concentration 4 $\mathrm{mM}$. Under the sonication condition, the $10 \mu \mathrm{L}$ stock solution was slowly added into the DI water. The obtained solution was sonicated for another 30 mins, followed by 
stabilization at room temperature overnight and the solution was dialysed against water for $2 \mathrm{~h}$. The cell incubation medium was also used to prepare vesicle by changing the water to Dulbecco's modified eagle medium (DMEM).

\subsubsection{Drug release mechanism}

The GSH triggered release behavior was studied by using LC-MS and HPLC. The vesicle solution was prepared via using PBS buffer with $\mathrm{pH} 7.4$, followed by adding GSH. The mixture was incubated at $37{ }^{\circ} \mathrm{C}$ for $4 \mathrm{~h}$, and the obtained solution was characterized with LC-MS and HPLC. Acetonitrile and water were used as eluents of the HPLC, and the ratio of the two kinds of the solvents was changed along the time. The ratio of the acetonitrile to water was 95 to 5 at 0-25 $\mathrm{min}$, and 5 to 95 at 25-32 $\mathrm{min}$, finally 95 to 5 till $40 \mathrm{~min}$. The monitor absorption wavelength was $270 \mathrm{~nm}$.

\subsubsection{Cellular uptake study}

HeLa cells and MCF-7 cells were cultured with DMEM with $10 \%$ fetal bovine serum (FBS), 1\% penicillin and streptomycin (PS) under 5\% $\mathrm{CO}_{2}$ atmosphere at $37^{\circ} \mathrm{C}$.

For confocal studies, HeLa cells and MCF-7 cells were seeded in a 6-well tissue culture plate $(2 \mathrm{~mL}$ medium $)$ with a density of $2.0 \times 10^{5}$ cells per well on the slides. After culturing $24 \mathrm{~h}$, the vesicle was added into the culture medium with the concentration of $10 \mu \mathrm{M}$. After incubation with vesicles at different times, the culture medium was removed, cells were washed with the PBS 3 times and then fixed with 4\% formaldehyde at room temperature for $15 \mathrm{~min}$. 
Flow cytometry was also utilized to investigate the cellular uptake. HeLa cells and MCF-7 cells were also seeded in a 6 -well tissue culture plate $(2 \mathrm{~mL}$ medium $)$ at a density of $2.0 \times 10^{5}$ cells per well. Conjugate 1 and conjugate 2 were added into the medium and incubated for $12 \mathrm{~h}$, cells were washed with PBS 3 times, and then treated with trypsin $(0.5 \mathrm{~mL})$. Subsequently, fresh medium $(0.5 \mathrm{~mL})$ was added to each culture well, and the cells were collected via centrifugation at $2500 \mathrm{rpm}$ for $2 \mathrm{~min}$. After washing with PBS (1 mL) for 2 times, the cells were suspended in PBS (1 mL) and subjected to the flow cytometry analysis.

\subsubsection{Cytotoxicity study}

MTT assay was used to investigate the cytotoxicity of conjugate 1 and free drug Chlorambucil. The HeLa cells and MCF-7 cells were seeded in 96-well plates $(100 \mu \mathrm{L}$ medium) and incubated for $24 \mathrm{~h}$. After the cell density reached $60 \%-70 \%$, the cells were fed with the conjugate 1 and Chlorambucil and incubated with $24 \mathrm{~h}$ and $48 \mathrm{~h}$, respectively. After the medium removed, the fresh medium with $10 \%$ MTT was added, and incubated with for another $4 \mathrm{~h}$. The medium was removed carefully, following with adding $100 \mu \mathrm{L}$ DMSO. Finally, optical densities of the samples were measured using a microplate reader with the double wavelength of $570 \mathrm{~nm}$ and $490 \mathrm{~nm}$.

\subsection{Results and discussion}

\subsubsection{Design and synthesis of the amphiphilic small molecule prodrug}

The synthesis route of the conjugate 1 was shown in scheme 2 . The disulfide linker was introduced by carboxamide bond between $2,2^{\prime}$-dithiodiethanol and compound 4 via triphosgene mediated reaction. Using EDC as catalyst, Chlorambucil was 
introduced by ester condensation reaction. Finally, the mannose was linked to naphthalimide via $\mathrm{Cu}$ (I) mediated azide-alkyne cycloadditions.

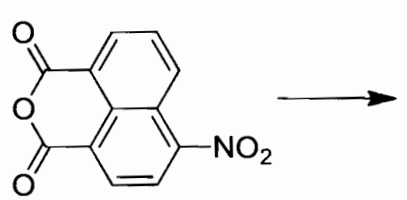

6

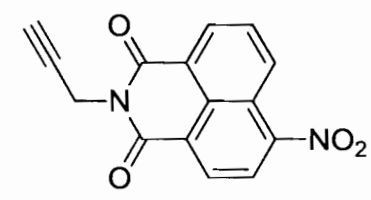

5

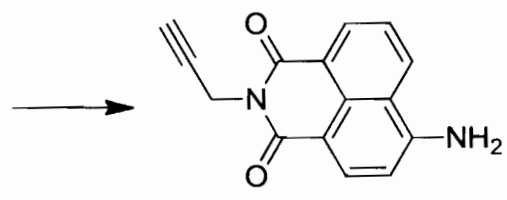

4<smiles>C#CCN1C(=O)c2cccc3c(NC(=O)OCCSSCCO)ccc(c23)C1=O</smiles><smiles></smiles><smiles>O=C(CCCc1ccc(N(CCCl)CCCl)cc1)OCCSSCCOC(=O)Nc1ccc2c3c(cccc13)C(=O)N(Cc1cn(CCOC(CO)C(O)CO)nn1)C2=O</smiles>

Conjugate 1

Scheme 2 The synthesis of the conjugate 1

\subsubsection{Self-assembly of the amphiphilic small molecule prodrug}

The inherent amphiphilic property of the conjugate 1 allows it to form vesicles in water. The critical aggregation concentration (CAC) of conjugate 1 was determined by monitoring the dependence of the optical transmittance at $650 \mathrm{~nm}$ on the increasing concentration of conjugate 1 ranging from $5.0 \mu \mathrm{M}$ to $50 \mu \mathrm{M}$. The optical 
transmittance decreased gradually upon increasing concentrations of conjugate 1 and a great decrease was observed at the concentration of $25 \mu \mathrm{M}$ due to formation of large aggregates, Figure 29. The solution of conjugate 1 exhibited obvious Tyndall effect, indicating that conjugate 1 successfully formed abundant nanoparticles. DLS was utilized to determine the hydrodynamic diameter $(\mathrm{DH})$ of the nanoparticle in aqueous solution. As shown in Figure 30 , conjugate 1 aggregates exhibit an average $D_{H}$ of 120 nm with a narrow distribution. From the transmission electron microscopy (TEM) images in Figure 30, the aggregates showed a uniform vesicle-like morphology with a diameter of about $110 \mathrm{~nm}$. Scanning electron microscopy (SEM) was also employed to characterize the vesicle, which also confirmed the aggregates had spherical morphology Figure 30.
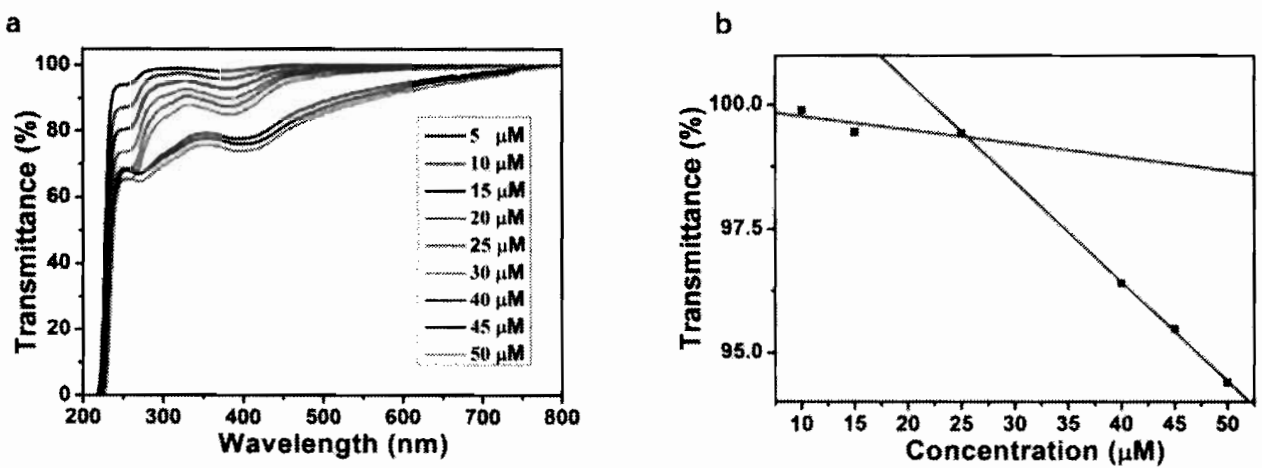

Figure 29 (a) Dependence of the optical transmittance at $650 \mathrm{~nm}$ on the conjugate 1 concentration (5-50 $\mu M$ ) in water at $25^{\circ} \mathrm{C}$. (b) Optical transmittance of conjugate 1 by increasing the concentration from 5 to $50 \mu M$ at $25^{\circ} \mathrm{C}$ in water. 
a

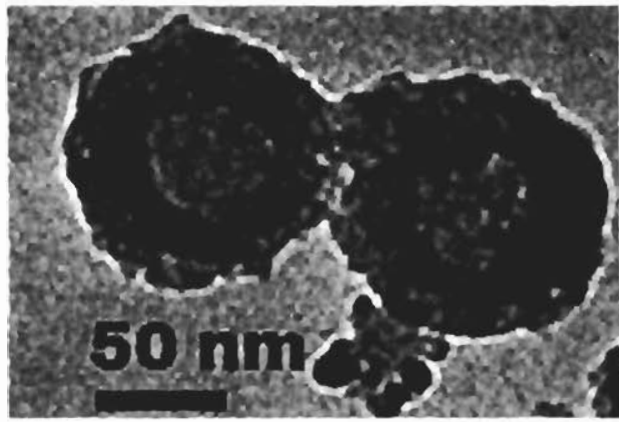

c

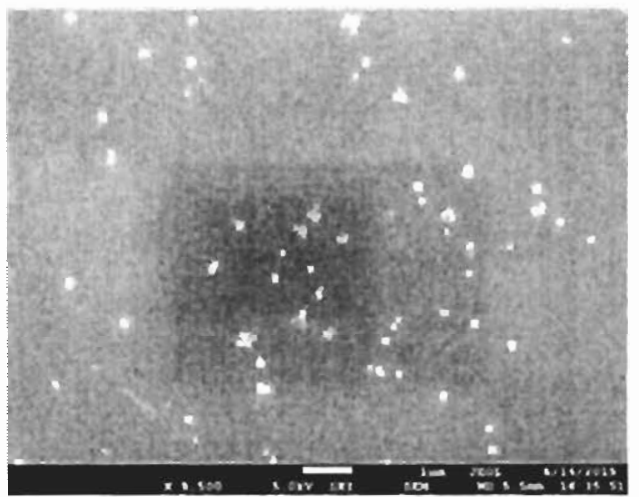

b

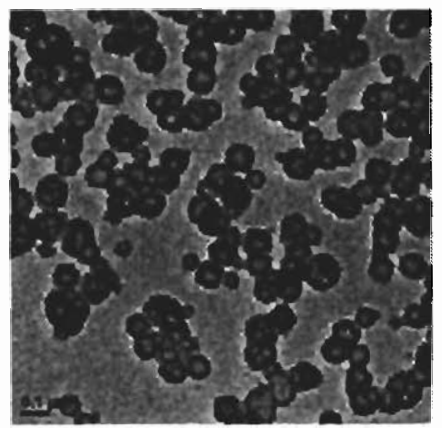

d

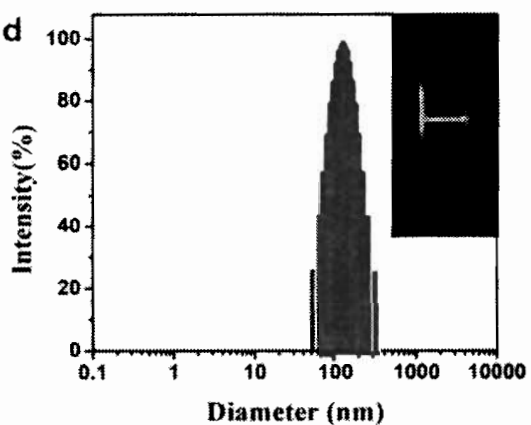

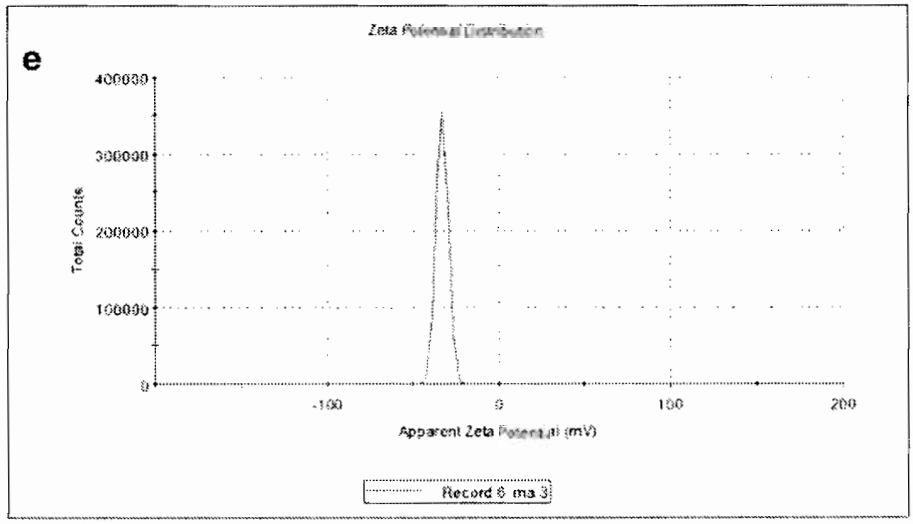

Figure 30 (a) and (b) TEM images of vesicular nanoparticles negatively stained with phosphomolybdic acid. Scale bar $=50 \mathrm{~nm}$. (c) SEM image of the vesicles. Scale bar $=1 \mu \mathrm{m} . \quad$ (d) $D L S$ data of the vesicles in water. Inset: a photo showing the Tyndall effect of the vesicle solution. (e) Zeta potential of the vesicle solution.

The stability of the vesicles in cell culture medium was also investigated. Similar DLS results and Tyndall effect of the vesicles in Dulbecco's modified eagle medium (DMEM) containing 10\% fetal bovine serum (FBS) and 1\% Pen-Strep were observed (Figure 31) with a narrow size about $110 \mathrm{~nm}$, indicating the suitability of the vesicles 
for cell studies. A negative surface charge of $-32.9 \mathrm{mV}$ was determined by Zeta potential (Figure 31), because the surface of the vesicles was full of negatively charged sugar units.
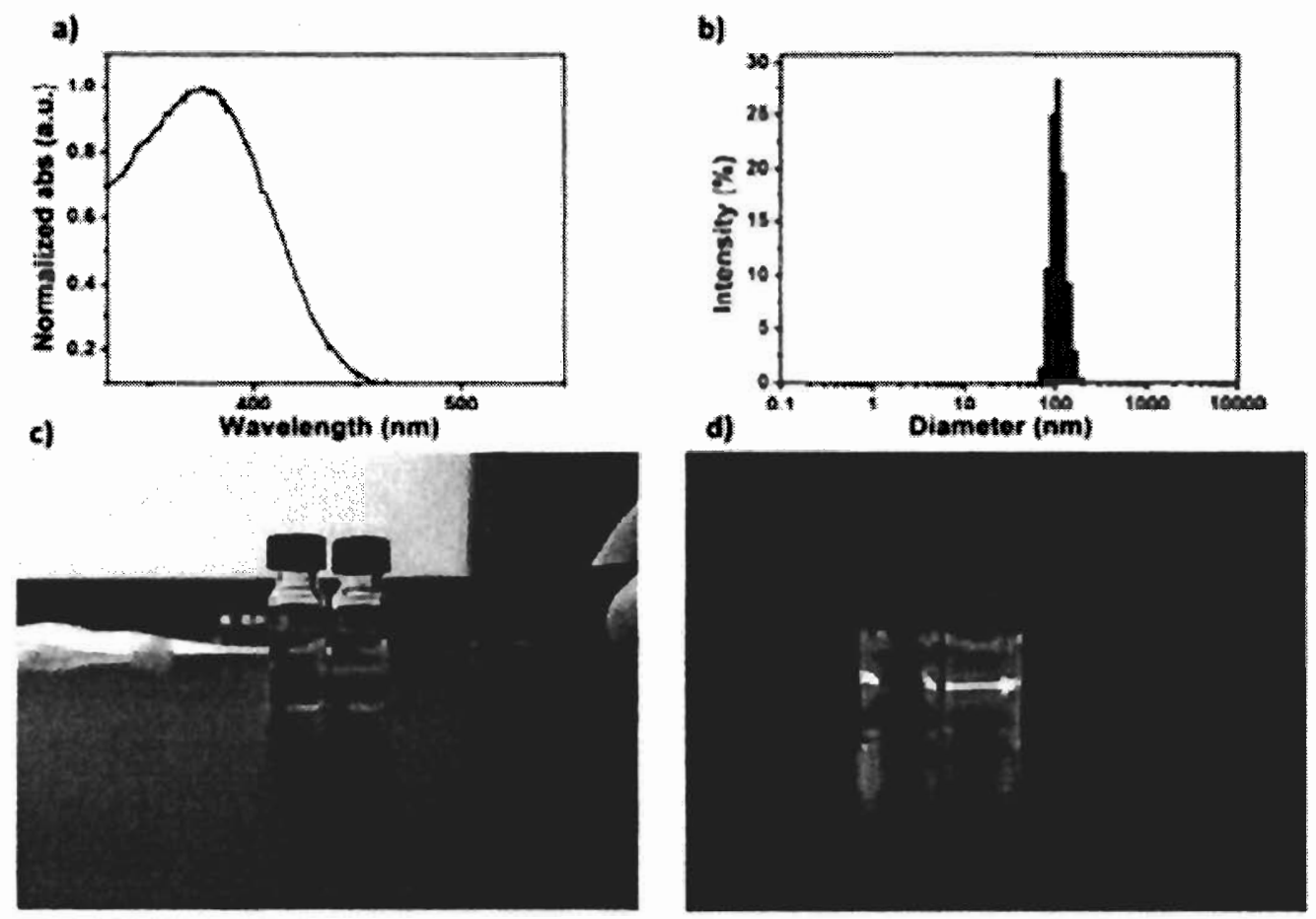

Figure 31 (a) UV-Vis spectrum of conjugate 1 under the treatment of oxidized GSH at $37^{\circ} \mathrm{C}$ in PBS (pH

7.4); (b) DLS data of vesicles in DMEM (including 10\% FBS and $1 \%$ Pen-Strep), (c. d) Tyndall effect of vesicles in DMEM (including 10\% FBS and $1 \%$ Pen-Strep).

\subsubsection{GSH triggered drug release behavior}

After incubating with GSH for $2 \mathrm{~h}$ at $37^{\circ} \mathrm{C}$ under physiological conditions, the solution was characterized by DLS and TEM (Figure 32). From the DLS data, a particularly wide size distribution of nanoparticles was observed from $300 \mathrm{~nm}-1300$ $\mathrm{nm}$, indicating the nanoparticles degraded to amorphous structures. The amorphous structures were also confirmed by TEM. The vesicles were degraded by introducing 
GSH into the solution as the aggregates lost the balance between hydrophilic and hydrophobic segments after disulfide bond cleavage.

Upon addition of GSH into the solution under physiological conditions, UV-vis and fluorescence spectra were utilized to monitor the spectroscopic changes (Figure 32). Before addition of GSH, the vesicles solution displayed a major absorption band from $350 \mathrm{~nm}$ to $500 \mathrm{~nm}$ centered at $380 \mathrm{~nm}$. When $\mathrm{GSH}(5.0 \mathrm{mM})$ was added into the solution, the maximum absorption peak exhibited an obvious red shift from $380 \mathrm{~nm}$ to $425 \mathrm{~nm}$ with the colour of the solution changing from colourless to light yellow (Figure 32 insert photography). In the fluorescence emission spectrum, the fluorescence change of the solution was monitored with time in the presence of GSH $(5.0 \mathrm{mM})$. The solution also displayed a rapid emission enhanced at $540 \mathrm{~nm}$, while a gradually decreasing emission was observed at $482 \mathrm{~nm}$ at the same time and accompanied with a colour change from blue to green. This responsive mechanism has been widely investigated in reported literature. At first, the disulfide bond is cleaved in the presence of GSH to form an unstable intermediate and an active drug. After which, the intermediate product undergoes an intramolecular cyclization to give the D-mannose/naphthalimide unit with the fluorescence changes (Scheme 3). Mass spectrometry and high-performance liquid chromatography (HPLC) studies confirmed the cleavage mechanism of conjugate 1 after the treatment of GSH. 
a
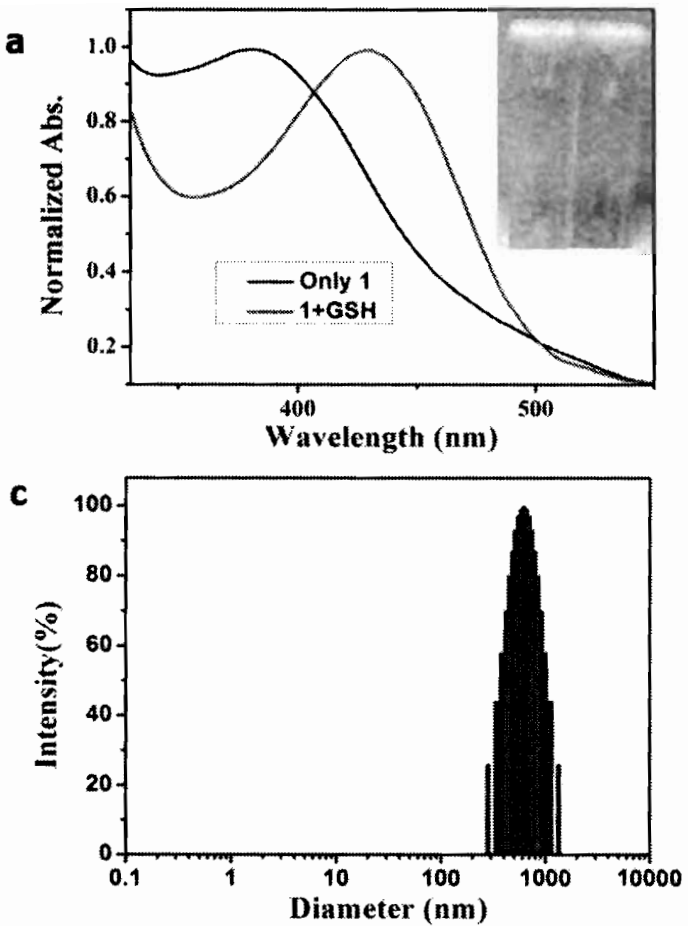

b

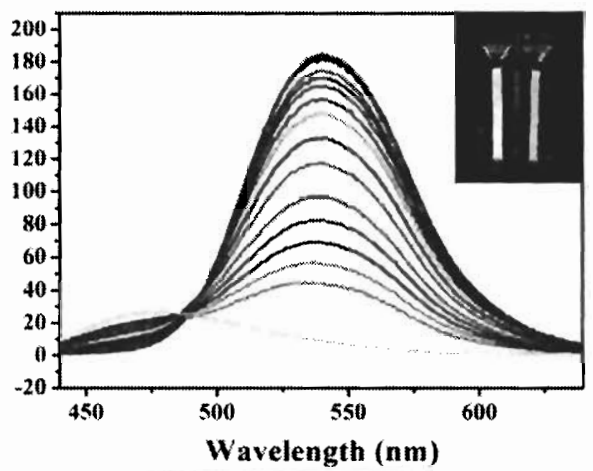

d

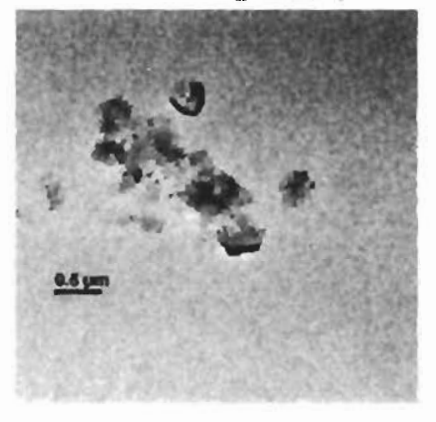

Figure 32 . (a) Absorption spectra of conjugate $1(10.0 \mu \mathrm{M})$ in the presence and absence of GSH $(5.0 \mathrm{mM})$ at $37^{\circ} \mathrm{C}$ in PBS buffer (pH 7.4). (b) Time-dependent fluorescence spectral changes observed when conjugate $1(5.0 \mu M)$ was treated with GSH $(5.0 \mathrm{mM})$ at $37^{\circ} \mathrm{C}$ in PBS buffer $(\mathrm{pH} 7.4)$. (d) TEM imagine of the nanoparticles after treated with $G S H$ for $2 \mathrm{~h}$, at $37^{\circ} \mathrm{C}$ in $\mathrm{PBS}$ buffer (pH 7.4). 

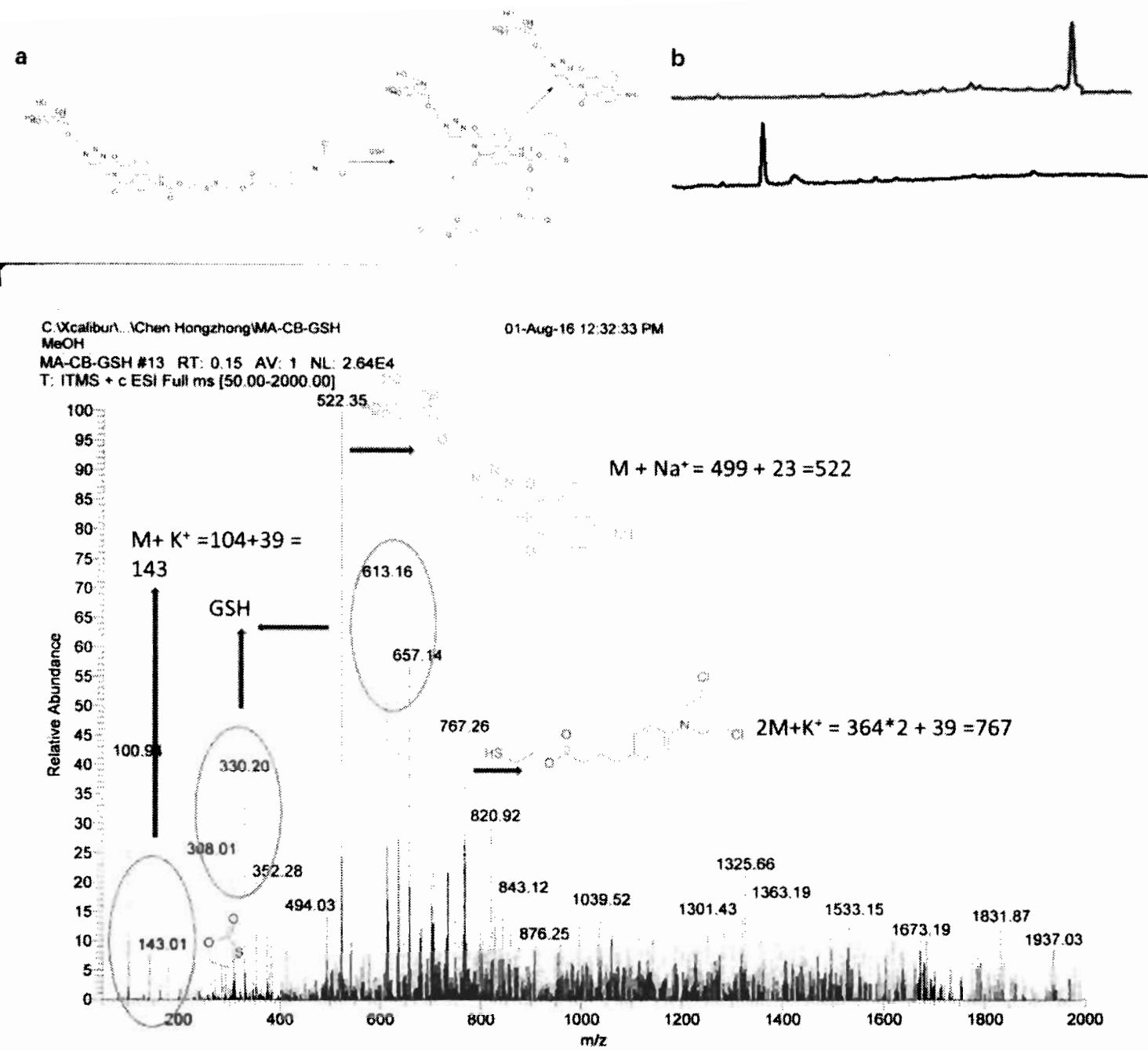

Scheme 3 (a) The GSH trigger mechanism of the conjugate 1. (b) HPLC of the conjugate 1 before and

after treated with GSH. (c) LC-MS of the conjugate 1 after treatment with GSH.

\subsubsection{Cellular uptake study}

To confirm the target specificity of the vesicles to mannose receptor overexpressed cancer cells, the vesicle was incubated with two cancer cell lines, HeLa cells and MCF-7 cells. These two cell lines were chosen due to high mannose receptor expression level of MCF-7 cells and extremely low mannose receptor expression level of HeLa cells (Figure 33). As shown in Figure 33, when treated with conjugate 1, a strong intracellular fluorescence was observed in the case of MCF-7 cells both in the 
blue and green channel by using confocal laser scanning microscope (CLSM), whereas extremely weak fluorescence was observed in the case of $\mathrm{HeLa}$ cells under the same experimental conditions. This significant difference in intracellular emissive behaviour in these two cases of cancer cell lines indicated that the selective uptake of conjugate 1 in the case of MCF-7 cells by mannose receptor mediated endocytosis. This kind of selective fluorescence responsive property gives this system the potential for imaging-guided drug delivery and potential application in bio-imaging field.

Blue

a) i)

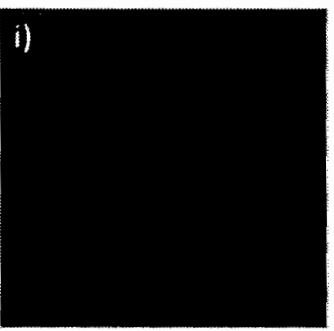

b) i)

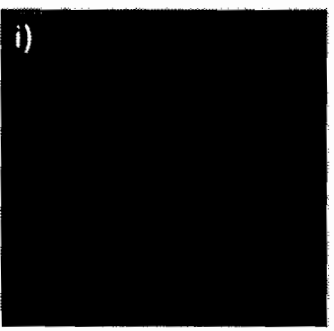

Green
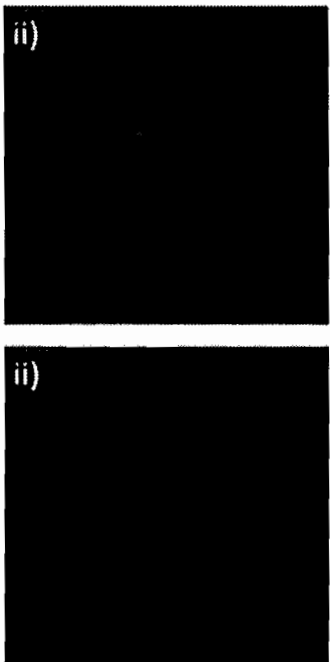

Merged
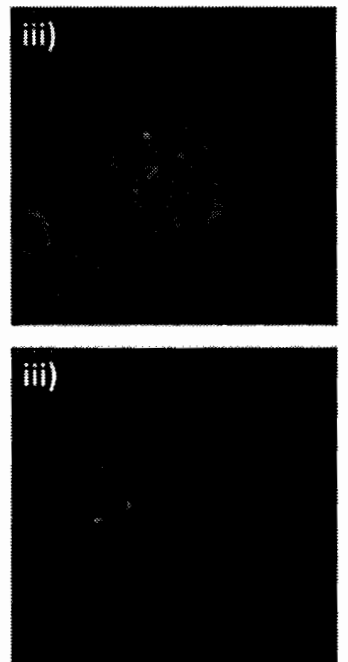

Bright

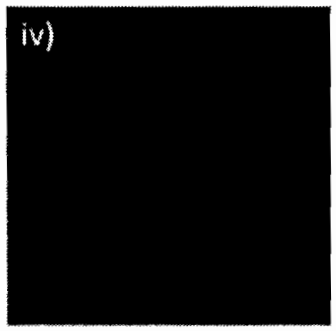

iv)

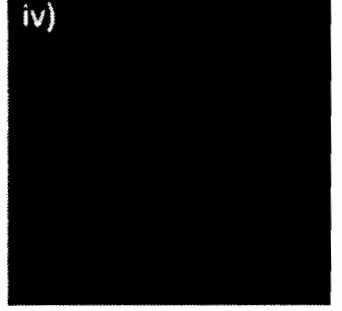

Figure 33 a) Confocal fluorescence images of MCF-7 cells: MCF-7 cells incubated with the conjugate 1

$(10 \mu \mathrm{M})$ for $24 \mathrm{~h}$ i) b/ue channel at $450 \pm 35 \mathrm{~nm}$, ii) green channel at $515 \pm 30 \mathrm{~nm}$, iii) Overlap image generated from (i) and (ii), and iii) bright-field transmission image, scale bar $=20 \mathrm{um}$.

To investigate the time-dependent intracellular fluorescence changes and ratio of blue/green fluorescence for MCF-7 cells treated with the vesicles, CLSM was also employed (Figure 34). As shown in Figure 6, after incubated with vesicle for $1 \mathrm{~h}$, the blue/green intensity ratio is $2.242,1.758$ for $12 \mathrm{~h}$, and 0.97 for $24 \mathrm{~h}$. The obvious ratio change indicated the dissociation and drug release process of the vesicles. 


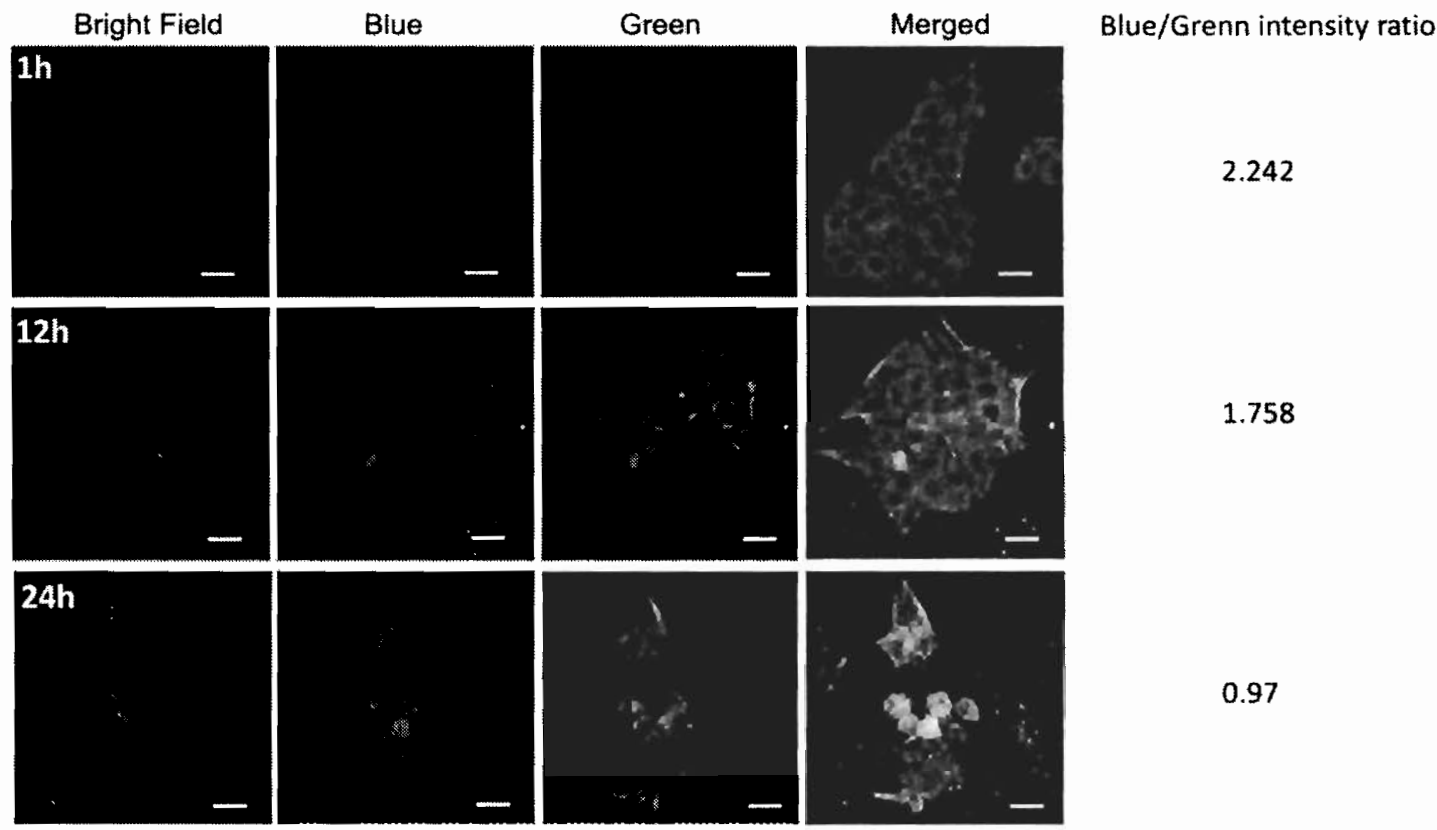

Figure 34 Time-dependent intracellular fluorescence change of the MCF-7 cells with $1 \mathrm{~h}, 12 \mathrm{~h}$, and $24 \mathrm{~h}$

and the blue/green intensity ratio in different time. From left to right, bright-field transmission image, blue channel at $450 \pm 35 \mathrm{~nm}$, green channel at $515 \pm 30 \mathrm{~nm}$. Overlap image generated from the three photos in the left side. scale bar $=20 \mu \mathrm{m}$

In order to provide further evidence of the selective endocytosis, flow cytometry was applied to study the cellular uptake in MCF-7 cells and HeLa cells. The two cell lines were incubated for $24 \mathrm{~h}$ in the presence of conjugate 1 and conjugate 2 , respectively. The obtained results are shown in Figure 35. MCF-7 cells incubated with conjugate 1 and conjugate 2 both displayed much stronger fluorescence intensity than the blank control in BV421-A and Amcyan-A channels. However, MCF-7 cells incubated with conjugate 1 exhibited stronger fluorescence intensity than cells incubated with conjugate 2 in both channels demonstrating high internalising efficiency of conjugate 1 . However, in the case of HeLa cells with low expression of mannose receptors, cells displayed almost the same fluorescence intensity in 
Amcyan-A channel after incubation with conjugates 1 and 2. In BV421-A channel, cells incubated with conjugate 2 showed only a slightly stronger fluorescence intensity than the cells incubated with conjugate 2 . These results demonstrated that conjugate 1 with grafted mannose group had better selective endocytosis than conjugate 2 with targeting properties due to the binding with mannose receptor on the cells surface. The dot plot combination of both BV421-A and AmCyan-A channels are shown in Figure 36. When MCF-7 cells were incubated with conjugate 1, there was an obvious fluorescence shift from $0 \%$ to $99.5 \%$ in Q2 whereas the shift was only to $65.1 \%$ for conjugate 2 (Figure 8). When HeLa cells were incubated with conjugate 1, the fluorescence shifted from $0.017 \%$ in Q2 for the control to $85.3 \%$ with conjugate 1 . The shift was when incubated with conjugate 2 was also similar to that of conjugate 1 $(91.9 \%)$ 

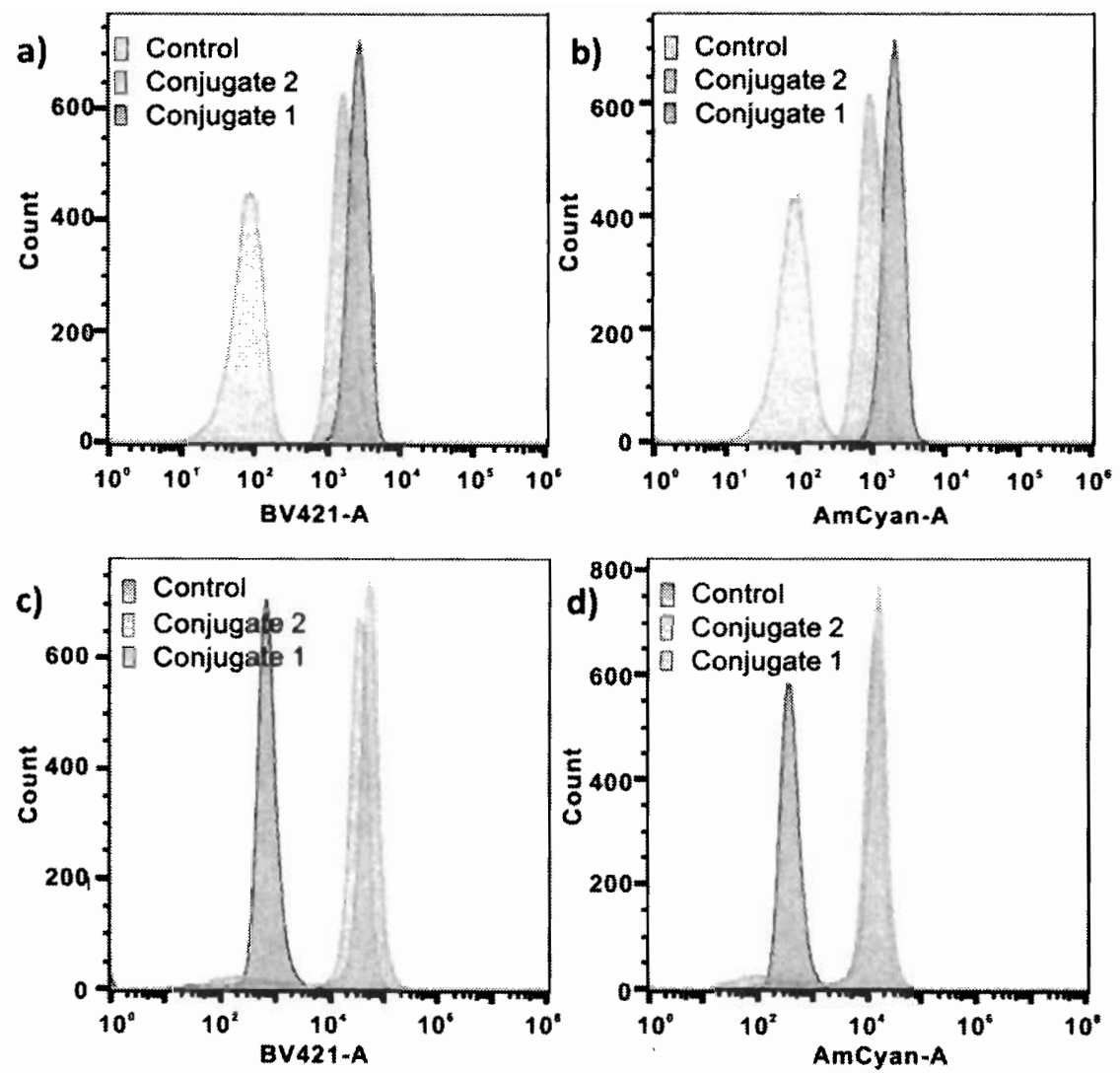

Figure 35 Flow cytometric analysis of a) BV421-A channel and b) AmCyan-A channel fluorescence intensities from MCF-7 cell line after incubation with conjugate 1 ( $25 \mu \mathrm{g} \mathrm{mL}-1)$. Flow cytometric analysis of c) BV421-A channel and d) AmCyan-A channel fluorescence inte intensities from HeLa cell line after incubation with conjugate 1 (25 $\mu \mathrm{g} \mathrm{mL}^{(-1)}$ ) 

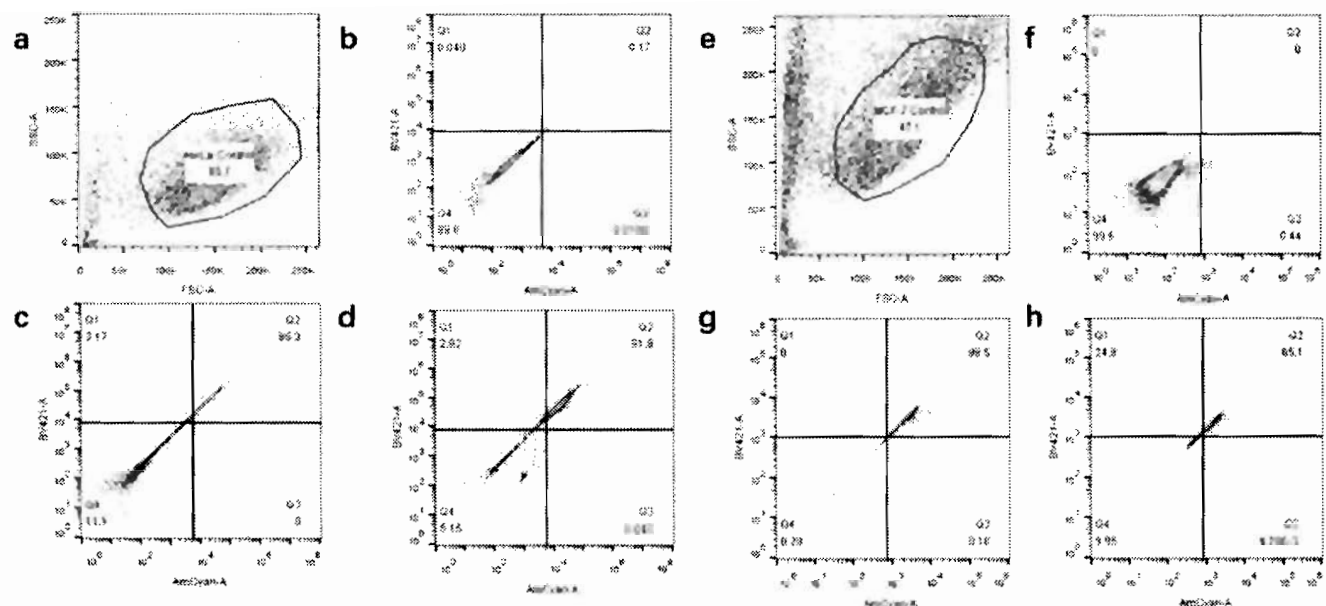

Figure 36 (a) Dot plots of live MCF-7 cell popsllation and fluorescence for (b) blank cell sample, (c) cell sample incubated with conjugate 1 for $24 h$, and (d) cell sample incubated with conjugate 2 for $24 \mathrm{~h}$ (e) Dot plots of live HeLa cell population and fluoresce fluorescence for (f) blank cell sample, (g) cell sample incubated with conjugate 1 for $24 \mathrm{~h}$, and (h) cell sample incubated with conjugate 2 for $24 \mathrm{~h}$.

\subsubsection{In vitro antitumor study}

To identify the efficiency of cell killing ability of conjugate 1 , the in vitro cytotoxicity of conjugate 1 was investigated by using MTT assay. As shown in Figure 37, a dose dependency of cell toxicity was observed $(0-400 \mu \mathrm{g} / \mathrm{mL})$, when MCF- 7 cells were treated with conjugate 1 and chlorambucil after $42 \mathrm{~h}$. The most interesting thing is that conjugate 1 exhibited higher toxicity than chlorambucil at low doses $(0-100 \mu \mathrm{g} / \mathrm{mL})$, which indicated that conjugate 1 had higher uptake efficacy than chlorambucil under physiological conditions. However, in the case of HeLa cells, chlorambucil always showed higher toxicity than conjugate 1 regardless of the dosage (Figure 37). This different cytotoxicity in the two cancer cell lines further supplemented the preferential targeting capability of the nanomaterial, which was demonstrated by the flow cytometry and CLSM results obtained, thereby confirming 
the successful construction of the targeted drug delivery systems. For mannose receptor-positive cell line MCF-7, at low concentrations $(0-100 \mu \mathrm{g} / \mathrm{mL})$, conjugate 1 was more effective in killing the cells rather than free chlorambucil, indicating more efficient uptake of conjugate 1 than free drug. However, when the same experiment was carried out on the negative control HeLa cells (Figure 37), the opposite was observed, in which the free drug displayed better cell killing effect than conjugate 1 at all concentrations. This different cytotoxicity and uptake in the two cancer cell lines supported preferential targeting capability of the vesicles, thereby confirming the successful construction of the targeted drug delivery system.
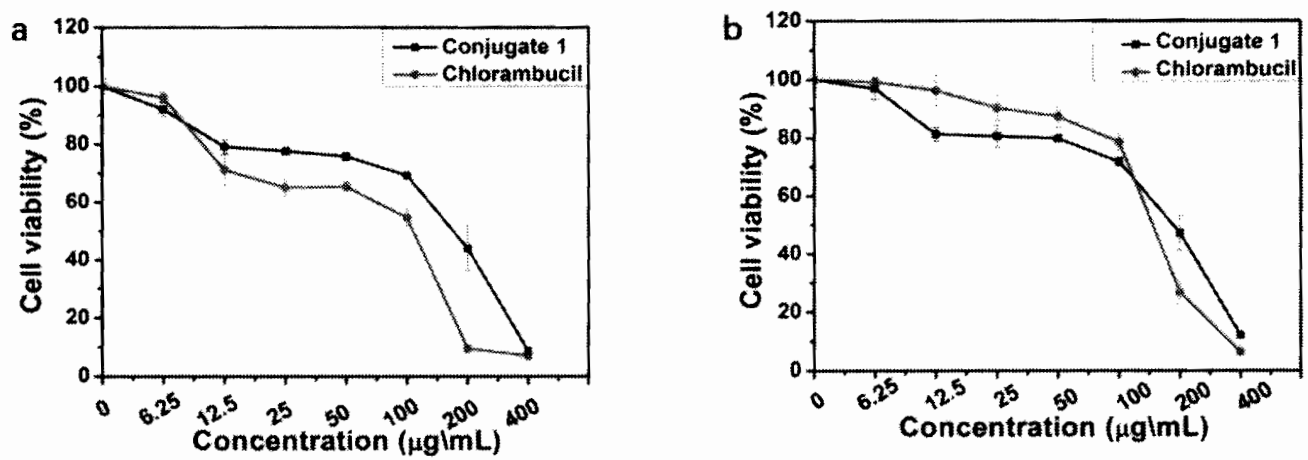

Figure 37 (a) MTT assay of HeLa cells incubated with vesicles and free chlorambucil for $72 \mathrm{~h}$. respectively. (b) Cytotoxicity of MCF-7 cells incubated with conjugated 1 and free chlorambucil at 37 "C for $72 h$

\subsection{Conclusion}

In summary, based on the principle of receptor mediated targeted delivery, we have successfully synthesized mannose-grafted chlorambucil with a naphthalimide fluorescence reporter group. We have also prepared nanosized vesicles owing to inherent amphiphilicity of the prodrug. The morphology of the nanoparticles was 
confirmed by TEM, SEM as well as DLS and the size of nanoparticles was shown to be suitable for drug delivery. After uptake by cancer cells, the nanoparticles were cleaved under the reducing environment of cancer cells accompanied with drug release and fluorescence changes. CLSM and flow cytometry results provide evidences of selective uptake of conjugate 1 in mannose receptor-overexpressed cancer cells. The obvious selective fluorescence red-shit gave the nanoparticles the possibility for intracellular imaging and imaging-guided drug delivery. The nanoparticles exhibited higher selective toxicity in mannose receptor-overexpressed cancer cells and better performance than original drug, chlorambucil. This nano-platform showed excellent targeted imaging-guided drug delivery ability and has great potential application in cancer therapy. In the future in vivo studies, due to macrophages also overexpression mannose receptor, we would like to introduce PEG modified prodrug to reduce potential macrophage uptake problem by using co-assembly strategy. The related experiments have been under the process. 


\section{Chapter 3: Dendritic Supramolecular Theranostic Prodrug Vesicle Delivery of siRNA for Responsive Imaging Guided Synergetic Cancer Therapy}

\subsection{Introduction}

Chemotherapy as a dominant therapeutic approach was widely used in clinical cancer treatment. Compared with the surgery and radiotherapy, chemotherapy exhibits an obvious advantage which is systemic treatment, due to chemotherapeutic agents endowed to be distributed at the most of organs and tissues along with the blood circulation process. On the other hand, wide distribution of the anticancer agents leads to some serious drawbacks including lack of specificity and other side effects. To overcome the drawbacks of the chemotherapy as mentioned above, construction of the prodrugs has become an increasingly popular strategy and attracted lots of attention. Prodrugs are post-modification drugs which show minimum toxicity and can be converted to the effective form after uptake by tumor or activated by external stimuli.

Prodrugs not only reduce the side effects, but also improve the water solubility and extend circulation time. The most important thing is that prodrug can be selectively activated because of specific trigger groups introduced into the structure, which significantly enhance the selectivity of the chemotherapy. Several functional trigger groups have been developed including external stimuli, for example light, and tumor microenvironments responsive such as low $\mathrm{pH}$ value, high reactive oxygen species (ROS) concentration, high glutathione (GSH) concentration. GSH is an important 
antioxidant in human body, which can prevent the damage caused by ROS. Interestingly, the concentration of the GSH was high in many types of cancer cells as compared to normal cells, which allows the design and fabrication of GSH-responsive prodrug by introducing disulfide bond as functional linker.

To achieve more accurate and effective therapy, theranostic prodrugs are developed by introducing fluorophores into the prodrugs. Theranostic prodrugs undergo fluorescence changes including fluorescence turn on or bathochromic shift after triggered by the intracellular or external stimuli, which can be utilized to precisely monitor the release process in real time. This fluorescence changes are also employed to image cancer cells and the distribution of the prodrugs. Similar with the common prodrugs, the theranostic prodrugs are also constructed by using similar stimuli responsive functional linkers such as GSH, ROS, and $\mathrm{pH}$.

Particles with the size distribution below the $200 \mathrm{~nm}$ prefer to accumulate at the tumor tissues rather than normal tissues, due to mutation architecture of the vessels in tumor tissues in EPR effect. In order to enhance the tumor tissues uptake efficacy, prodrugs are encapsulated inside the nanosized carriers or directly self-assembled into nanoparticles. Nanoparticles loaded prodrugs are faced with some common disadvantages of the drug delivery systems (DDS) including poor drug loading content, premature drug leakage, and insufficient drug release. Alternate approach of forming self-assemblies nanoparticles by amphiphilic prodrugs has drawn lots of attention. However it is faced with many challenges such as water solubility as well as the stability of the prodrug, and complicated synthesis and time-consuming 
purification steps. The concept of supraamphiphiles that are constructed by noncovalent interactions including host-guest interactions, hydrogen bonding, and $\pi-\pi$ interactions, has been used in constructing drug delivery vectors. Among the few noncovalent interactions mentioned above, the host-guest interactions based on macrocycles such as cyclodextrin, cucurbituril, sulfonatocalixarene and pillararene, are the most frequently employed techniques, because of the high biocompatibility of the macrocycles and ease in fabrication of stable water-soluble supramolecular architectures. In addition, generation of the prodrug assemblies efficiently avoid the usage of the complicated organic synthesis.

In recent years, small interfering RNA (siRNA) based on gene therapy has drawn much attention, because siRNA can efficiently inhibit diseases related genes expression and silence specific proteins, achieving a gene therapeutic effect. However, the main challenge of siRNA therapy is to find safe and efficient delivery vectors. Although viral vectors exhibit highly transfection efficacy, their potential immune responses, inflammatory and gene control effect leads to safety consideration. Non-viral alternatives developed in past decades are cationic lipids and polymers. Both of them have their own drawbacks in which the lipids are limited to be used in in vivo applications due to highly toxicity and polymers are frustrated because of undefined structural composition. Development of the non-viral vectors without the limitations of the cationic lipids and polymers has been a hot topic for research and attracted a lot of attention in gene therapy. Furthermore, it has been reported that siRNA targeting Plk1 could improve the therapeutic efficacy of the CPT 
(camptothecin) in treatment of the cancer. In account of the working mechanism, siRNA should be released faster than the chemo-drugs, so construction of the prodrugs is a wise strategy due to its controlled release manner $20,66,200-205$.

Herein, benefiting from the concept of supraamphiphiles, we developed a dendritic supramolecular theranostic prodrug vesicle based on host-guest interactions, and employed this vesicle as a vector to deliver the siRNA in Scheme 4. The fluorescence of the prodrug underwent red-shift after triggered by GSH, which was used to monitor the drug release in real time and also utilized in intracellular imaging. The structures of the host and guest molecules were shown below. Dendrimer (H1) was grafted on $\beta$-cyclodextrin via click reaction to give the host molecule ( $\mathrm{H} 2$ ). Adamantane (Ada) attached naphthalimide modified CPT was treated as guest molecule. The Ada segment was included by the cyclodextrin, resulting in the formation of the supramphiphiles, following with spontaneous self-assembly to obtain supramolecular vesicles with high prodrug loading content of $25.7 \%$. The siPlK1 was loaded by mixing the siRNA with the vesicle solution, and the obtained nanoparticles were incubated with the cancer cells. After internalization by the cancer cells, the siRNA escaped from the lysosome and the prodrug was cleaved by GSH, followed by intramolecular cyclization to afford the free CPT, while the fluorophore underwent the similar intramolecular cyclization to release compound 3 accompanied by significant fluorescence red-shift. This fluorescence change was employed to monitor the drug release in real time and imaging for the cancer cells. Our study provides a new strategy to co-deliver prodrug with siRNA. 
a)
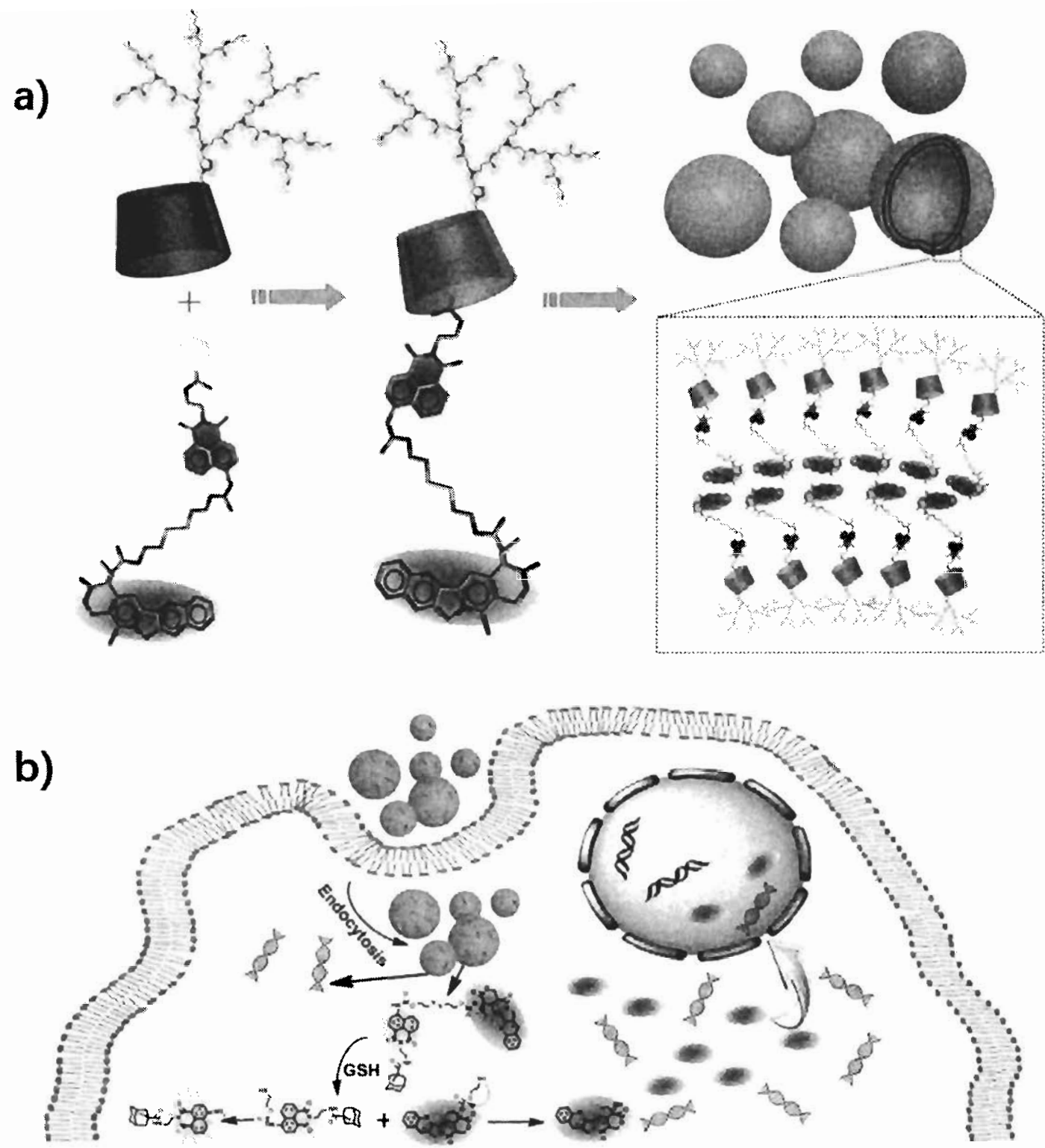

Scheme 4 (a) Schematic illustration of the self-assembly process. (b) Schematic illustration of the

therapeutic agent release and fluorescence turn on mechanism.

\subsection{Materials and Characterizations}

THF and toluene were refluxed with sodium overnight before used. DCM and was refluxed with $\mathrm{CaH}_{2}$ overnight before used. DMF was refluxed with $\mathrm{CaH}_{2}$ overnight and distilled under the reduced pressure before used. Ethanol was refluxed with 
magnesium and iodine overnight and distilled before used. SiPlK1 with the sequence of (sense strand, 5-UGAAGAAGAU-CACCCUCCUUAdTdT-3; antisense strand, 5-UAAGGAGGGUGAUCUUCUUCAdTdT-3) and scrambled siRNA (siNonsense) with the sequence of (sense strand, 5-UUCUCCGAACGUGUCACGUdTdT-3; antisense strand, 5-ACGUGACACGUUCGGAGAAdTdT-3) were supplied by Su Zhou Ribo Life Science Co. Ltd. (Suzhou, China). Cy3 labeled siRNA with the sequence of (sense strand, 5'-UUCUCCGAACGUGUCACGUdTdT-'3; antisense strand, 5'-ACGUGACACGUUCGGAGAAdTdT-3', labeled with cy3 at 5 position) was supplied from shanghai Genepharma (Shanghai China). Adamantanecarboxylic acid chloride, Palladium on carbon, hydrazine hydrate (70\%-80 \%), N,N-Diisopropylethylamine (DIPEA), triphosgene, 2, 2 '-dithiodiethanol , 4-dimethylaminopyridine (DMAP), sodium azide, propargylamine, 4-nitro-1,8-naphthalic anhydride, Di-tert-butyl pyrocarbonate, methyl acrylate, ethylenediamine, and L-ascorbic acid sodium salt were obtained from Sigma Aldrich and were used without further purification. The cyclodextrin was a gift given by my friend. Dendrimer $\mathrm{H} 1$ and 4-Nitro-N-(2-aminoethyl)-1,8-naphthalimide were synthesized according the literature reported procedures ${ }^{201,206-208}$

${ }^{1} \mathrm{H}$ and ${ }^{13} \mathrm{C}$ nuclear magnetic resonance (NMR) spectrum was measured on a Bruker BBFO-400 spectrometer using deuterated chloroform $\left(\mathrm{CDCl}_{3}\right)$ and deuterated dimethyl sulphoxide (DMSO- $\mathrm{d}_{6}$ ) as the solvents. The electronic spray ionization (ESI) mass spectra were recorded on a ThermoFinnigan LCQ quadrupole ion trap mass spectrometer. High-resolution mass spectrometry (HR-MS) was performed on a 
Waters Q-tof Premier MS spectrometer. Transmission electron microscopy (TEM) images were collected on JEM-1400 (JEOL) at $100 \mathrm{kV}$. The fluorescence emission spectra were recorded on a Shimadzu RF-5301pc fluorescence spectrophotometer. UV-vis spectra were recorded from Shimadzu UV-3600 spectrophotometer. Zeta-potential values were determined by Malvern Instruments Zetasizer Nano-S at $25^{\circ} \mathrm{C}$. DLS size distributions were measured on a Nanobrook 90Plus particle size analyzer. MTT was recorded on a Tecan Infinite M200. The drug release behavior was determined by HPLC. CLSM was recorded on a Carl Zesis LSM 800. Gel retardation assay was recorded on Horizontal Electrophoresis systems, Bio-Rad, Singapore.

\subsubsection{Synthesis of the compounds}

\subsubsection{Preparation of host molecule dendrimer grafted cyclodextrin}

$\mathrm{H} 1(1.6 \mathrm{~g}, 1 \mathrm{mmol})$ and $\beta$-CD-N3 $(1.6 \mathrm{~g}, 1.38 \mathrm{mmol})$ were dissolved in the $\mathrm{H}_{2} \mathrm{O}(40$ $\mathrm{mL})$. CuSO4.5H2O (750 mg, $3 \mathrm{mmol}$ ) was added into the solution. The solution was then stirred at room temperature, L-ascorbic acid sodium salt $(2 \mathrm{~g}, 10 \mathrm{mmol})$ was added into the solution in portions over a period of $1 \mathrm{~h}$ under protection of inert $\mathrm{N}_{2}$ gas. The solution was stirred under $\mathrm{N}_{2}$ atmosphere at $50{ }^{\circ} \mathrm{C}$ for $48 \mathrm{~h}$. After cooling to room temperature, the mixture was filtered to remove copper (I) compound, and the filtrate was dialysis against water for 5 days using dialysis of cut-off MW 2000. The water was removed by lyophilization to give the $\mathrm{H} 2$ as beige solid $(920 \mathrm{mg}, 32.8 \%) .{ }^{1} \mathrm{H}$ NMR $\left(400 \mathrm{MHz}, \mathrm{D}_{2} \mathrm{O}, 298 \mathrm{~K}\right): \delta 8.02(\mathrm{~s}, 1 \mathrm{H}), 4.9 \sim 5.3(\mathrm{~m}, 14 \mathrm{H}), 2.32 \sim 4.05(\mathrm{~m}$,

155H). ${ }^{13} \mathrm{C} \mathrm{NMR}\left(100 \mathrm{MHz}, \mathrm{CDCl}_{3}, 298 \mathrm{~K}\right): \delta 178.6,164.0,163.0,149.7,132.6$, 
$130.0,129.5,129.2,126.7,123.9,123.7,122.9,40.5,40.1,39.0,36.4,28.0$. HRMS

(TOF) $\mathrm{m} / \mathrm{z}[\mathrm{M}+\mathrm{H}]^{+}$, calcd for $\mathrm{C}_{115} \mathrm{H}_{214} \mathrm{~N}_{32} \mathrm{O}_{48}, 2812.5367$; found, 2812.5264 .

\subsubsection{Synthesis of the compound 4}

To a solution of compound $5(1.85 \mathrm{~g}, 6.5 \mathrm{mmol})$ in dry DCM $(20 \mathrm{~mL})$, TEA $(1.3 \mathrm{~g}$, $13 \mathrm{mmol}$ ) was added, and the mixture was allowed stirred under the ice bath. Adamantanecarboxylic acid chloride $(2 \mathrm{~g}, 10 \mathrm{mmol})$ was dissolved in dry DCM (10 $\mathrm{mL}$ ) was dropwise added into the solution over $30 \mathrm{~min}$. Then the solution was allowed to stir under the room temperature overnight. The solution was washed with water for 3 times and then organic layer was collected and dried against anhydrous sodium sulfate. After removal of the solvents, the crude product was purified over silica gel chromatography using hexane/ethyl acetate $(\mathrm{v} / \mathrm{v}, 1: 1)$ as the eluent. The solvents were removed to afford the product 4 as beige solid $(2.4 \mathrm{~g}, 82.8 \%)$. ${ }^{1} \mathrm{H}$ NMR (400 MHz, $\left.\mathrm{CDCl}_{3}, 298 \mathrm{~K}\right): \delta 8.85(\mathrm{~d}, 1 \mathrm{H}), 8.73(\mathrm{dd}, 2 \mathrm{H}), 8.41(\mathrm{~d}, 1 \mathrm{H}), 8.00(\mathrm{t}, 1 \mathrm{H}), 6.17(\mathrm{~s}, 1 \mathrm{H})$, $4.42(\mathrm{~m}, 2 \mathrm{H}), 3.69$ (s. $2 \mathrm{H}), 1.96(\mathrm{~s}, 3 \mathrm{H}), 1.78 \sim 1.68(\mathrm{~m}, 12 \mathrm{H}) .{ }^{13} \mathrm{C}$ NMR $(100 \mathrm{MHz}$, $\mathrm{CDCl}_{3}, 298 \mathrm{~K}$ ): $\delta$. HRMS (TOF) $\mathrm{m} / \mathrm{z}[\mathrm{M}+\mathrm{H}]^{+}$, calcd for $\mathrm{C}_{25} \mathrm{H}_{25} \mathrm{~N}_{3} \mathrm{O}_{5}, 448.1872$; found, 448.1877.

\subsubsection{Synthesis of the compound 3}

To a stirred cloudy solution of compound $4(1 \mathrm{~g}, 2.23 \mathrm{mmol})$ in ethanol, catalytic amount palladium on carbon was added. The hydrazine hydrate $(70-80 \%, 2 \mathrm{~mL})$ was then added, and the solution was stirred under the reflux overnight. After cooling to room temperature, the solution was filter to remove $\mathrm{Pd} @ \mathrm{C}$, and the filtrate was collected and evaporated under the reduced pressure to afford the crude product. The 
crude product was purified by silica gel chromatography using hexane/ethyl acetate $(\mathrm{v} / \mathrm{v}, 1: 1)$ as the eluent. The solvents were removed to afford the product 3 as light yellow solid (700 mg, 75\%). ${ }^{1} \mathrm{H}$ NMR (400 MHz, $\left.\mathrm{CDCl}_{3}, 298 \mathrm{~K}\right): \delta 8.60(\mathrm{~d}, 1 \mathrm{H}), 8.41$ $(\mathrm{d}, 1 \mathrm{H}), 8.12(\mathrm{~d}, 1 \mathrm{H}), 7.64(\mathrm{t}, 1 \mathrm{H}), 6.87(\mathrm{~d}, 1 \mathrm{H}), 6.53(\mathrm{~s}, 1 \mathrm{H}), 5.05(\mathrm{~s}, 2 \mathrm{H}), 4.41(\mathrm{~m}$, 2H), $3.63($ dd. $2 \mathrm{H}), 1.98(\mathrm{~s}, 3 \mathrm{H}), 1.77(\mathrm{~d}, 6 \mathrm{H}), 1.66(\mathrm{t}, 6 \mathrm{H}) .{ }^{13} \mathrm{C}$ NMR $(100 \mathrm{MHz}$ $\left.\mathrm{CDCl}_{3}, 298 \mathrm{~K}\right): \delta 178.5,165.2,164.6,134.1,131.7,130.0,127.3,124.9,122.8,120.0$, $111.5,109.5,40.5,39.9,39.1,36.6,28.2$. HRMS (TOF) $\mathrm{m} / \mathrm{z}[\mathrm{M}+\mathrm{H}]^{+}$, calcd for $\mathrm{C}_{25} \mathrm{H}_{27} \mathrm{~N}_{3} \mathrm{O}_{3}, 418.2131$; found, 418.2148 .

\subsubsection{Synthesis of the compound 2}

To a mixture of compound 3 (208 $\mathrm{mg}, 0.5 \mathrm{mmol})$, and DIPEA (194 $\mathrm{mg}, 1.5 \mathrm{mmol})$, in $10 \mathrm{~mL}$ anhydrous toluene was dropwisely added a solution of triphosgene (445 $\mathrm{mg}$, $1.5 \mathrm{mmol}$ ), in $8 \mathrm{~mL}$ toluene. The mixture was refluxed for $3 \mathrm{~h}$ and then allowed to cooling to room temperature. The solvent was removed under the reduced pressure. Then a solution of 2, 2 '-dithiodiethanol $(1.23 \mathrm{~g}, 4 \mathrm{mmol})$ in $30 \mathrm{~mL}$ anhydrous THF was added to the flask. The reaction mixture was stirred overnight under the room temperature. The solvents were evaporated, at which point DCM $(100 \mathrm{~mL})$ and water $(100 \mathrm{~mL})$ were added, and the organic layer was collected. The DCM layer was dried using anhydrous $\mathrm{NaSO}_{4}$. After removal of the solvents, the crude product was purified over silica gel using hexane/ethyl acetate $(\mathrm{v} / \mathrm{v}, 3: 1)$ as the eluent to remove the impurity and then changed to hexane/ethyl acetate ( $\mathrm{v} / \mathrm{v}, 1: 1)$ as the eluent to yield 3 as a light yellow solid (300 mg, 65.5\%). ' $\mathrm{H} \mathrm{NMR}$ (400 MHz, $\mathrm{CDCl}_{3}, 298 \mathrm{~K}$ ): $\delta 8.59$ (m, 2H), $8.36(\mathrm{~d}, 1 \mathrm{H}), 8.26(\mathrm{~d}, 1 \mathrm{H}), 7.88(\mathrm{~s}, 1 \mathrm{H}), 7.73(\mathrm{t}, 1 \mathrm{H}), 6.37(\mathrm{~s}, 1 \mathrm{H}), 4.57(\mathrm{t}, 2 \mathrm{H})$, 
$4.41(\mathrm{~m}, 2 \mathrm{H}), 3.95(\mathrm{~d} .2 \mathrm{H}), 3.65(\mathrm{dd}, 2 \mathrm{H}), 3.08(\mathrm{t}, 2 \mathrm{H}), 2.96(\mathrm{t}, 2 \mathrm{H}), 2.35(\mathrm{~s}, 1 \mathrm{H}), 1.97$

$(\mathrm{s}, 3 \mathrm{H}), 1.73(\mathrm{t}, 6 \mathrm{H}), 1.66(\mathrm{t}, 6 \mathrm{H}) .{ }^{13} \mathrm{C} \mathrm{NMR}\left(100 \mathrm{MHz}, \mathrm{CDCl}_{3}, 298 \mathrm{~K}\right): \delta 178.5,164.6$, $164.3,153.1,139.4,132.7,131.5,129.0,16.6,123.4,117.3,117.0,63.9,60.0,41.6$, $40.5,39.5,39.4,39.1,37.6,36.5,28.1$. HRMS (TOF) $\mathrm{m} / \mathrm{z}[\mathrm{M}+\mathrm{H}]^{+}$, calcd for $\mathrm{C}_{30} \mathrm{H}_{35} \mathrm{~N}_{3} \mathrm{O}_{6} \mathrm{~S}_{2}$, 598.2046; found, 598.2047.

\subsubsection{Synthesis of the guest molecule G1 adamantane modified CPT}

Camptothecin (100 mg, $0.287 \mathrm{mmol})$ and DMAP (110 $\mathrm{mg}, 0.9 \mathrm{mmol})$ were dispersed in dry DCM $(15 \mathrm{~mL})$. Then triphosgene $(30 \mathrm{mg}, 0.1 \mathrm{mmol})$ was added into the mixture, and the mixture was allowed to stir for $30 \mathrm{~min}$. During this period, the mixture turned from turbid to transparent. Compound $2(158 \mathrm{mg}, 0.264 \mathrm{mmol})$ dissolved in dry DCM ( $5 \mathrm{~mL})$ was dropwise added into the solution. The solution was allowed stirred under the room temperature for overnight. The solution was washed with water for 3 times and dried over anhydrous sodium sulfate. The solvents were removed under the reduced pressure to give crude product. The crude produce was purified by silica gel chromatography using ethyl acetate as eluent. The solvents were removed under the vacuum to afford the pure product as light yellow powder $(245 \mathrm{mg}$, 95.35\%). ${ }^{1} \mathrm{H}$ NMR $\left(400 \mathrm{MHz}, \mathrm{CDCl}_{3}, 298 \mathrm{~K}\right): \delta 8.55(\mathrm{t}, 2 \mathrm{H}), 8.41(\mathrm{~s}, 1 \mathrm{H}), 8.29(\mathrm{~d}$, $1 \mathrm{H}), 8.20(\mathrm{dd}, 2 \mathrm{H}), 8.04(\mathrm{~s}, 1 \mathrm{H}), 7.96(\mathrm{~d}, 1 \mathrm{H}), 7.85(\mathrm{t}, 1 \mathrm{H}), 7.68(\mathrm{dd}, 2 \mathrm{H}), 7.30,(\mathrm{~s}$, 1H), $6.51(\mathrm{~s}, 1 \mathrm{H}), 5.2 \sim 5.05(\mathrm{dd}, 4 \mathrm{H}), 4.6 \sim 4.3(\mathrm{~m}, 6 \mathrm{H}), 3.69(\mathrm{~d} .2 \mathrm{H}), 3.08(\mathrm{~m}, 4 \mathrm{H})$, $2.96(\mathrm{t}, 2 \mathrm{H}), 2.13(\mathrm{ddd}, 2 \mathrm{H}), 1.98(\mathrm{~s}, 3 \mathrm{H}), 1.79(\mathrm{~s}, 6 \mathrm{H}), 1.66(\mathrm{~m}, 6 \mathrm{H}), 0.98(\mathrm{t}, 3 \mathrm{H}) .{ }^{13} \mathrm{C}$ NMR (100 MHz, $\left.\mathrm{CDCl}_{3}, 298 \mathrm{~K}\right): \delta=178.45,167.22,164.78,164.35,156.91,153.88$, $152.89,152.02,148.88,146.47,145.70,139.56,132.54,131.33,130.89,129.55$, 
$128.96,128.33,127.06,126.42,123.00,119.70,117.49,116.71,95.88,77.93,66.61$, $62.76,49.96,40.49,39.42,39.04,37.67,36.56,32.29,31.71,28.13,7.65$. HRMS (TOF) $\mathrm{m} / \mathrm{z}[\mathrm{M}+\mathrm{H}]^{+}$, calcd for $\mathrm{C}_{51} \mathrm{H}_{49} \mathrm{~N}_{5} \mathrm{O}_{11} \mathrm{~S}_{2}, 972.2948$; found, 972.2905.

\subsubsection{Preparation of vesicles and siRNA loading vesicles}

Guest prodrug was dissolved in DMSO to prepare a stock solution with the concentration of $4 \mathrm{mM}$. The dendritic cyclodextrin was dissolved in DI water to obtain a stock solution with the concentration of $2 \mathrm{mM}$. The stock solution of the host molecule was diluted 20 folds to $100 \mu \mathrm{M}$. Upon sonication, $25 \mu \mathrm{L}$ guest stock solution was slowly added into the above solution $(975 \mu \mathrm{L})$ over 5 min to obtain a cloudy solution. The solution was allowed to stand for overnight at room temperature for sufficient assembly, followed by dialysis against water for $2 \mathrm{~h}$ to remove trace DMSO. The obtained solution was stored at $4{ }^{\circ} \mathrm{C}$. To prepare the siRNA loading vesicles, the siPIK1 was added into the fresh prepared vesicles solution, followed by shaking to mix well, and allowed to stay at $37{ }^{\circ} \mathrm{C}$ over 30 min for sufficient binding.

\subsubsection{Gel retardation assay and siRNA/vesicles complex protection assay}

For the gel retardation assay, siRNA1 and vesicles with proper concentration was prepared. For each tube, $200 \mathrm{ng}$ of siRNA was added with the respective amount of polymer base on $\mathrm{N} / \mathrm{P}$ ratio of $1 / 2.5,1 / 1,5 / 1,10 / 1,15 / 1,20 / 1$ and $10 / 1$ respectively. The final volume of each tube was made up to $10 \mu \mathrm{L}$ with Nuclease Free $\mathrm{H}_{2} \mathrm{O}$. The samples were incubated at $37^{\circ} \mathrm{C}$ for $30 \mathrm{~min}$ before running on $1.2 \%$ agarose gel. Bio-Rad Gel-Doc was used for the observation of gel image. 
For the siRNA/vesicles complex protection assay, siRNA and vesicles was incubated at $37^{\circ} \mathrm{C}$ for $30 \mathrm{~min}$. RNaseA was added with final concentration of 0.01 $\mu \mathrm{g} / \mu \mathrm{L}$, withdraw $4 \mu \mathrm{L}$ of the mixture (with $200 \mathrm{ng}$ of siRNA) out into $1.5 \mu \mathrm{L}$ of $1 \%$ SDS and place on ice at time $0,5,10,15,20,30,45,60,75,90,105$ and 120 min. For naked siRNA1, the same procedure was carried out only without incubation in the mixture with vesicles. Samples collected at different time points were analyzed using electrophoresis with $2 \%$ agarose gel.

\subsubsection{Drug release behavior}

The drug release behavior was investigated by using HPLC. The vesicle solution was prepared via using PBS buffer with $\mathrm{pH} 7.4$ with the concentration of $100 \mu \mathrm{M}$, following in adding the GSH to the concentration of $5 \mathrm{mM}$. The mixture was incubated under the $37{ }^{\circ} \mathrm{C}$ over $200 \mathrm{~min}$. The $100 \mu \mathrm{L}$ solution was drawn out and diluted with methanol to the concentration to $20 \mu \mathrm{M}$. Acetonitrile and water were employed as eluents of the HPLC, and the ratio of the two kinds of the solvents was changed along the time. The ratio of the acetonitrile to water was 95 to 5 at $0 \sim 25 \mathrm{~min}$, and 5 to 95 at $25 \sim 32 \mathrm{~min}$, finally 95 to 5 till $40 \mathrm{~min}$. The monitored absorption wavelength was $270 \mathrm{~nm}$.

\subsubsection{Cellular uptake study}

HeLa cells were cultured with Dulbecco's modified eagle medium (DMEM) with $10 \%$ fetal bovine serum (FBS), $1 \%$ penicillin and streptomycin (PS) under $5 \% \mathrm{CO}_{2}$ atmosphere at $37^{\circ} \mathrm{C}$. 
In confocal studies, HeLa cells were seeded in a 6 -well tissue culture plate $(2 \mathrm{~mL}$ medium) with a density of $2.0 \times 10^{5}$ cells per well on the slides. After culturing $24 \mathrm{~h}$, the vesicle was added into the culture medium with the final concentration of $2 \mu \mathrm{M}$. After incubated with vesicles at different time, the culture medium was removed, following washing with the PBS for 3 times and then fixed with $4 \%$ formaldehyde at room temperature for $15 \mathrm{~min}$.

\subsubsection{In vitro cytotoxicity study}

MTT assay was used to investigate the cytotoxicity of prodrug, supramolecular amphiphiles prodrug and free drug CPT. HeLa cells were seeded in 96-well plates ( $200 \mu \mathrm{L}$ medium) and incubated for $24 \mathrm{~h}$. After the cell density reached $60 \%-70 \%$, the cells were incubated with the prodrug, supramolecular amphiphiles prodrug and free drug CPT and incubated $72 \mathrm{~h}$, respectively. After the medium removed, the fresh medium with $10 \%$ MTT was added, and incubated with for another $4 \mathrm{~h}$. The medium was removed carefully, following with adding $100 \mu \mathrm{L}$ DMSO. Finally, optical densities of the samples were measured using a microplate reader at the wavelength of both $570 \mathrm{~nm}$ and $490 \mathrm{~nm}$.

\subsection{Results and discussion}

\subsubsection{Design and synthesis of the supramolecular amphiphiles prodrug}

The synthetic procedure of the dendritic cyclodextrin and adamantane prodrug was shown in scheme 5. Dendritic $\mathrm{H} 1$ was linked on the $\beta$-cyclodextrin via $\mathrm{Cu}$ (I) mediated azide-alkyne cycloadditions, giving hydrophilic dendritic cyclodextrins as host molecules, Scheme 1 (a). Compound 6 and 4 was synthesized by adapting 
procedures reported previously. For the synthesis of the compound 4, compound 5 was reacted with adamantanecarboxylic acid chloride in the presence of TEA in dichloromethane. Compound 4 was then reduced by hydrazine hydrate in the presence of $\mathrm{Pd} @ \mathrm{C}$ in ethanol, to give compound 3. Compound 3 was reacted with triphosgene, following with reacting with $2,2^{\prime}$-dithiolethanol, to give compound 2 . At last, compound 2 was reacted with triphosgene, following with reacting with camptothecin in presence of DMAP, to afford compound G1 as guest molecules. The overall chemical structures of $\mathrm{H} 1,4,3,2$, and G1 were confirmed by ${ }^{1} \mathrm{H}$ NMR, ${ }^{13} \mathrm{C}$ NMR, and HRMS.

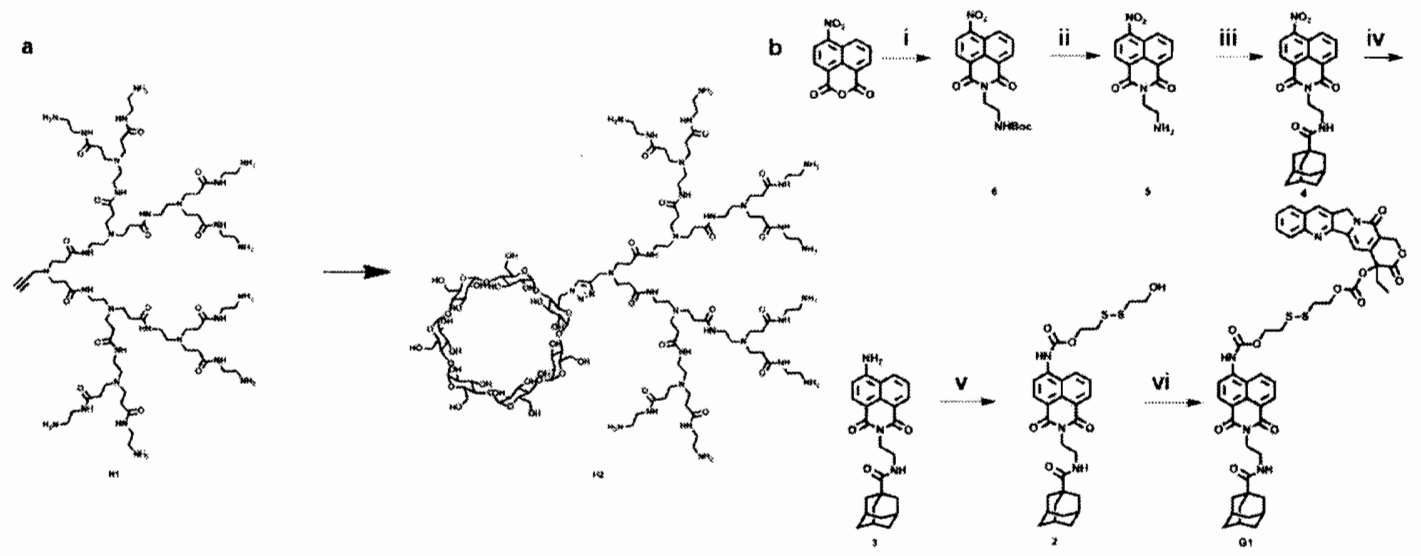

Scheme 5 (a) CuSO4.5H2O, L-Ascorbic acid sodium salt, H2O, N2, $50^{\circ} \mathrm{C}, 48$ h. (b) i: ethanol, N-boc-ethylenediamine, reflux, 6 h. ii: TFA, DCM, rt, 3 h. ii: Adamantanecarboxylic acid chloride, TEA $D C M .0^{\circ} \mathrm{C}$ to it, overnight. $\mathrm{N}:$ Pd@C, hydrazine hydrate, ethand, reflux, overnight. $v$ : DIPEA, triphosgene, 2, 2 -dithiodiethanol, THF/DCM. vi: camptothecin, DMAP, triphosgene, rt, overnight.

\subsubsection{Self-assembly behaviors of the supramolecular amphiphiles prodrug}

Due to supramolecular amphiphilic property, the host-guest inclusion complex can form higher order aggregates in water. To prepare amphiphilic supramolecular 
assembly, the Ada-prodrug dissolved in DMSO was slowly injected into the dendritic cyclodextrin solution under the sonication with the ratio of $1: 1$, followed by dialysis against water. Dynamic light scattering (DLS) was employed to determine the size of the aggregate of the $\mathrm{H} 1$ and G1s in aqueous solution. As shown in Figure 38 (c), the vesicles exhibit an average hydrodynamic diameter about $120 \mathrm{~nm}$ with a narrow distribution. Transmission electron microscopy (TEM) images exhibited vesicle like morphology with the size about $110 \mathrm{~nm}$, which was in accordance with the results of the DLS, Figure 38 (a). The size of the vesicles was around $100 \mathrm{~nm}$, which is suitable for passive uptake by tumor tissue. The wall thickness of the vesicles was about $8 \mathrm{~nm}$, which was consistent with the simulation results, Figure 39. The critical aggregation concentration (CAC) of host-guest inclusion complex was also determined by monitoring the hydrodynamic diameter $\left(\mathrm{D}_{\mathrm{H}}\right)$ changes upon increasing the concentration of the prodrug supramolecular amphiphilic vesicles range from $0.1 \mu \mathrm{M}$ to $40 \mu \mathrm{M}$. Negligible hydrodynamic diameter changes were observed for the amphiphilic prodrug supramolecular amphiphilic vesicles solution upon increasing the concentration, until the concentration of the prodrug supramolecular amphiphilic vesicles reached to $1 \mu \mathrm{M}$. An obvious size change was detected at the concentration of $1 \mu \mathrm{M}$, indicating that higher order aggregates were formed by the prodrug supramolecular amphiphilic vesicles in the aqueous solution. The solution of the prodrug supramolecular amphiphilic vesicles with the concentration above the CAC displayed obvious Tyndall effect, suggesting that plenty of nanosize aggregates was 
formed by the host-guest complex Figure 38 (d). The supramolecular prodrug vesicle also exhibited excellent stability in aqueous solution for several months.

a)

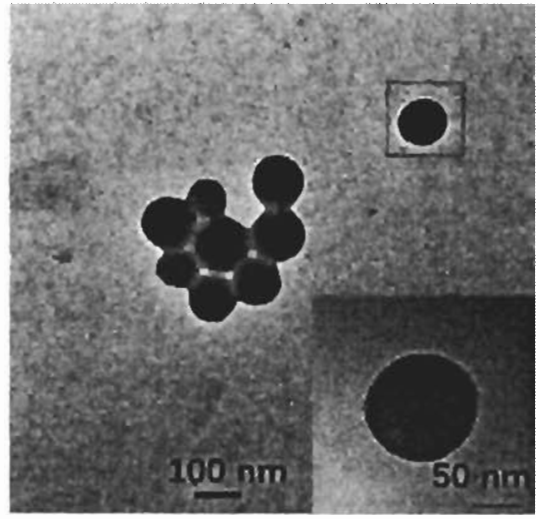

c)

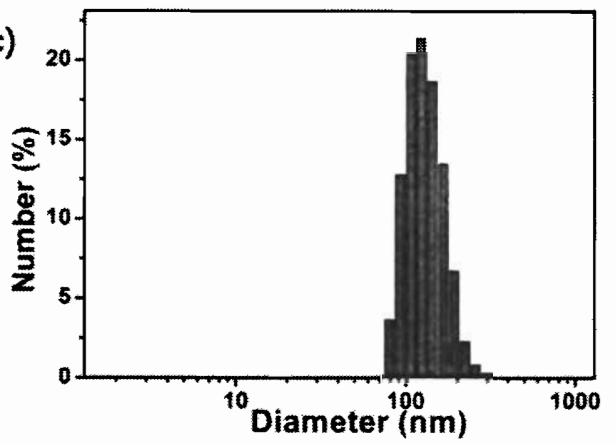

b)

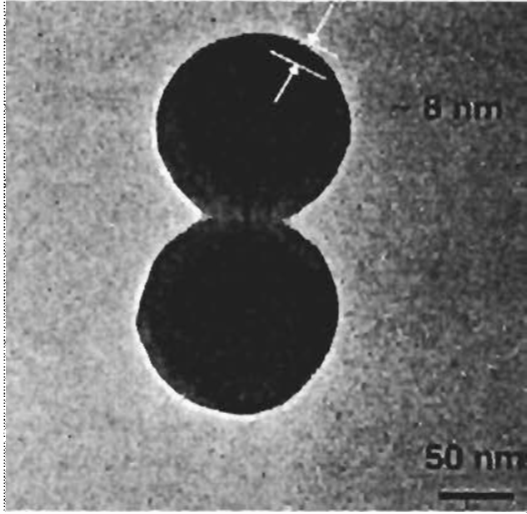

d)

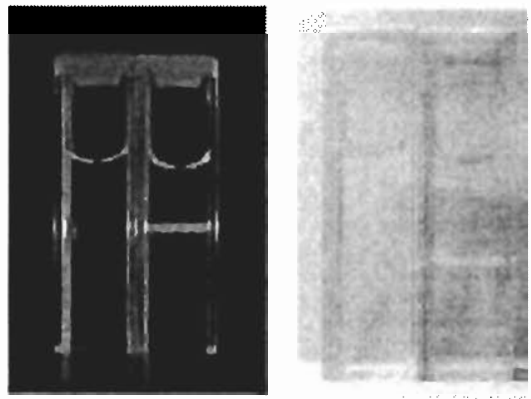

Figure 38 (a) TEM image of the vesicles self-assembled form the prodrug supramolecular amphiphilic vesicles between the H2 and G1, scale bar $=100 \mathrm{~nm}$, insert: enlarge TEM image of the vesicle, scale bar $=50 \mathrm{~nm}$. (b) Enlarge TEM image of the vesicles, the thickness is about $8 \mathrm{~nm}$, scale bar is $50 \mathrm{~nm}$. (c) DLS data of the vesicles self-assembled form the prodrug supramolecular amphiphilic vesicles. (d) Photography showing the Tyndall effect of the prodrug supramolecular amphiphilic vesicles, left: in the dark, right: in presence of light. 


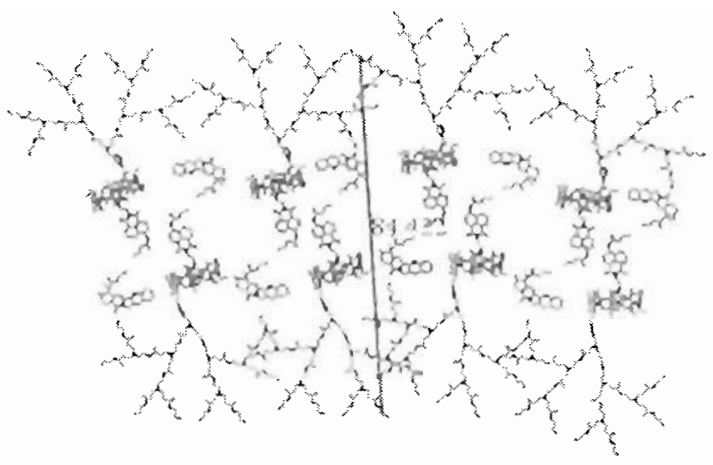

Figure 39 Simulation results of the stacking states of the supramolecular amphiphiles. The thickness of

the wall is about $8.4 \mathrm{~nm}$

\subsubsection{GSH responsive behaviors}

Absorbance and photoluminescence were employed to monitor the spectroscopic changes, upon the addition of the GSH to the phosphate buffer solution (PBS, $\mathrm{pH}=7.4$ ) containing the prodrug supramolecular amphiphilic vesicles. As shown in Figure 41 , a wide absorption band range from $300 \mathrm{~nm}$ to $500 \mathrm{~nm}$ centered at $365 \mathrm{~nm}$ was recorded in absence of the GSH, while a new absorption peak centered at $430 \mathrm{~nm}$ was observed after treatment with the GSH for $2 \mathrm{~h}$ at $37^{\circ} \mathrm{C}$ in PBS buffer $(\mathrm{pH}=7.4)$. For the fluorescence spectra, the emission change of the prodrug supramolecular amphiphilic vesicles was recorded upon time after the addition of GSH $(5.0 \mathrm{mM})$. A rapid emission intensity enhancement at $542 \mathrm{~nm}$ was observed accompanied with a gradually decreasing emission intensity at the $486 \mathrm{~nm}$ over $120 \mathrm{~min}$ (Figure 40). This obvious fluorescence red-shifts endowed the supramolecular amphiphilic vesicles 
ability to monitor the drug release process in real time and helps in the imaging of the cancer cells.

a)

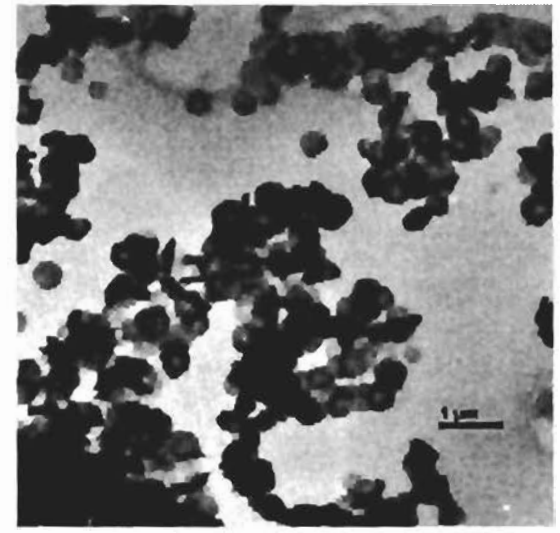

b)

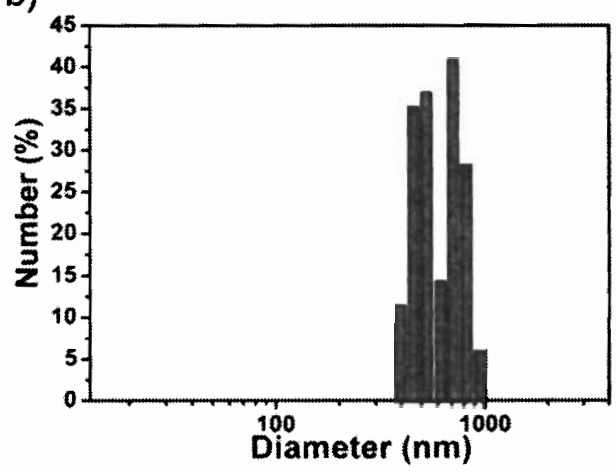

Figure 40 (a) TEM image of the prodrug supramolecular amphiphilic vesicles after treatment with GSH,

scale bar $=1 \mu \mathrm{m}$. (b) DLS data of the prodrug supramolecular amphiphilic vesicles after treatment with the GSH.

Upon treatment with the GSH in the PBS buffer $(\mathrm{pH}=7.4)$ at $37^{\circ} \mathrm{C}$ over $2 \mathrm{~h}$, the solution was characterized by TEM and DLS. Compared with the solution in absence of the GSH, a wide size distribution range from $500 \mathrm{~nm}$ to $1000 \mathrm{~nm}$ was observed in the GSH treated solution, indicating that the vesicles were successfully degraded by the GSH (Figure 40). TEM was also employed to characterize the morphology, the defective capsule like structure was observed with the size of over $500 \mathrm{~nm}$. After disulfide bond cleavage by GSH, the CPT segment was released, resulting of a complex of adamantane linked naphthalimide with dendritic cyclodextrin, and the sphere like structure was also observed, because supramolecular amphiphilic property of the host-guest complex still remained (Figure 40). 

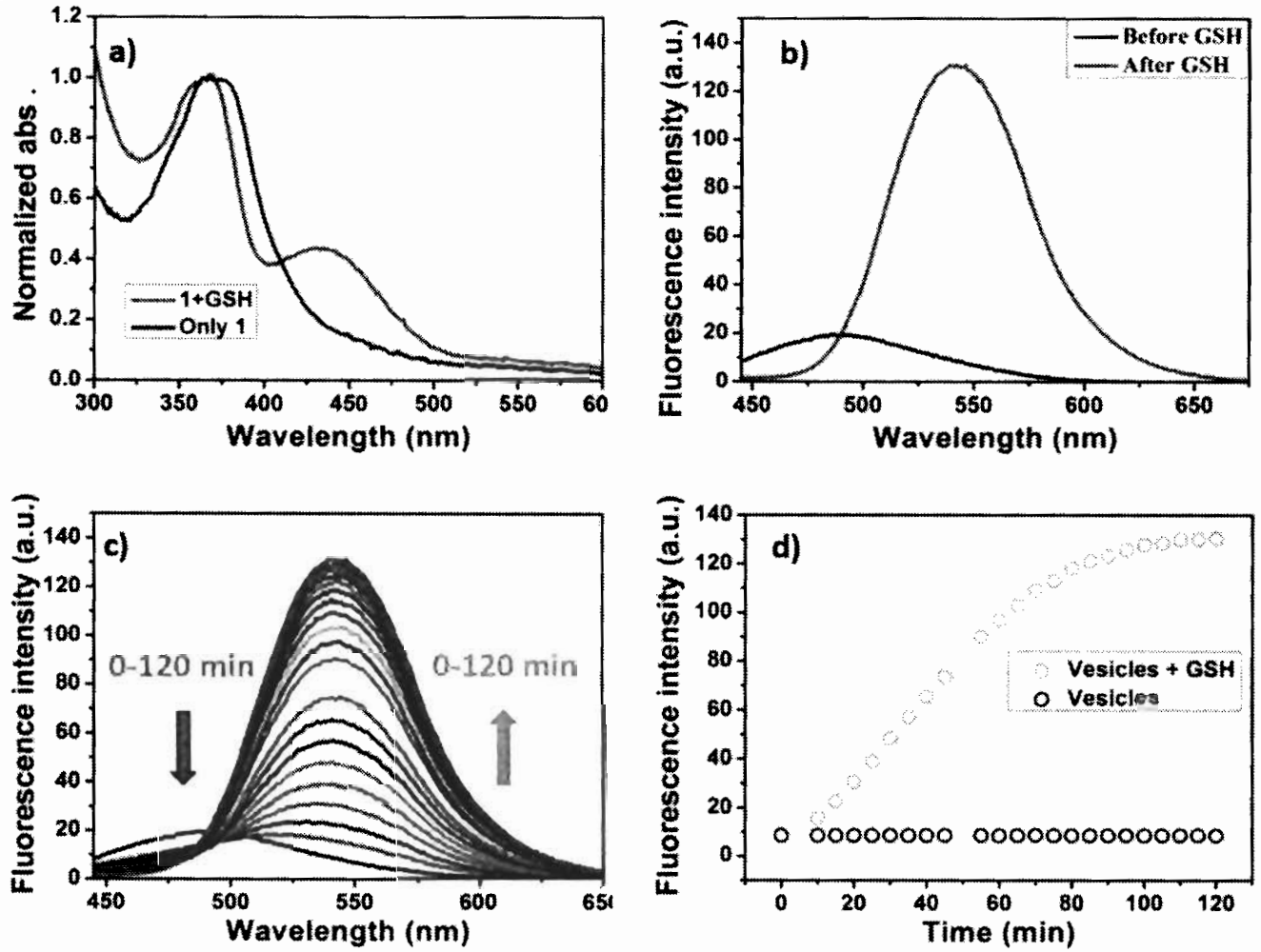

Figure 41 . (a) Absorption and (b) fluorescence spectra of prodrug supramolecular amphiphilic vesicles

$(5.0 \mu \mathrm{M})$ recorded in the presence and absence of $G S H(5.0 \mathrm{mM})$. (c) Time-dependent fluorescence spectral changes observed when prodrug supramolecular amphiphilic vesicles $(5.0 \mu M)$ was treated with GSH (5.0 mM) at $37{ }^{\circ} \mathrm{C}$ in PBS buffer (pH 7.4). 1ex $=430 \mathrm{~nm}$. (d) Time-dependent change in fluorescence intensity at $540 \mathrm{~nm}$.

This GSH triggered release mechanism was presented below after referring to some literatures. Initially, the disulfide bond was cleaved to afford two unstable intermediates in the presence of the GSH. Then adamantane attached fluorophore intermediate product undergoes an intramolecular cyclization to give the unit with the emission color changing from blue to green (Figure 42). The CPT intermediate part also undergoes an intramolecular cyclization to release free CPT drug (Figure 42). 


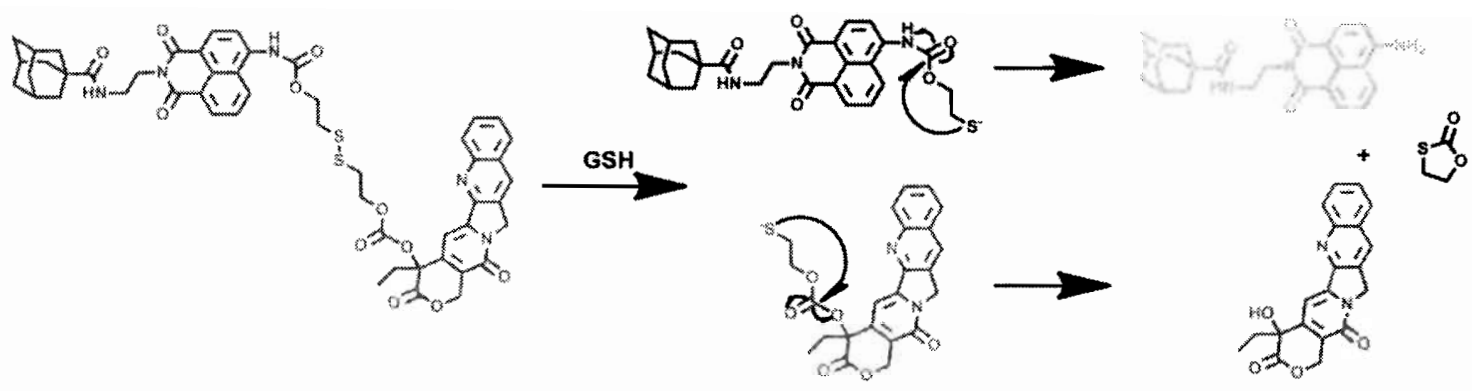

Figure 42 Proposed reaction mechanism of $G 1$ with GSH under physiological conditions.

ESI-MS and reverse-phase HPLC were employed to confirm the release mechanism of GSH induced disulfide cleavage following with intramolecular cyclization. From the HPLC chromatograms results, free CPT molecules exhibited a sharp retention time peak at 16.146 min monitoring with the absorption wavelength of $270 \mathrm{~nm}$, and the adamantane attached pro-CPT exhibited a major peak at $27.1 \mathrm{~min}$. After treatment with GSH in PBS buffer with the $\mathrm{pH} 7.4$, samples were analyzed at different time points for HPLC testaments. As shown in Figure 43, after treatment with GSH for $20 \mathrm{~min}$, a new peak at $16.146 \mathrm{~min}$ was observed, which could be assigned to free CPT. After treatment of the GSH over $200 \mathrm{~min}$, the intensity of the major peak at $27.1 \mathrm{~min}$ decreased with time, while the intensity of the peak at 16.146 min was enhanced. As shown in Figure 43, the ratio of the CPT released reached to $96 \%$, indicating that almost all prodrug was converted to free CPT within $200 \mathrm{~min}$ in presence of GSH. 


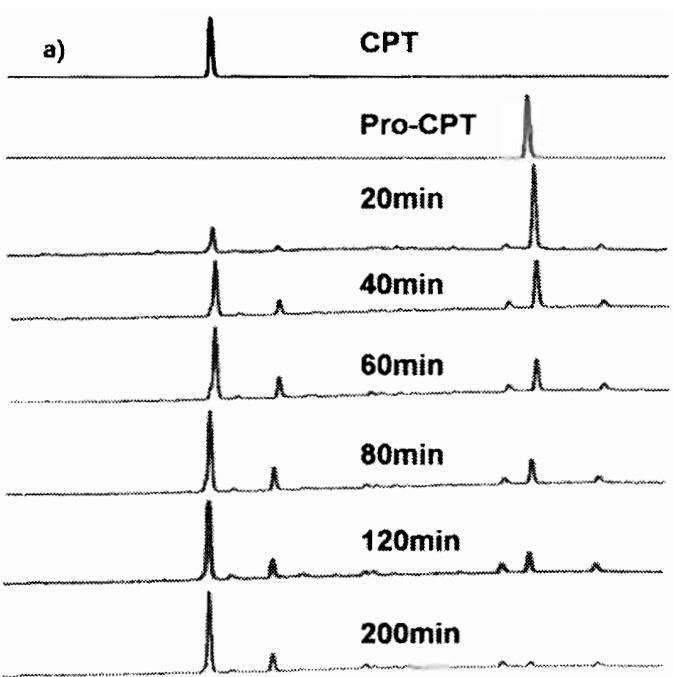

b)

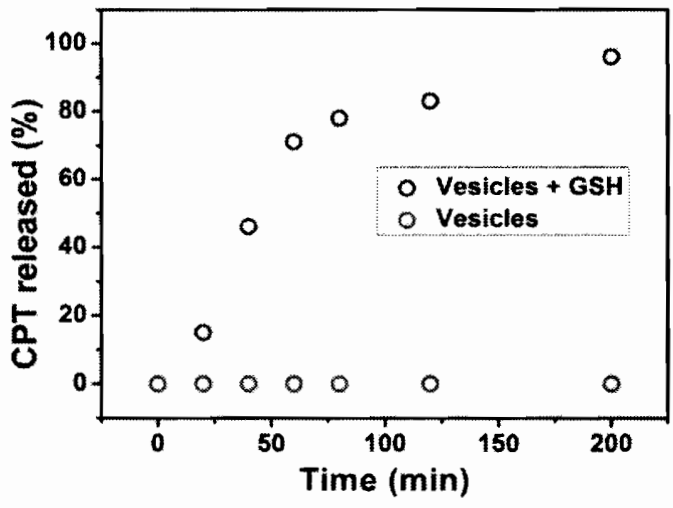

Figure 43 (a) CPT in reverse-phase HPLC chromatograms was detected by UV absorption using $250 \mathrm{~nm}$ as the interrogation wavelength. (b) Time-dependent change in peak intensity at $16.145 \mathrm{~min}$.

As shown in Figure 44, peaks found at 440.43, 545.24 and 972.83 could be assigned to compound 4, CPT, and G1 respectively. From the ESI-MS data, compound 4 and free CPT were detected after reduction in the presence of GSH, indicating that the mechanism proposed above was reasonable. 


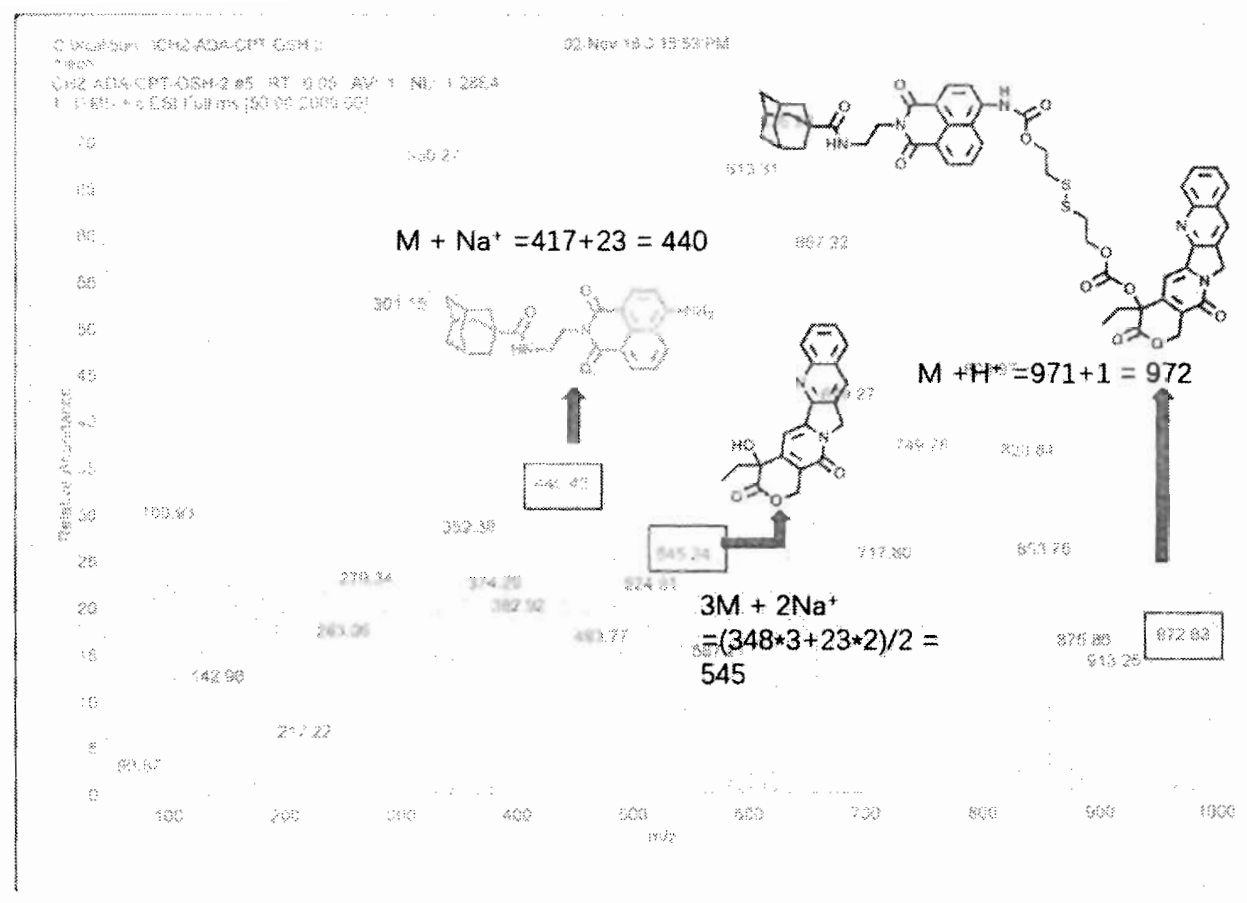

Figure 44 ES/-MS of the prodrug supramolecular amphiphilic vesicles after incubation with the GSH 5 $m M$, in PBS buffer with $\mathrm{pH}=7.4$, at $37^{\circ} \mathrm{C}$ for $1 \mathrm{~h}$

\subsubsection{Cellular uptake study}

To confirm the successfully cellular uptake of the vesicles, confocal laser scanning microscope (CLSM) was employed to detect the cellular fluorescence changes. HeLa cells was incubated with vesicles with the concentration of $2 \mu \mathrm{M}$ for $12 \mathrm{~h}$, followed by fixing for characterization. As shown in Figure 45, strong fluorescence was observed in both blue and green channel, indicating that the vesicles were successfully internalized by the HeLa cells. The obvious green also proved that prodrug can be efficiently cleaved to release the fluorophore inside the cancer cells. 
a) $\mathrm{i}$

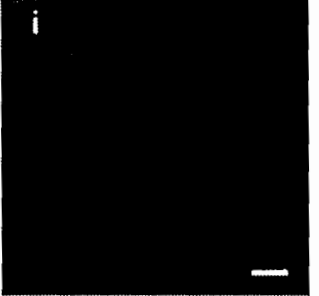

b)

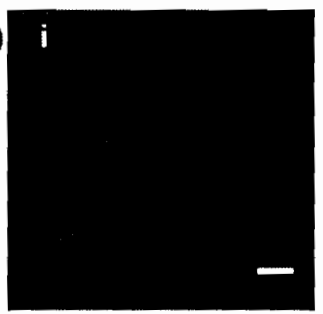

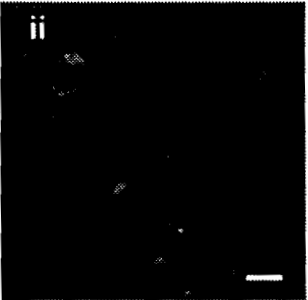

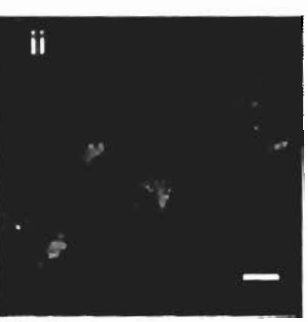

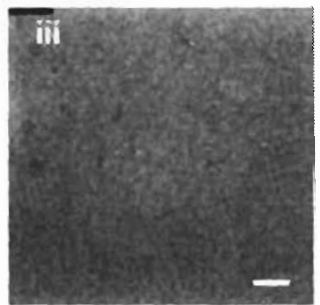

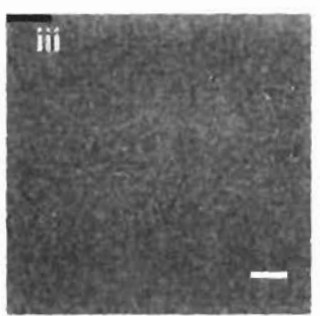

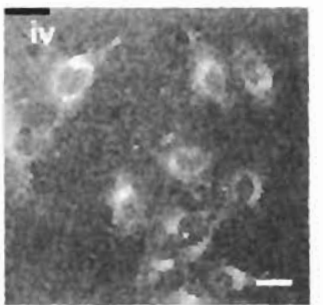

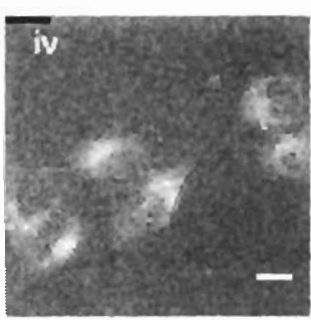

Figure 45 (a) Confocal fluorescence images of HeLa cells: HeLa cells incubated with the vesicles (2 $\mu \mathrm{M})$ for $12 \mathrm{~h}$ : i) blue channel at $450 \pm 35 \mathrm{~mm}$, ii) green channel at $515 \pm 30 \mathrm{~mm}$, iii) bright-field transmissiom image. (iv) Overlap image generated from (i) and (ii), scale bar=20 $\mu \mathrm{m}$. (b) Entarge confocal fluorescence images of HeLa cells. scale bar $=10 \mu \mathrm{m}$.

\subsubsection{Cytotoxicity and in vitro antitumor study}

The cytotoxicity of the dendritic cyclodextrin (H2) was measured by using MTT assay. As shown in Figure 46, H2 exhibited extremely high biocompatibility with negligible cytotoxicity even at very high concentration, indicating that $\mathrm{H} 2$ was suitable for acting as cargo delivery carrier. To identify the cancer therapeutic efficacy, the cancer cells killing ability of the vesicles was also investigated by using MTT assay. When HeLa cells were incubated with free CPT, ADA-CPT (prodrug), and CD-CPT (supramolecular amphiphilic vesicles) for $72 \mathrm{~h}$ respectively, the dose dependency cytotoxicity was also observed. The results of the MTT assay shown in Figure 46, the free CPT displayed similar cytotoxicity as compared to the ADA-CPT at the low concentration and higher cytotoxicity at high concentration, which was reasonable because ADA-CPT was activated under the GSH mediated intramolecular 
cyclization to release free CPT. Time is needed for the reduction by GSH to occur and this is especially noticeable at the high concentrations. The most interesting thing was that CD-CPT exhibited much higher cytotoxicity than the free CPT and ADA-CPT at the high concentration conditions, as well as similar cytotoxicity at the low concentration conditions. The significant cytotoxicity enhancement indicated that the vesicles were preferably internalized by the HeLa cells through endocytosis. As the data presented above, this supramolecular amphiphilic prodrug vesicle exhibited higher cellular uptake efficacy and better therapeutic performance than the free CPT and prodrug itself.
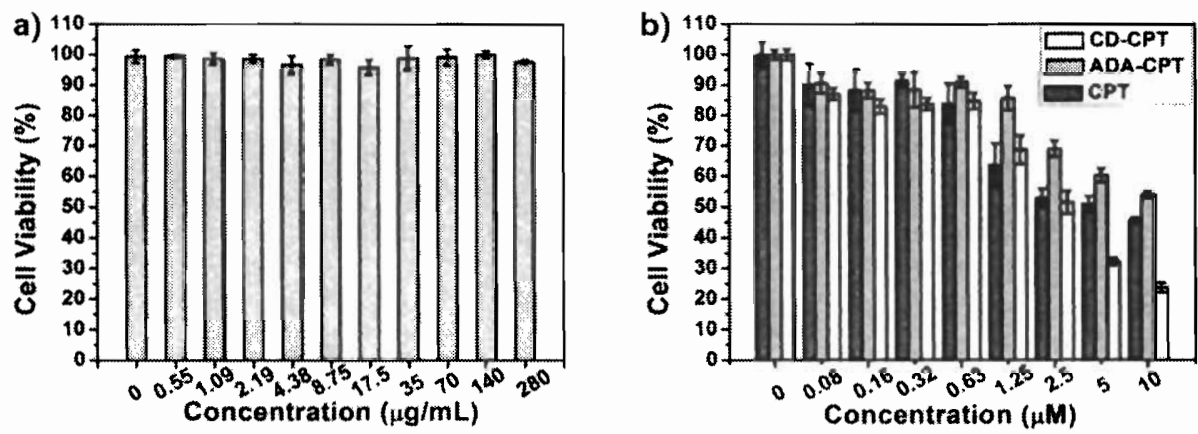

Figure 46 (a) Cell viability of $\mathrm{H} 2$ at different concentrations. The HeLa cells were seeded at $1 \times 104$ cells/well on a 96-well plate. When 70-80\% confluency was reached, the cells were treated with different concentrations of Hy dissolved in DMEM media for $48 \mathrm{~h}$ at $37^{\circ} \mathrm{C}$. After this incubation time, an MTT assay was performed. (b) Cell viability of free CPT, ADA-CPT (prodrug only), and CD-CPT (supramolecular amphiphilic vesicles) at different concentrations. The HeLa cells were incubated for $72 \mathrm{~h} 37^{\circ} \mathrm{C}$.

\subsubsection{SiRNA delivery study}

Due to plenty of free amine groups on the surface of the vesicles, the vesicles inherently were able to be utilized as gene carriers. Critically, free $\mathrm{H} 2$ displayed high 
biocompatibility and negligible toxicity, indicating that $\mathrm{H} 2$ has a great advantage as compared to most of toxicity gene carriers. In this work, the prodrug supramolecular amphiphiles vesicles were also used as siRNA vector for achieving chemo/gene synergetic cancer therapy. The N/P (terminal amines/phosphates) ratio was also optimized by using the electrophoretic mobility experiments in agarose gel. As shown in Figure 47 complete retardation of the siRNA was achieved at the N/P ratio at 25/1. To test the siRNA stability after binding with the vesicles, the siRNA vesicles complexes were incubated with RNase A over $120 \mathrm{~min}$, and samples collected at different time points were analyzed using electrophoresis with $2 \%$ agarose gel.

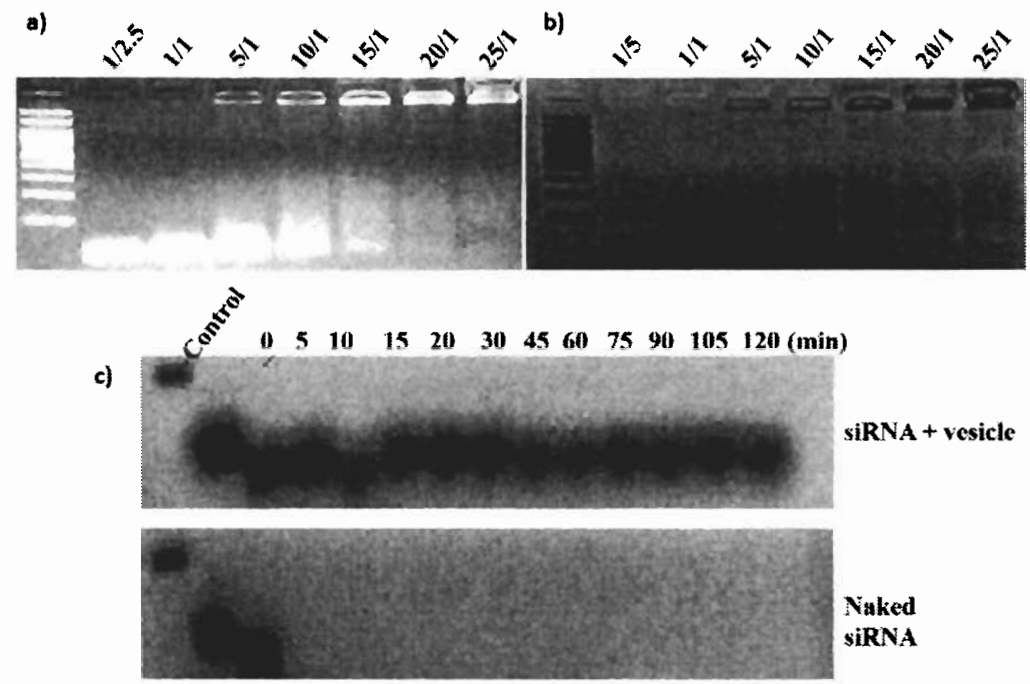

Figure 47 (a) and (b) Gel retardation of 200ng siRNA in agarose ge/ with vesicles at N/P charge ratios ranging from 1:2.5 to 25:1. (c) The resulting siRNA/vesicle complexes protect siRNA from RNase degradation.

As shown in above TEM and DLS data, the supramolecular amphiphilic complex was observed spherical nanostructure with the uniform size about $110 \mathrm{~nm}$. Upon interaction with the siPlK1, the supramolecular amphiphilic vesicles underwent 
structure shrinkage to form small vesicle like structures. The size distribution was determined by the DLS and the size was about $72 \mathrm{~nm}$ with relative narrow distribution, Figure 48. Vesicle like structures with the uniform size about $67 \mathrm{~nm}$ was observed under the TEM, which was in accordance with the results of the DLS data (Figure 48). The curvature of the vesicles was regulated by many factors, including charge distribution, rigid of the molecule, hydrophilic area, and hydrophobic volume. Smaller size indicating the higher curvature of the vesicles and that the size shrinkage was the result of the curvature increment. In this system, the curvature increment could be attributed to the surface charge changes. Because of the abundant amine groups on the surface, the vesicles showed positive surface charge. After interaction with the negative charged siRNA, the positive charge of the vesicles was partly neutralized by the siRNA. The electrostatic repulsion between the supramolecular amphiphilic complexes was decreased with the addition of the siRNA, resulting of the more compact packing between the supramolecular amphiphilic complexes, leading to curvature increment. The hypothesis was proved by the zeta-potential, the vesicles exhibited $+23.6 \mathrm{mV}$ in absence of the siRNA, and $+18 \mathrm{mV}$ in presence of the siRNA (Figure 48). 
a)

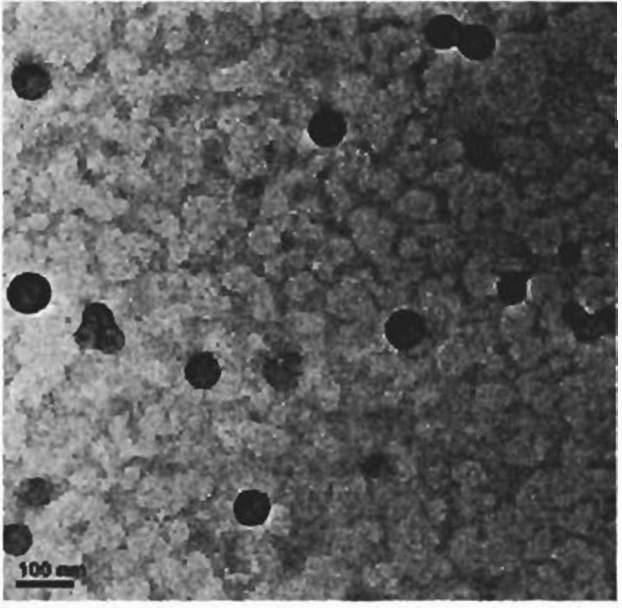

c)

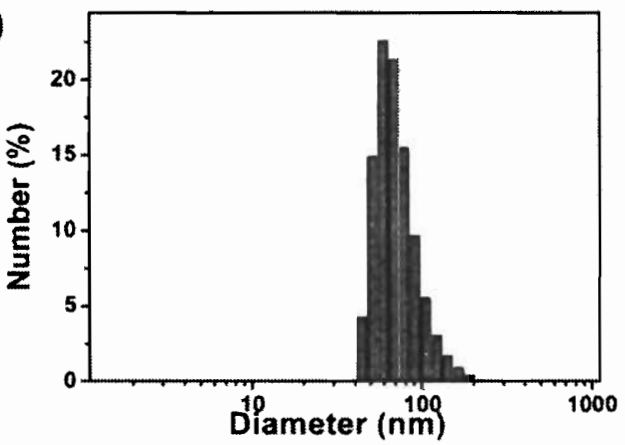

b)

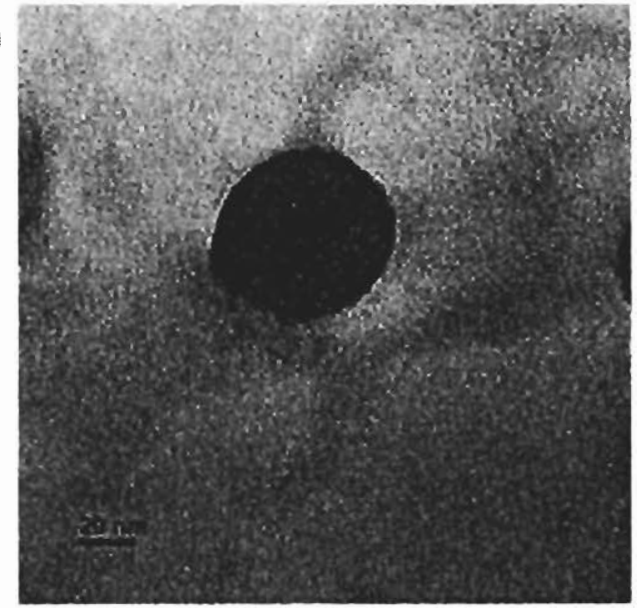

d)

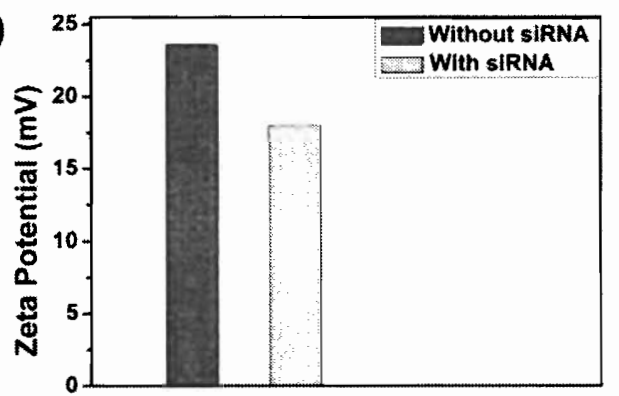

Figure 48 (a) TEM image of the supramolecular amphiphilic vesicles after addition of the siRNA, scale bar $=100 \mathrm{~nm}$. (b) Enlarge TEM image of the supramolecular amphiphilic vesicles after treatment with the siRNA, scale bar $=20 \mathrm{~nm}$. (c) DLS data of the supramolecular amphiphilic vesicles in presence of the siRNA. (d) The zeta potential of the supramolecular amphiphilic vesicles in presence of the siRNA and absence of the SIRNA.

Fluorescence emission spectra was used to investigate the interactions between the supramolecular amphiphilic vesicles with the siRNA, cy3 dye as fluorophore was labeled at the 5'site of the siRNA. The supramolecular amphiphilic vesicles solution exhibited colorless, and cy3-siRNA solution displayed pink color. After successfully formation of the complex, the color of the solution turned to dark orange, Figure 49. Upon irradiation of the UV lamp with the wavelength of $360 \mathrm{~nm}$, the vesicles solution 
exhibited blue color emission, and no emission of the cy3-siRNA was observed under the same condition. The complex displayed near white emission in presence of the UV irradiation, due to FRET occurring between the vesicles and the cy3-siRNA, indicating that cy3-siRNA was successfully bonded with the vesicles. The cy3-siRNA cannot be excited by the UV light with the wavelength of $360 \mathrm{~nm}$, since the main absorption band of the cy3-siRNA was located at around the $550 \mathrm{~nm}$. As shown in Figure 49, the cy3-siRNA had a main absorption band range from $500 \mathrm{~nm}$ to $570 \mathrm{~nm}$, which overlapped very well with the emission of the G2. After excitation with the 360 nm light, a new emission peak of the complex was observed which was assigned to the emission of the cy3, indicating efficient FRET of the complex.

a)
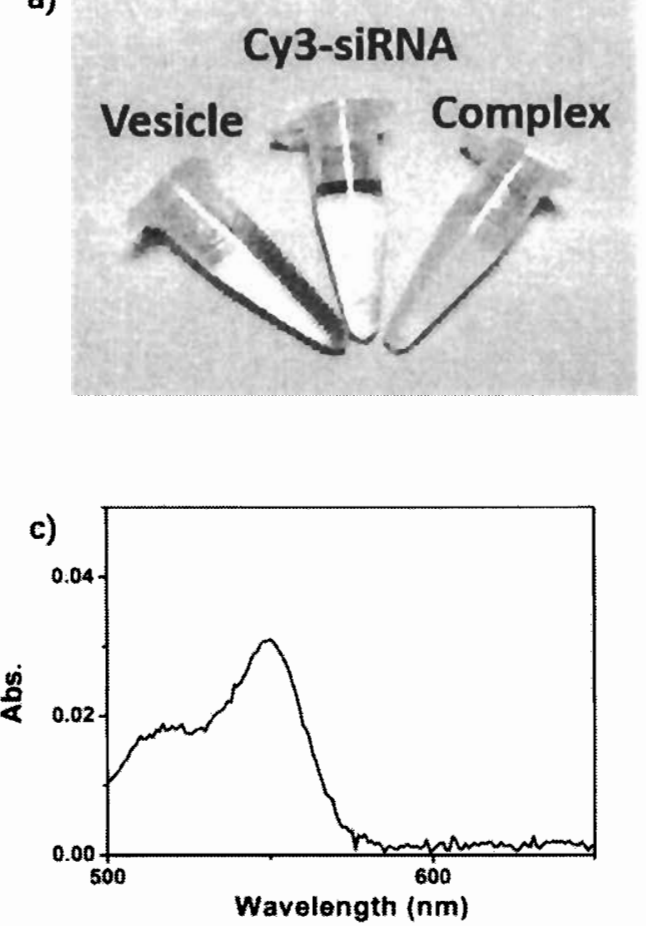

b)

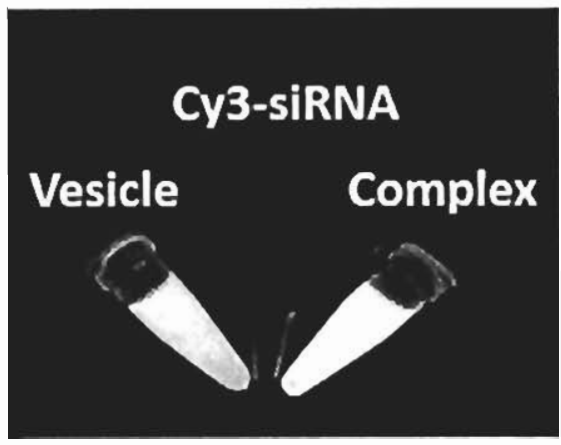

d)

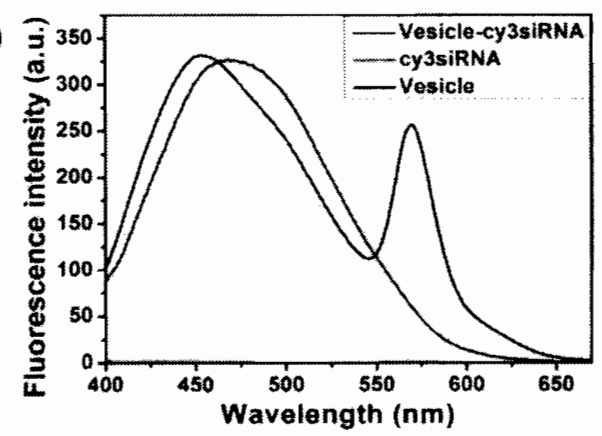

Figure 49 (a) Photography of the supramolecular vesicles solution, cy3-siRNA solution, and vesicles-cy3-siRNA complex solution. (b) The photography of the above solution under the irradiation of the $360 \mathrm{~nm}$ light. (c) UV-absorption spectrum of the cy3-siRNA. (d) Emission spectrum of the cy3-siRNA 
(red curve), supramolecular vesicles (blue curve), and vesicles-cy3-siRNA complex solution (black curve)

the excitation wavelength is $360 \mathrm{~mm}$

The successfully delivery of siRNA in cellular level was also confirmed by the CLSM. HeLa cells were incubated with vesicles cy3-siRNA complex with the concentration of $2 \mu \mathrm{M}$ and incubated for $12 \mathrm{~h}$, followed by fixation using $4 \%$ formaldehyde. As shown in Figure 50, strong blue fluorescence emission was observed and assigned to G1, while strong green fluorescence emission was observed and assigned to compound 3. After excitation with the $561 \mathrm{~nm}$ laser, obvious red fluorescence emission was observed, indicating that the supramolecular amphiphilic vesicles successfully delivered the siRNA into the HeLa cells. The results of the CLSM proved that the supramolecular vesicles were able utilized as gene vector to deliver nucleotides for gene therapy.

a)

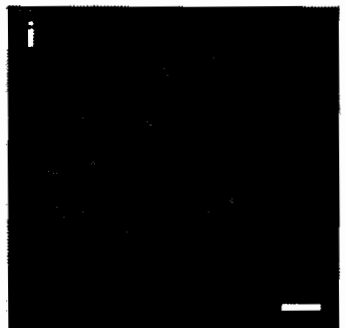

b)

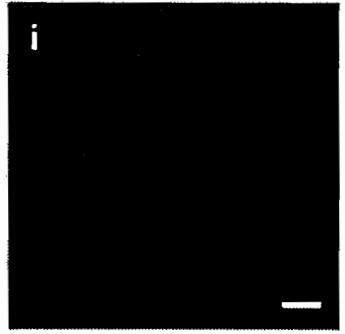

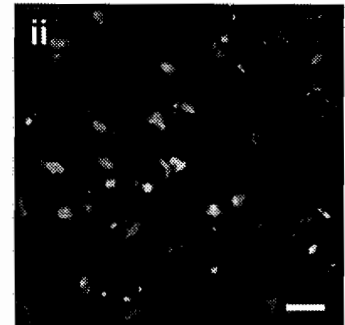

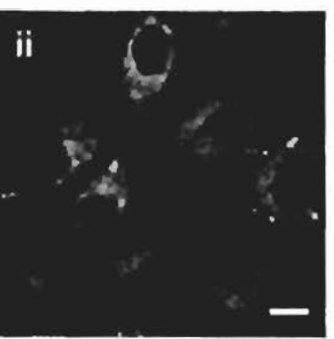

iii
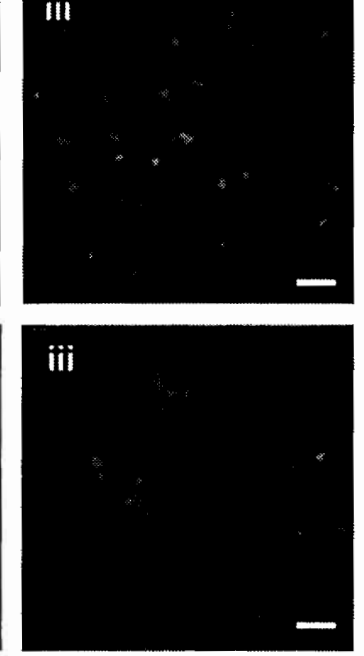
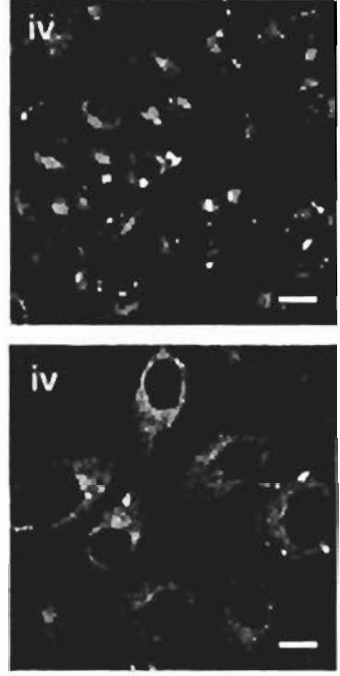

Figure 50 (a) Confocal fluorescence images of HeLa cells: Hela cells incubated with the vesicles-cy3-siRNA complexes ( $2 \mu \mathrm{M})$ for $12 \mathrm{~h}$ : i) blue channel at $450 \pm 35 \mathrm{~nm}$, ii) green channel at 515 
$\pm 30 \mathrm{~nm}$, iii) cy3 channel, (iv) Overlap image generated from (i) and (ii), and (iii), scale bar= 20 um. (b)

Enlarge confocal fluorescence images of HeLa cells. scale bar $=10 \mathrm{~mm}$

The cytotoxicity of the vesicles after introduced siRNA was also investigated by the MTT assay. The siPlk1 was chosen as therapeutic active agents, while siNC with non-active sequences was employed as negative control. After incubated with the HeLa cells for $72 \mathrm{~h}$, MTT assay was employed to characterize the cell viability of the HeLa cells. As shown in Figure 51, prodrug supramolecular amphiphilic vesicles suppressed the cell viability to about $22 \%$ at the concentration of $10 \mu \mathrm{M}$. CPT-CD-siPIK1 (supramolecular amphiphilic vesicles loaded siPIK1) exhibited obvious cell viability inhibition enhancement up to $11 \%$, indicating successfully chemo/gene synergetic therapy. As for the negative control, CPT-CD-siNC also displayed slight cell viability suppression improvement, which may be attributed to the size and surface changes. 


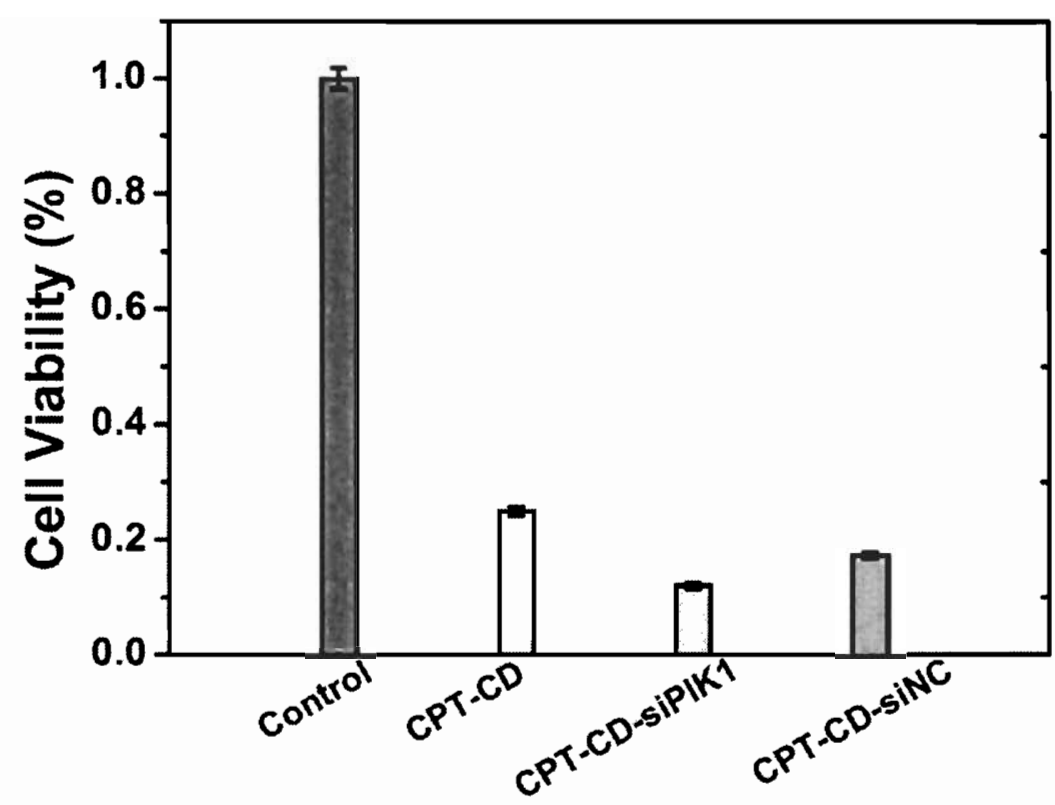

Figure 51 Cell viability of CPT-CD (supramolecular amphiphilic vesicles), CPT-CD-siPIK1 complex, and CPT-CD-siNC complex with the concentration of $10 \mu \mathrm{M}$ and SiRNA concentration of $100 \mathrm{nM}$. The HeLa cells were incubated for $72 \mathrm{~h} 37^{\circ} \mathrm{C}$.

\subsection{Conclusion}

In summary, using host-guest interaction concept as the basis for construction of supramolecular amphiphiles, we successfully designed and fabricated a GSH responsive dendritic supramolecular theranostic prodrug vesicle. By taking advantage of the supramolecular strategy, construction of the prodrug assembly was much easier in comparison to conventional synthetic approaches. The fluorophore segment of the prodrug underwent significant red-shift after disulfide cleavage followed by intramolecular cyclization. At the same time, free drug was released, which was used to image the cancer cells in real time and for therapeutic purposes. The supramolecular prodrug vesicle exhibited excellent water solubility as well as stability 
and displayed higher therapeutic performance than the original anticancer drug for in vitro studies. Furthermore, the abundance of free amine groups at the terminal sites of the dendrimer provides binding sites, enabling the supramolecular amphiphilic vesicle to be employed as a siRNA delivery vector for chemo/gene combination therapy. Fluorescence studies, TEM, and DLS results confirmed the efficient siRNA loaded by the supramolecular amphiphiles. Cell studies also proved the siRNA was transported into the cells and improved the cancer therapy efficacy. Here, we developed a prodrug that was formed into supramolecular amphiphiles via host-guest interaction and utilized the assembly as gene vector to achieve dual therapy that results in synergistic effect, which provide a new strategy to deliver both prodrug and therapeutic gene, showing promising applications in cancer treatments. Further studies are currently ongoing. 


\section{Chapter 4: Bio-degradable Bola Type Supramolecular AIE Vesicle for delivery of siRNA}

\subsection{Introduction}

In recent years, gene therapy including pDNA, mRNA, siRNA has attracted lots of attention due to its inherent advantages. Due to the ability to efficiently silence the disease-related genes, siRNA (small interfering RNA) has drawn much attention in treatment in oncology. However, lack of the safe and effective delivery vectors restricts the application of siRNA for gene therapy, because siRNAs are rapidly degraded in body fluid. Viral vectors have been developed with highly transfection efficacy, but their potential inflammatory immune responses, and gene control effect result in serious safety risks. In the past decades, non-viral alternatives have been developed including cationic lipids and polymers. Both of them possess existing the drawbacks, the cationic lipids are limited to be used in in vivo applications due to highly positive charge density, resulting in high cytotoxicity and polymers was undesirable due to undefined structural composition. Development of the non-viral vectors without the shortcomings of the polymers and cationic lipids has attracted abundance attention in gene therapy field.

Aggregation-induced emission (AIE) has attracted lots of attention in recent years, because it can overcome the shortage of the ACQ (aggregation-caused quenching) ${ }^{209-}$ 217. At high concentrations, common fluorescence dyes often experience quenching or 
weakening in fluorescence intensity, which thus limits the practical applications of dye, especially in field of bio-imaging. On the other hand, aggregates of AIE type fluorophores show strong fluorescence because of its restriction in intramolecular rotations. AIE thus provides a new platform for exploration of photostable and fluorescence light-up nanoaggregates for bio-detection and imaging ${ }^{57,218-225}$.

In our previous work, we reported supramolecular prodrug vesicle and used the vesicle to deliver therapeutic siRNA for combination cancer therapy. Based on the previous study on using of supramolecular amphiphile for siRNA delivery, we questioned how the structure of the supramolecular amphiphiles could influence the siRNA delivery efficacy. Herrin, we developed a bola type TPE supramolecular vesicle for siRNA delivery. The TPE not only severed as hydrophobic segment but also employed as AIE fluorophore for real time monitoring of celluar uptake. Dendritic cyclodextrin acted as hydrophilic segment and siRNA binding receptor. The supramolecular complex self-assembled to form vesicle in aqueous solution and can be degraded because of the disulfide linkage. The bio-degradable bola type TPE supramolecular vesicle was used to deliver the siRNA into cancer cells (Scheme 6). 
a)
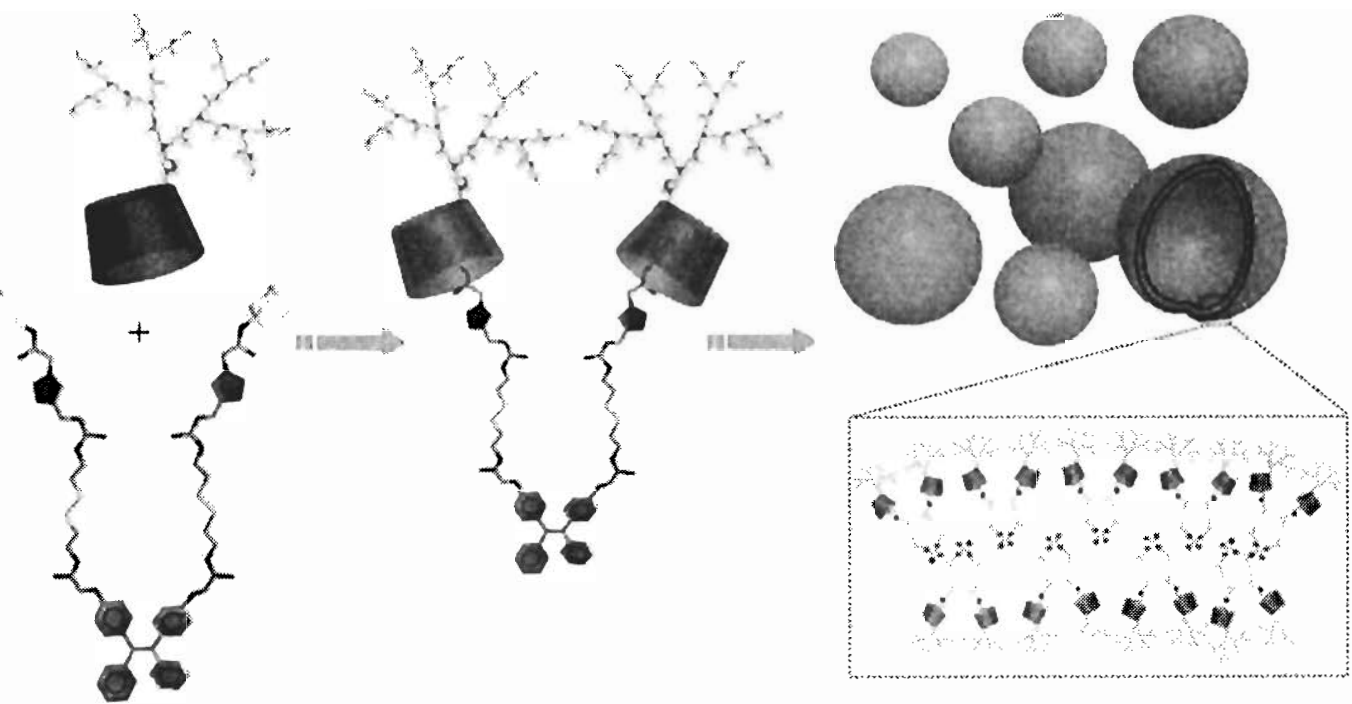

b)

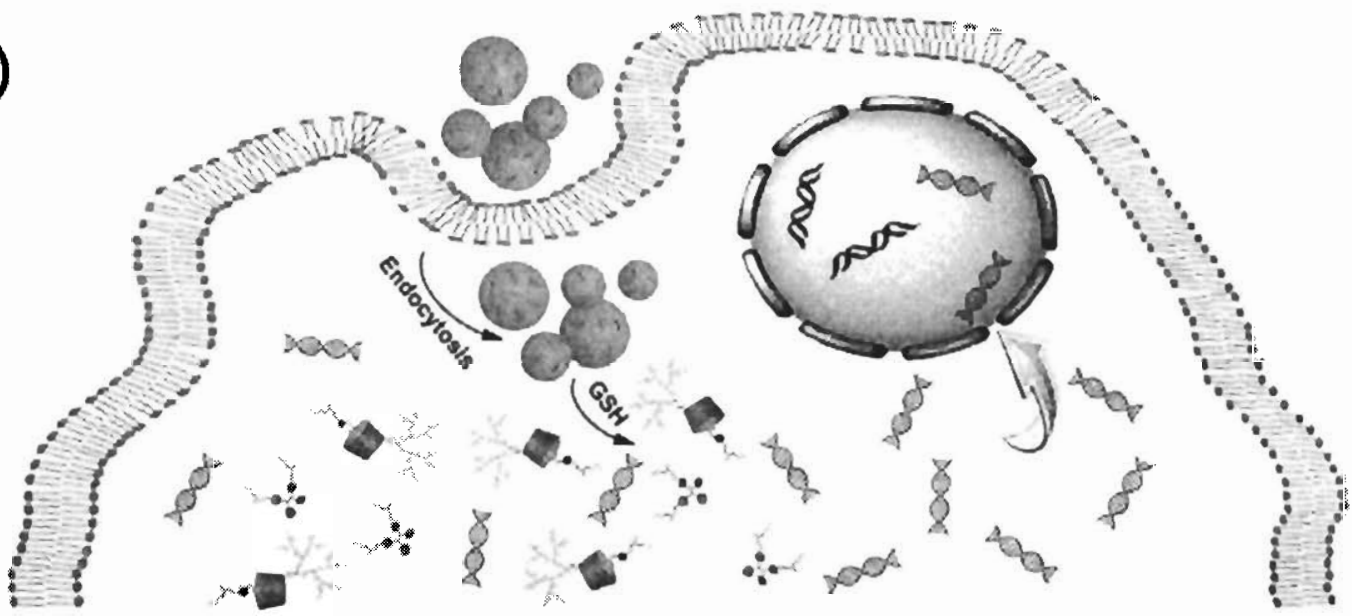

Scheme 6 (a) Schematic illustration of the fabrication of the bola type TPE supramolecular vesicle. (b)

Schematic illustration of the siRNA release process.

\subsection{Materials and Characterizations}

THF and toluene were refluxed with sodium overnight before used. DCM and was refluxed with $\mathrm{CaH}_{2}$ overnight before used. DMF was refluxed with $\mathrm{CaH}_{2}$ overnight and distilled under the reduced pressure before used. Ethanol was refluxed with magnesium and iodine overnight and distilled before used. SiPIK1 with the sequence of (sense strand, 5-UGAAGAAGAU-CACCCUCCUUAdTdT-3; antisense strand, 
5-UAAGGAGGGUGAUCUUCUUCAdTdT-3) and scrambled siRNA (siNonsense) with the sequence of (sense strand, 5-UUCUCCGAACGUGUCACGUdTdT-3; antisense strand, 5-ACGUGACACGUUCGGAGAAdTdT-3) were supplied by Su Zhou Ribo Life Science Co. Ltd. (Suzhou, China). Cy3 labeled siRNA with the sequence of (sense strand, 5'-UUCUCCGAACGUGUCACGUdTdT-'3; antisense strand, 5'-ACGUGACACGUUCGGAGAAdTdT-3', labeled with cy3 at 5 position) was supplied from shanghai Genepharma (Shanghai China). Propargyl alcohol, 18-crown-6, Cystamine dihydrochloride, 1,1'-Carbonyldiimidazole, potassium carbonate, bromoacetyl chloride, 4-Hydroxybenzophenone, amantadine hydrochloride, pyridine, zinc powder, sodiumiodide, 2-azidoacetic acid, 4-dimethylaminopyridine (DMAP), 1-ethyl-3-(3-dimethylaminopropyl) carbodiimide (EDC), ethylenediamine, 1-Hydroxybenzotriazole hydrate (HOBT), iodo(triethyl phosphite)copper(I), and L-ascorbic acid sodium salt were obtained from Sigma Aldrich and were used without further purification. Titanium tetrachloride was obtained from the Merck and used without purification. The cyclodextrin was a gift given by my friend. Dendrimer $\mathrm{H} 1$ and propargyl cystamine were synthesized according the literature reported procedures $^{226}$.

${ }^{1} \mathrm{H}$ and ${ }^{13} \mathrm{C}$ nuclear magnetic resonance (NMR) spectrum was measured on a Bruker BBFO-4.00 spectrometer using deuterated chloroform $\left(\mathrm{CDCl}_{3}\right)$, deuterated tetrahydrofuran (Tetrahydrofuran- $\left.\mathrm{d}_{8}\right)$ and deuterated dimethyl sulphoxide (DMSO- $\mathrm{d}_{6}$ ) as the solvents. The electronic spray ionization (ESI) mass spectra were recorded on a ThermoFinnigan LCQ quadrupole ion trap mass spectrometer. High-resolution mass 
spectrometry (HR-MS) was performed on a Waters Q-tof Premier MS spectrometer. Transmission electron microscopy (TEM) images were collected on JEM-1400 (JEOL) at $100 \mathrm{kV}$. The fluorescence emission spectra were recorded on a Shimadzu RF-5301pc fluorescence spectrophotometer. UV-vis spectra were recorded from Shimadzu UV-3600 spectrophotometer. Zeta-potential values were determined by a Malvern Instruments Zetasizer Nano-S at $25^{\circ} \mathrm{C}$. DLS size distributions were measured on a Nanobrook 90Plus particle size analyzer. MTT was recorded on a Tecan Infinite M200. CLSM was recorded on a Carl Zeiss LSM 800. Gel retardation assay was recorded on Horizontal Electrophoresis systems, Bio-Rad, Singapore.

\subsubsection{Synthesis of the compounds}

\subsubsection{Synthesis of compound 2}

Compound 3 (300 mg, $1.28 \mathrm{mmol})$ was dissolved in dry DCM $(15 \mathrm{~mL})$, followed with addition of the TEA (194 $\mathrm{mg}, 1.92 \mathrm{mmol})$. The mixture was allowed to stir in an ice bath. The bromoacetyl chloride $(300 \mathrm{mg}, 1.92 \mathrm{mmol})$ was dissolved in dry DCM and added dropwise into the mixture over $30 \mathrm{~min}$. The reaction was allowed to warm up to room temperature and stirred overnight. The solution was washed with the water for three times and the organic layers were collected, followed by drying over anhydrous sodium sulfate. The organic solvent was removed under the reduced pressure to give the crude product. The crude product was purified by the silica gel chromatography with the eluent EtOAc:Hexane $=1: 1$ to give the product as colorless oil. The oil was recrystallized from the DCM with Hexane to afford the product as mica solid (270 mg, 76.5\%). 'H NMR (400 MHz, $\mathrm{CDCl}_{3}, 298 \mathrm{~K}$ ): $\delta 7.03$ (s, 1H), 5.33 
$(\mathrm{s}, 1 \mathrm{H}), 4.72(\mathrm{~d}, 2 \mathrm{H}), 4.10(\mathrm{~s}, 1 \mathrm{H}), 3.67(\mathrm{~m}, 2 \mathrm{H}), 3.56(\mathrm{dd}, 2 \mathrm{H}), 2.86(\mathrm{~m} .4 \mathrm{H}), 2.50(\mathrm{t}$, 1H). ${ }^{13} \mathrm{C} \mathrm{NMR}\left(100 \mathrm{MHz}, \mathrm{CDCl}_{3}, 298 \mathrm{~K}\right): \delta 166.0,155.1,78.1,74.8,52.7,42.6,40.0$, 38.9, 37.6, 29.1. HRMS (TOF) $\mathrm{m} / \mathrm{z}[\mathrm{M}+\mathrm{H}]^{+}$, calcd for $\mathrm{C}_{10} \mathrm{H}_{15} \mathrm{BrN}_{2} \mathrm{O}_{3} \mathrm{~S}_{2}, 354.9786$; found, 354.9795 .

\subsubsection{Synthesis of compound 1}

TPE-2OH (154mg, $0.423 \mathrm{mmol})$ and compound $2(460 \mathrm{mg}, 1.3 \mathrm{mmol})$ were dissolved in dry acetone $(20 \mathrm{~mL})$. Potassium carbonate $(230 \mathrm{mg}, 1.66 \mathrm{mmol})$ was added into the mixture. Sodiumiodide was added with catalytic amount, accompanied with 18-crown-6 as phase transfer catalyst. The mixture was allowed to reflux under the protection of the $\mathrm{N}_{2}$ for $6 \mathrm{~h}$. TLC was used to monitor the reaction with the ratio of EtOAc:Hexane $=2: 1$. After cooling down to room temperature, the mixture was filtered and the filtration was collected. The solvent was removed under the reduced pressure. The crude product was washed with water for three times and dried over anhydrous sodium sulfate. The crude product was then purified with silica gel chromatography. The first component was separated by the eluent with the ratio of EtOAc:Hexane $=1: 1$ to afford the Z-form product $141 \mathrm{mg}$. Then the ratio was changed to EtOAc:Hexane $=2.5: 1$ to give the second component as E-form $166 \mathrm{mg}$. The total weight of the Z- and E-form was $307 \mathrm{mg}$, with the yield $79.5 \%$. ${ }^{1} \mathrm{H}$ NMR $\left(400 \mathrm{MHz}, \mathrm{CDCl}_{3}, 298 \mathrm{~K}\right): \delta 7.09(\mathrm{~m}, 6 \mathrm{H}), 6.99(\mathrm{dd}, 10 \mathrm{H}), 6.68(\mathrm{~d}, 4 \mathrm{H}), 5.48(\mathrm{~s}, 2 \mathrm{H})$, $3.67(\mathrm{~m}, 2 \mathrm{H}), 4.67(\mathrm{~d}, 4 \mathrm{H}), 4.44(\mathrm{~d}, 4 \mathrm{H}), 3.68(\mathrm{q}, 4 \mathrm{H}), 3.52(\mathrm{dd}, 4 \mathrm{H}), 2.83(\mathrm{~m}, 8 \mathrm{H})$, $2.45(\mathrm{t}, 2 \mathrm{H}) .{ }^{13} \mathrm{C} \mathrm{NMR}\left(100 \mathrm{MHz}, \mathrm{CDCl}_{3}, 298 \mathrm{~K}\right): \delta=168.51,155.48,143.77,139.79$, $137.72,132.77,131.29,127.93,126.52,113.86,74.72,67.17,52.59,39.88,38.39$, 
37.92, 37.36, 29.70. HRMS (TOF) $\mathrm{m} / \mathrm{z}[\mathrm{M}+\mathrm{H}]^{+}$, calcd for $\mathrm{C}_{46} \mathrm{H}_{48} \mathrm{~N}_{4} \mathrm{O}_{8} \mathrm{~S}_{4}, 913.2433$; found, 913.2407.

\subsubsection{Synthesis of azide adamantane}

Amantadine Hydrochloride (2.24 g, $12 \mathrm{mmol}$ ) was dissolved in dry THF (40 mL), and pyridine (950 $\mathrm{mg}, 12 \mathrm{mmol}$ ) was also added. The mixture was allowed to stir for 3 h. 2-Azidoacetic acid (1 g, $10 \mathrm{mmol})$, EDC (2.3 g, $12 \mathrm{mmol})$, HOBT (675 mg, 5 mmol), and DMAP (611 $\mathrm{mg}, 5 \mathrm{mmol})$ were added into the mixture, and the resultant solution was stirred overnight at room temperature. TLC was used to monitor the reaction. The solution was diluted with DCM and washed with dilute hydrochloric acid, saturated sodium bicarbonate, and water for three times. The organic phase was separated and dried against anhydrous sodium sulfate. The organic solvent was removed under the reduced pressure to afford the pure product as white crystalline solid , (1.55g, 66\%). ${ }^{1} \mathrm{H}$ NMR (400 MHz, $\left.\mathrm{CDCl}_{3}, 298 \mathrm{~K}\right): \delta 5.90(\mathrm{~s}, 1 \mathrm{H}), 3.86$ (s, 2H), $2.09(\mathrm{~s}, 3 \mathrm{H}), 2.01(\mathrm{~d}, 6 \mathrm{H}), 1.68(\mathrm{~d}, 6 \mathrm{H}) .{ }^{13} \mathrm{C} \mathrm{NMR}\left(100 \mathrm{MHz}, \mathrm{CDCl}_{3}, 298 \mathrm{~K}\right): \quad \delta$ $=164.67,52.66,41.44,36.22,29.38,$. HRMS $\left(\right.$ TOF) $\mathrm{m} / \mathrm{z}[\mathrm{M}+\mathrm{H}]^{+}$, calcd for $\mathrm{C}_{12} \mathrm{H}_{18} \mathrm{~N}_{4} \mathrm{O}, 235.1159$; found, 235.1549 .

\subsubsection{Synthesis of compound $G$ ?}

Compound 1 (200 mg, $0.22 \mathrm{mmol})$ and azide adamantane $(155 \mathrm{mg}, 0.66 \mathrm{mmol})$ were dissolved in dry DMF (10 mL). Iodo(triethyl phosphite)copper(I) (160 mg, 0.44 mmol) was added into the mixture under the protection of the nitrogen. The solution was stirred under the nitrogen protection at $50{ }^{\circ} \mathrm{C}$ for $48 \mathrm{~h}$. After cooling to room temperature, the DMF was removed under the reduced pressure, and the obtained 
residue was dissolved in EtOAc and washed with water for 3 times. The organic phase was collected and dried against sodium sulfate. The solvent was removed under the reduced pressure to give the crude product and followed with purification with silica gel chromatography. Firstly, the impurities were was removed by using the EtOAc as eluent, and then the eluent was change to the EtOAc:MeOH=95:5 to give the product as beige solid, (162 mg, 52.9\%). ${ }^{\mathrm{l}} \mathrm{H}$ NMR (400 MHz, $\left.\mathrm{CDCl}_{3}, 298 \mathrm{~K}\right): \delta 7.78(\mathrm{~s}, 2 \mathrm{H})$, $6.90 \sim 7.15(\mathrm{~m}, 16 \mathrm{H}), 6.64(\mathrm{~m}, 4 \mathrm{H}), 5.63(\mathrm{~s}, 2 \mathrm{H}), 5.21(\mathrm{~d}, 4 \mathrm{H}), 4.93(\mathrm{~m}, 4 \mathrm{H}), 4.44(\mathrm{~d}$, 4H), $4.15(\mathrm{~m}, 8 \mathrm{H}), 3.63(\mathrm{~m}, 4 \mathrm{H}), 3.48(\mathrm{~m}, 4 \mathrm{H}), 3.00(\mathrm{~m}, 4 \mathrm{H}), 2.81(\mathrm{~m}, 4 \mathrm{H}), 2.07(\mathrm{~s}$, $6 \mathrm{H}), 1.95(\mathrm{~d}, 12 \mathrm{H}), 1.66(\mathrm{~s}, 12 \mathrm{H}) .{ }^{13} \mathrm{C} \mathrm{NMR}\left(100 \mathrm{MHz}, \mathrm{CDCl}_{3}, 298 \mathrm{~K}\right): \delta=168.53$, $163.63,156.12,155.51,143.77,139.73,137.81,132.75,131.28,127.81,126.50$, $125.33,113.86,67.11,64.02,58.02,53.64,52.85,41.79,41.29,39.79,37.92,35.47$, 30.42, 29.87, 28.34, 16.06. HRMS (TOF) $\mathrm{m} / \mathrm{z}[\mathrm{M}+\mathrm{H}]^{+}$, calcd for $\mathrm{C}_{70} \mathrm{H}_{84} \mathrm{~N}_{12} \mathrm{O}_{10} \mathrm{~S}_{4}$, 1381.5395; found, 1381.5336.

\subsubsection{Preparation of vesicles and siRNA loading vesicles}

TPE-2Ada was dissolved in DMSO to prepare a stock solution with the concentration of $2 \mathrm{mM}$. The dendritic cyclodextrin was dissolved in DI water to obtain a stock solution with the concentration of $2 \mathrm{mM}$. The stock solution of the host molecule was diluted 20 folds to $100 \mu \mathrm{M}$. Upon sonication, $25 \mu \mathrm{L}$ guest stock solution was slowly added into the above solution $(975 \mu \mathrm{L})$ over 5 min to obtain a cloudy solution. The solution was allowed to stand for overnight at room temperature for sufficient assembled, following dialysis against water for $2 \mathrm{~h}$ to remove trace DMSO. The obtained solution was stocked at the $4{ }^{\circ} \mathrm{C}$. To preparation of the siRNA loading 
vesicles, the siPlK1 was added into the fresh prepared vesicles solution, followed by shaking to mix well, and allowed to stay at $37^{\circ} \mathrm{C}$ over $30 \mathrm{~min}$ for sufficient binding.

\subsubsection{Gel retardation assay and siRNA/vesicles complex protection assay}

For the gel retardation assay, siRNAl and vesicles with proper concentration was prepared. For the each tube, $200 \mathrm{ng}$ of siRNA was added with according amount of polymer base on $\mathrm{N} / \mathrm{P}$ ratio of $1 / 2.5,1 / 1,5 / 1,10 / 1,15 / 1,20 / 1$ and 10/1 respectively. The final volume of each tube was inade up to $10 \mu \mathrm{L}$ with Nuclease Free $\mathrm{H}_{2} \mathrm{O}$. The samples were incubated at $37^{\circ} \mathrm{C}$ for $30 \mathrm{~min}$ before running on $1.2 \%$ agarose gel. Bio-Rad Gel-Doc was used for the observation of gel image.

For the siRNA/vesicles complex protection assay, incubate siRNA and vesicles at $37^{\circ} \mathrm{C}$ for $30 \mathrm{~min}$. Add in RNase A with final concentration of $0.01 \mu \mathrm{g} / \mu \mathrm{L}$, withdraw $4 \mu \mathrm{L}$ of the mixture (with $200 \mathrm{ng}$ of siRNA) out into $1.5 \mu \mathrm{L}$ of $1 \%$ SDS and place on ice at time $0,5,10,15,20,30,45,60,75,90,105$ and $120 \mathrm{~min}$. For naked siRNA1, the same procedure was carried out only without incubation in the mixture with vesicles. Samples collected at different time points were analyzed using electrophoresis with $2 \%$ agarose gel.

\subsubsection{Cellular uptake study}

HeLa cells were cultured with Dulbecco's modified eagle medium (DMEM) with $10 \%$ fetal bovine serum (FBS), $1 \%$ penicillin and streptomycin (PS) under $5 \% \mathrm{CO}_{2}$ atmosphere at $37^{\circ} \mathrm{C}$.

In confocal studies, HeLa cells were seeded in a 6 -well tissue culture plate $(2 \mathrm{~mL}$ medium) with a density of $2.0 \times 10^{5}$ cells per well on the slides. After culturing $24 \mathrm{~h}$, 
the vesicle was added into the culture medium with the final concentration of $2 \mu \mathrm{M}$. After incubated with vesicles at different time, the culture medium was removed, following washing with the PBS for three times and then fixed with $4 \%$ formaldehyde at room temperature for $15 \mathrm{~min}$.

\subsubsection{In vitro cytotoxicity study}

MTT assay was used to investigate the cytotoxicity of supramolecular AIE vesicle, supramolecular AIE vesicle loaded with siPIK1, and supramolecular AIE vesicle loaded with siNC. The HeLa cells were seeded in 96-well plates $(200 \mu \mathrm{L}$ medium) and incubated for $24 \mathrm{~h}$. After the cell density reached $60 \%-70 \%$, the cells were incubated with the supramolecular AIE vesicle, supramolecular AIE vesicle loaded with siPIK1, and supramolecular AIE vesicle loaded with siNC, following with incubated $72 \mathrm{~h}$, respectively. After the medium removed, the fresh medium with $10 \%$ MTT was added, and incubated with for another $4 \mathrm{~h}$. The medium was removed carefully, following with adding $100 \mu \mathrm{L}$ DMSO. Finally, optical densities of the samples were measured using a microplate reader with the wavelength of $570 \mathrm{~nm}$ and $490 \mathrm{~nm}$.

\subsection{Results and discussion}

\subsubsection{Design and synthesis of the bola type supramolecular AIE vesicle}

The synthetic procedure of adamantane TPE was shown in Scheme 7. Compound 3 and TPE-2OH were synthesized by adapting procedures reported previously. Azide adamantane was obtained by amantadine reacting with the 2 -azidoacetic acid. For the synthesis of the compound 2 , compound 3 was reacted with bromoacetyl chloride in 
the presence of TEA in dichloromethane. Compound 2 was reacted with TPE- $2 \mathrm{OH}$ to give the compound 1 . At last, compound 1 was reacted with azide adamantane via click reaction to afford compound G1 as guest molecules. The overall chemical structures of $\mathrm{H} 1,4,3,2$, and G1 were confirmed by ${ }^{1} \mathrm{H} \mathrm{NMR},{ }^{13} \mathrm{C} \mathrm{NMR}$, and HRMS.
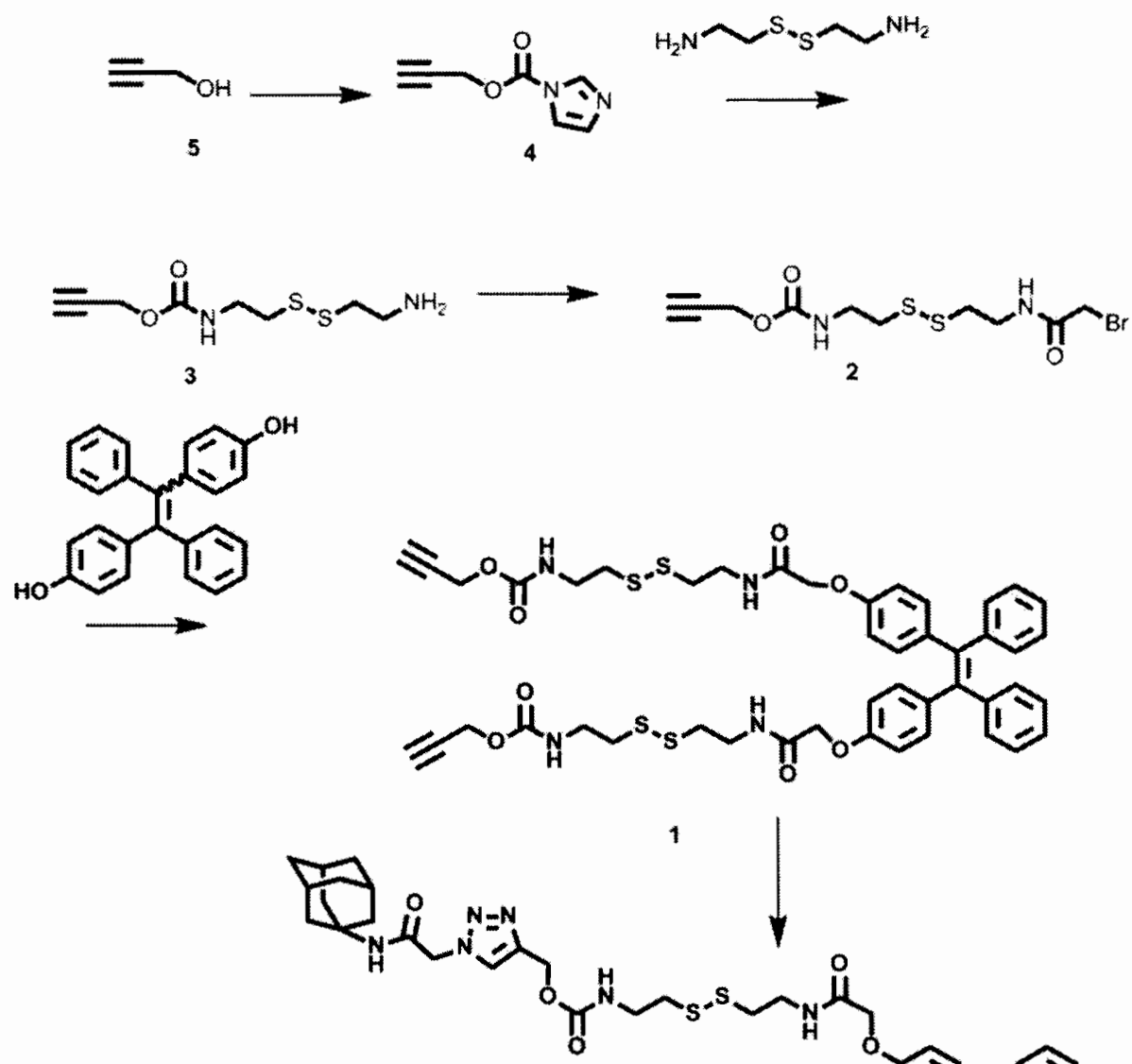

G1<smiles>[2H]c1ccc(Cc2ccccc2)cc1</smiles>

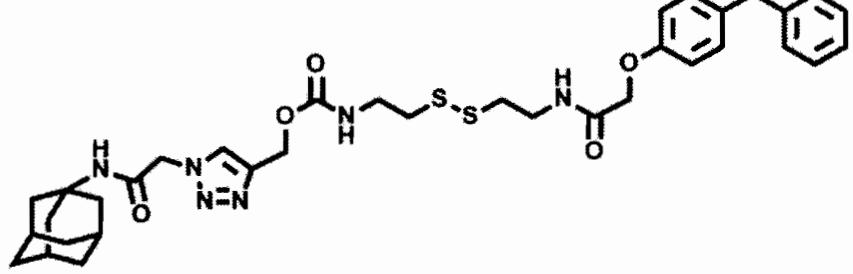

Scheme 7 Schematic illustration of the synthesis route and the structure of the guest molecule G1.

\subsubsection{Study of the AIE properties of the guest molecule G1.}

Due to containing of the TPE functional group in G1, the G1 exhibited typical AIE properties. As shown in Figure 52, a series of G1 solutions were prepared with the 
different $\mathrm{THF} / \mathrm{H}_{2} \mathrm{O}$ ratio, range from $0 \%$ to $95 \%$. G1 displayed no fluorescence emission after dissolved in THF. After increasing the fraction of the water, there also no fluorescence emission was observed, until the water fraction reached $60 \%$. Strong blue fluorescence emission was observed at the water fraction of $60 \%$, indicating that the G1 formed aggregate in this ratio. The fluorescence intensity at the $465 \mathrm{~nm}$ was also recorded, and a dramatic intensity increasing was observed at the $60 \%$ water fraction. Increasing of the fraction of the water result in the increased in the blue emission, suggesting that G1 had the strong AIE ability, which could be utilized to image the cancer cells.

a)

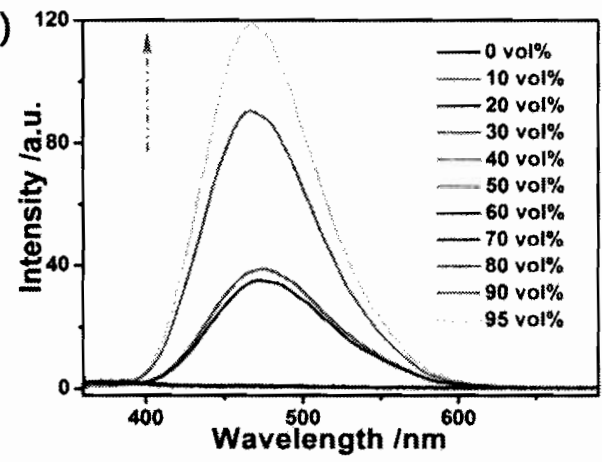

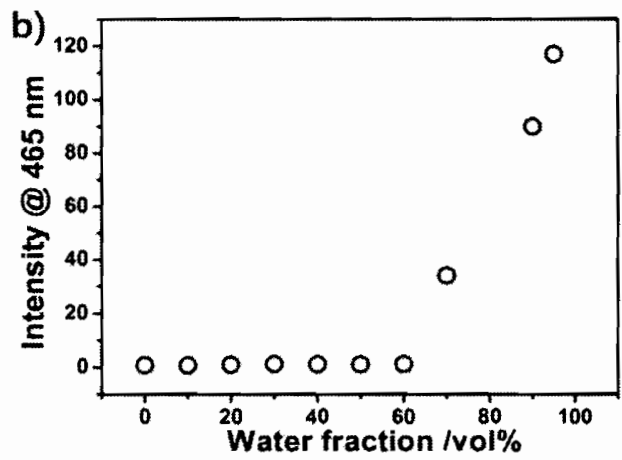

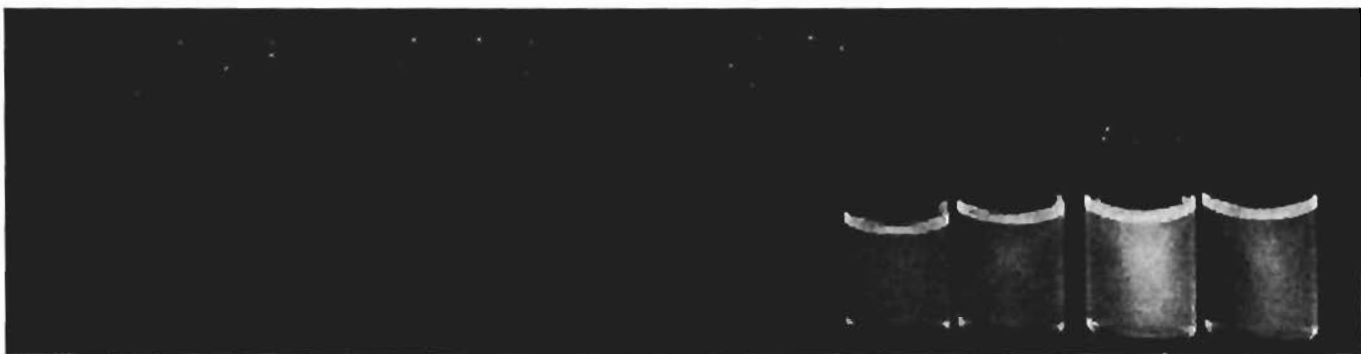

Figure 52 (a) The fluorescence spectrum of the $G 1$ with the different water fraction. (b) The intensity of the fluorescence at the $465 \mathrm{~nm}$ changes along the water fraction changes. (c) Photography of the G1 solution in different water fraction under the UV irradiation, the wavelength is $365 \mathrm{~nm}$. 
4.3.3 Self-assembly behaviors of the supramolecular amphiphiles bola type AIE vesicle

Because of supramolecular amphiphilic property, the host-guest inclusion complex can form higher order aggregates in water. To prepare amphiphilic supramolecular assembly, the Ada-TPE dissolved in DMSO was slowly injected into the dendritic cyclodextrin solution under the sonication with the ratio of $1: 2$, followed with dialysis against water. Dynamic light scattering (DLS) was employed to determine the size of the aggregate of the $\mathrm{H1}$ and $\mathrm{G} 1$ in aqueous solution. As shown in Figure 1(c), the vesicles exhibited an average hydrodynamic diameter about $94 \mathrm{~nm}$ with a narrow distribution. Transmission electron microscopy (TEM) images exhibited vesicle like morphology with the size about $100 \mathrm{~nm}$, which was in accordance with the results of the DLS, Figure 53 (a). The size of the vesicles was around $100 \mathrm{~nm}$, indicating its suitability for passive uptake by tumor tissue. The wall thickness of the vesicles was about $9 \mathrm{~nm}$, which was consistent with the simulation results, Figure 54 . The solution of the bola type TPE supramolecular amphiphilic vesicles with the concentration above the CAC (critical aggregation concentration) displayed obvious Tyndall effect, indicating that nanosize aggregates was formed by the bola type AIE supramolecular amphiphiles Figure 53 (d). The supramolecular vesicle was also displayed excellent stability in aqueous solution and could be stored for several months at room temperature. 
a)

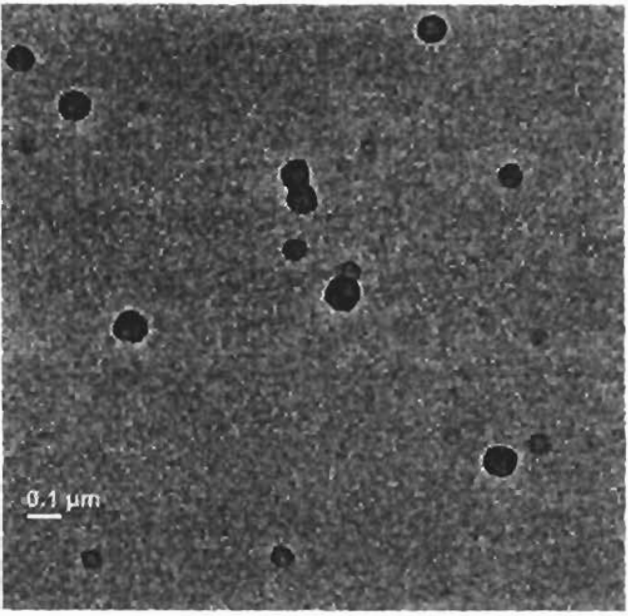

c)

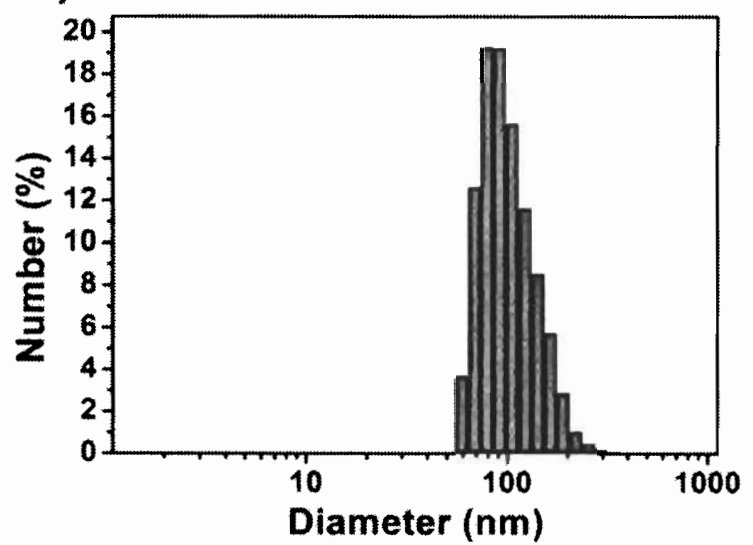

b)

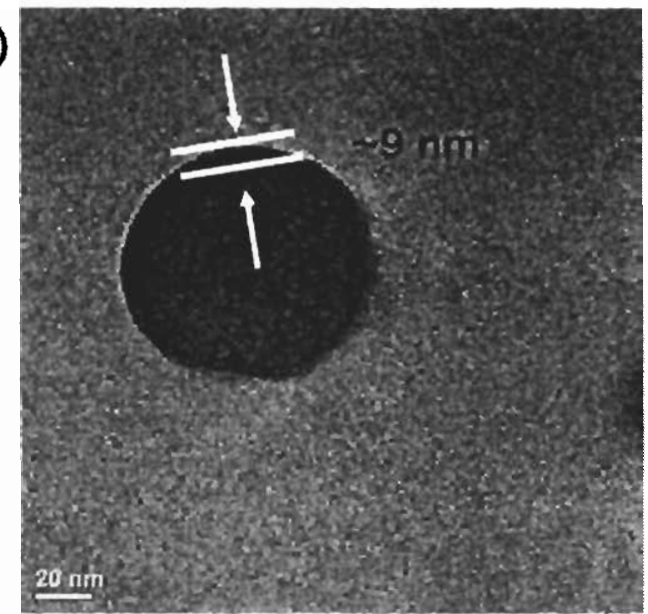

d)

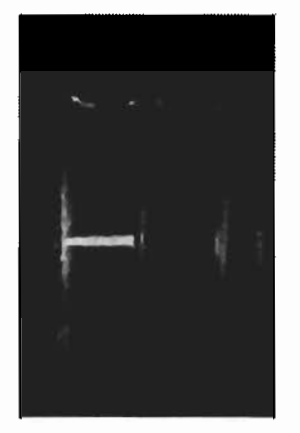

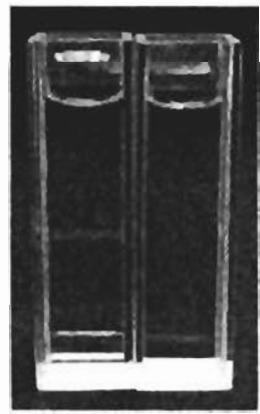

Figure 53 (a) TEM image of the vesicles self-assembled form the bola type TPE supramolecular amphiphilic vesicles between the $H 2$ and $G 1$, scale bar $=100 \mathrm{~mm}$. (b) Enlarge TEM image of the vesicles, the thickness is about $9 \mathrm{~nm}$, scale bar is $20 \mathrm{~nm}$. (c) DLS data of the vesicles self-assembled form the bola type TPE supramolecular amphiphilic vesicles. (d) Photography showing the Tyndall effect of the bola type TPE supramolecular amphiphilic vesicles, left: in the dark, right: in presence of light. 


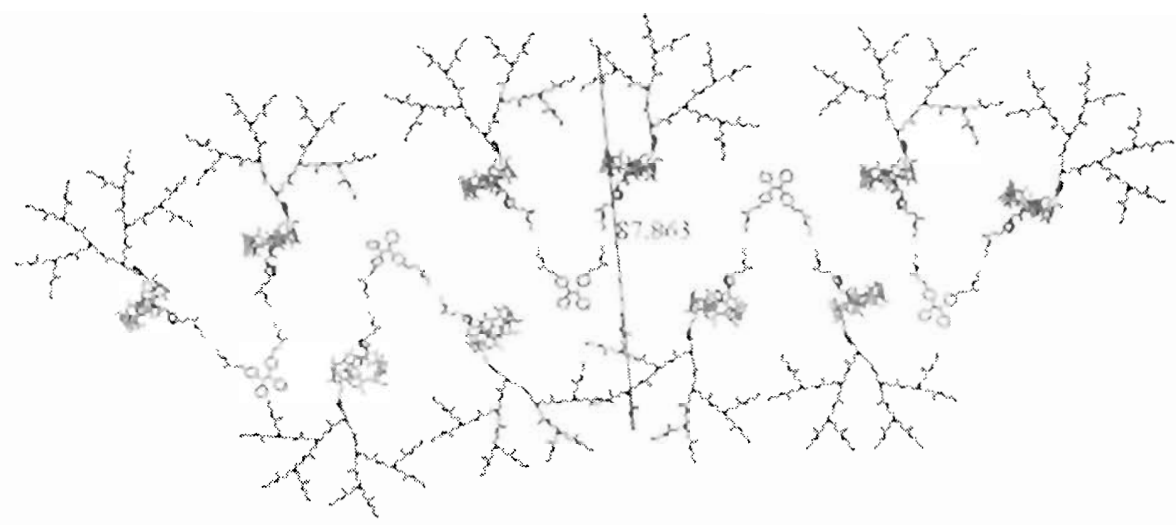

Figure 54 Simulation results of the stacking states of the bola type TPE supramolecular amphiphiles. The

thickness of the wall is about $8.7 \mathrm{~nm}$.

The CAC was determined by using the fluorescence method upon increasing the concentration of the bola TPE supramolecular amphiphilic vesicles range from 0.1 $\mu \mathrm{M}$ to $7 \mu \mathrm{M}$, which was developed by the Tang group. The fluorescence intensity at $480 \mathrm{~nm}$ excitation with $360 \mathrm{~nm}$ UV light, displayed linear increment upon the concentration of the supramolecular amphiphiles increasing. When the concentration reached $0.7 \mu \mathrm{M}$, the increment gradient became steeper, indicating the appearance of different aggregation state. The change in aggregation state suggested that the CAC determined was $0.7 \mu \mathrm{M}$. The proposed mechanism was shown below. Initially, the adamantane segment of the G1 was included in the cavity of the dendritic cyclodextrin formation of the supramolecular amphiphiles. The large dendritic cyclodextrin led that the rotation of the TPE was suppressed, followed with the appearance of the blue fluorescence emission. When the concentration of the supramolecular amphiphiles reached CAC, the supramolecular amphiphiles formed 
higher order supramolecular aggregate. TEM showed that the morphology was vesicle structure. The supramolecular amphiphiles was composed of the wall of the vesicle, and the rotation of the TPE segment was restricted stronger than in the free supramolecular amphiphiles. The compact stacking states of the TPE led to faster fluorescence increase, indicating that the supramolecular amphiphiles reached the CAC (Figure 55).
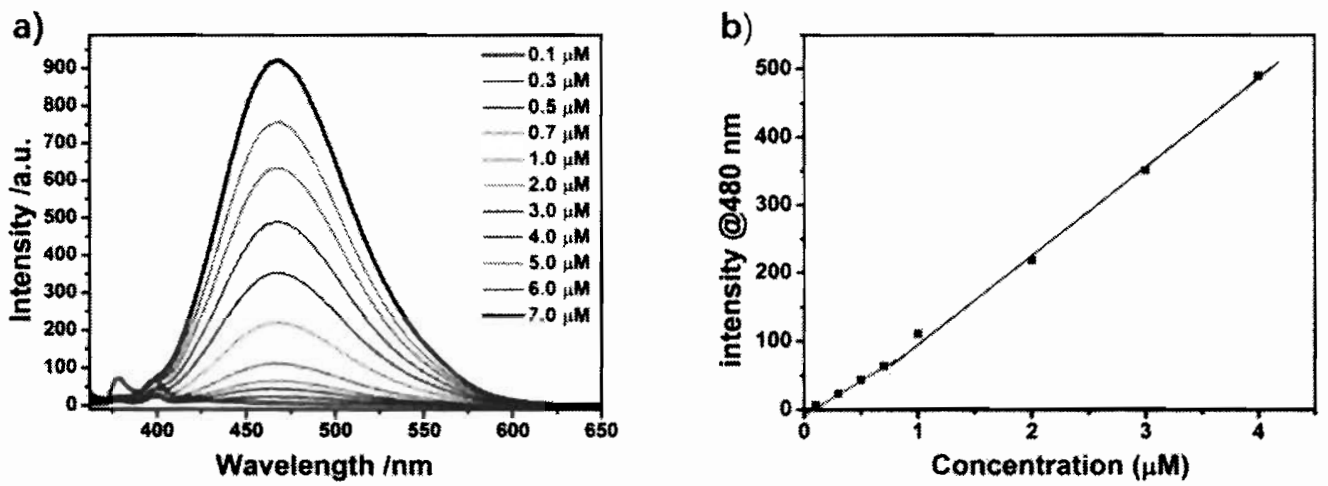

Figure 55 (a) Fluorescence spectrum of the bola type TPE supramolecular amphiphilic vesicle. Excitation wavelength is $360 \mathrm{~nm}$. (b) The intensity of the fluorescence at $480 \mathrm{~nm}$ of the different supramolecular amphiphiles.

\subsubsection{SiRNA delivery study}

\subsubsection{SiRNA binding study}

In our previous work, we also used dendritic prodrug supramolecular amphiphilic vesicle for combination cancer therapy. Because of the structure of the carriers significantly influencing the delivery efficacy of the siRNA, we hypothesized that the bola type AIE supramolecular vesicle maybe improve the siRNA binding efficiency. The N/P (terminal amines/phosphates) ratio was also optimized by using the electrophoretic mobility experiments in agarose gel. As shown in Figure 5, the siRNA 
was completely retarded at the N/P ratio at $20 / 1$, which is better than the previous work. The higher efficiency of the bola type AIE supramolecular vesicle delivery siRNA could be attributed to the special structure of the supramolecular amphiphiles. To test the siRNA stability after binding with the vesicles, the siRNA vesicles complexes were incubated with RNase A over $120 \mathrm{~min}$, and samples collected at different time points were analyzed using electrophoresis with $2 \%$ agarose gel, Figure 56.
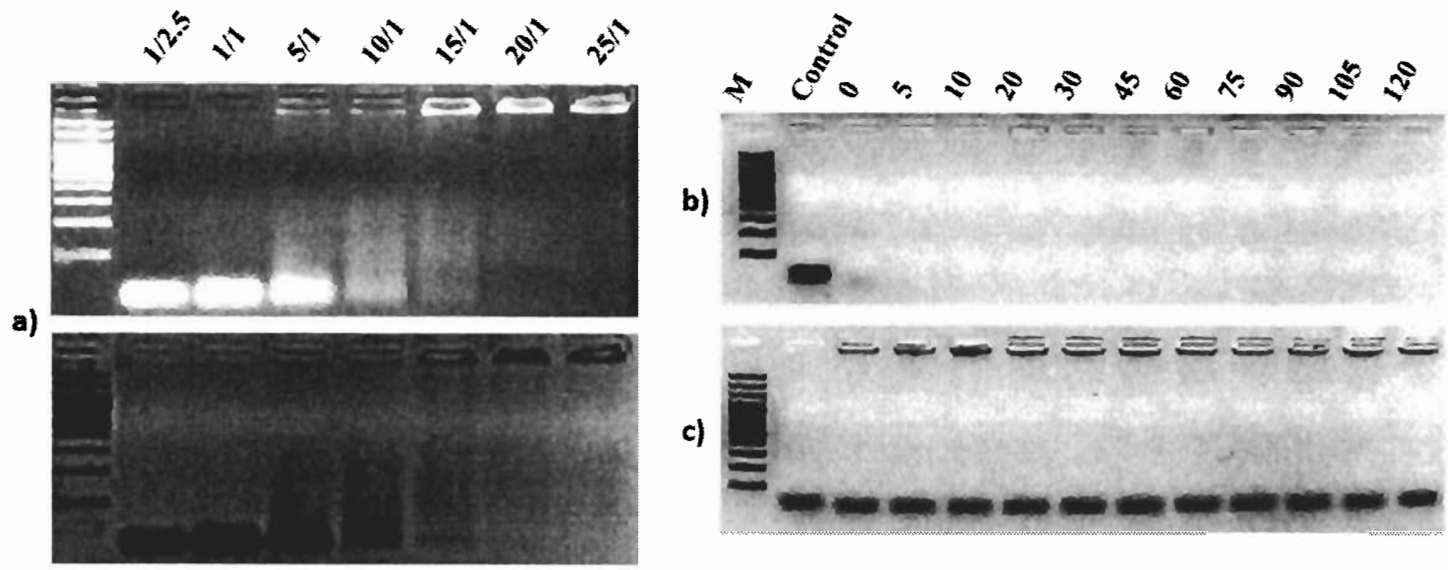

Figure 56 (a) Gel retardation of 200ng siRNA in agarose gel with vesicles at NiP charge ratios ranging from 1:2.5 to 25:1. (b) Gel retardation of the siplk1 under the treatment of the RNase degradation (c) The result of SIRNAivesicle complexes protect SIRNA from

TEM was employed to characterize the morphology of the bola type AIE supramolecular vesicle, and the results was shown below (Figure 57). From the TEM images, the aggregates exhibited vesicle like structures with the size about $50 \mathrm{~nm}$. The DLS data confirmed the results of the TEM, the vesicle exhibited narrow size distribution around $60 \mathrm{~nm}$. As mentioned in our previous work, this kind of size shrinkage could be attributed to the surface charge change inducing reassembly. The 
zeta potential was employed to characterize the surface charge of the supramolecular vesicles before and after loaded with siRNA. Absence of the siRNA, the supramolecular vesicle exhibited positive charge with the $+21 \mathrm{mV}$, while the supramolecular vesicle displayed $+17.2 \mathrm{mV}$ in presence of the siRNA, Figure 57 (d). The decrement of the positive charge indicated that the siRNA was successfully bonded with bola type TPE supramolecular vesicle.

a)

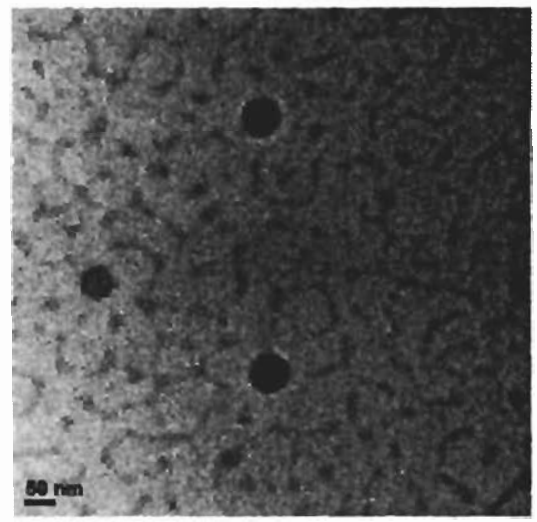

c)

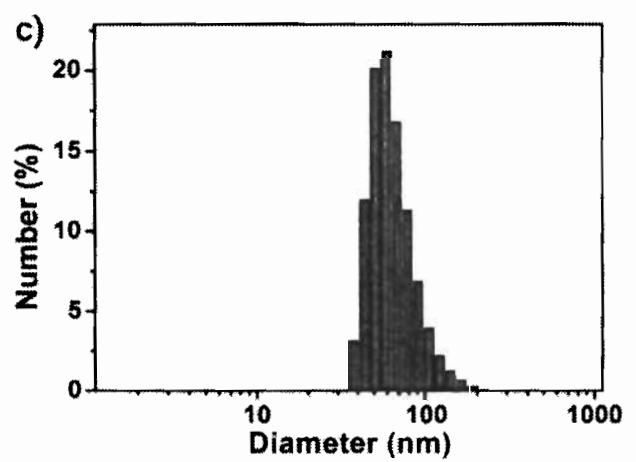

b)
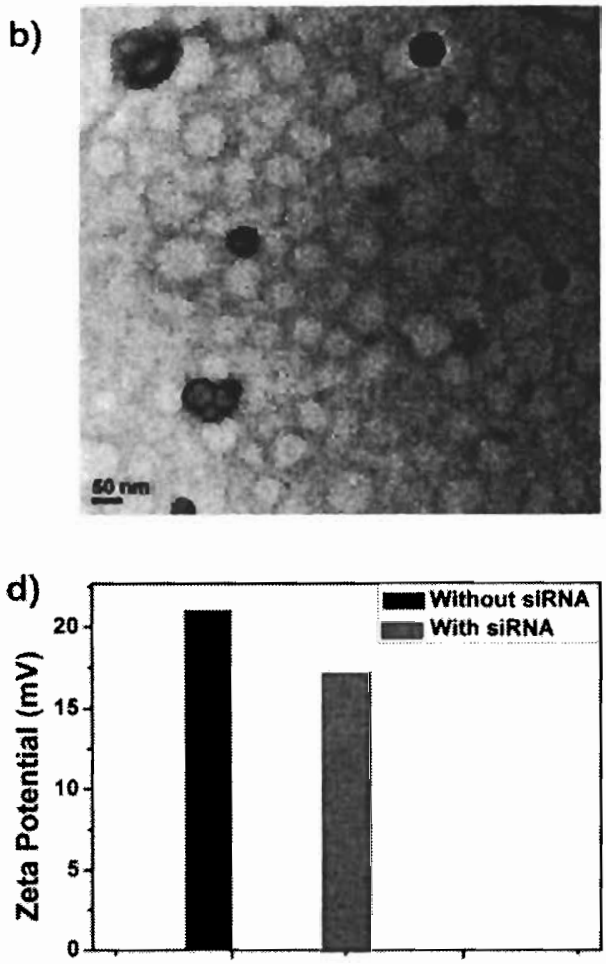

Figure 57 (a) and (b) TEM images of the bola type AIE vesicles after loaded with siRNA. The scale bar is $50 \mathrm{~nm}$. (c) DLS result of the bola type AIE vesicles after loaded with siRNA. (d) Zeta potential data of the bola type AIE vesicles absence of SIRNA and in presence of SIRNA.

\subsubsection{Cell uptake study}

To provide the evidence of the successfully siRNA delivery in cellular level, CLSM (confocal laser scanning microscope) was employed to characterized the cancer cells. 
As shown in Figure 58, after incubated with the siRNA loaded bola type TPE supramolecular vesicle, obvious blue fluorescence was observed under the excitation of the laser with DAPI channel. The blue fluorescence emission was generated from the TPE group, indicating that the vesicle was successfully uptake by the HeLa cells. After excitation of the cy3 channel, red fluorescence was observed, which was attributed to the emission of the cy 3 dye. The appearance of the cy 3 fluorescence suggested that the bola type TPE supramolecular vesicle could efficient deliver siRNA into the cancer cells.

a)
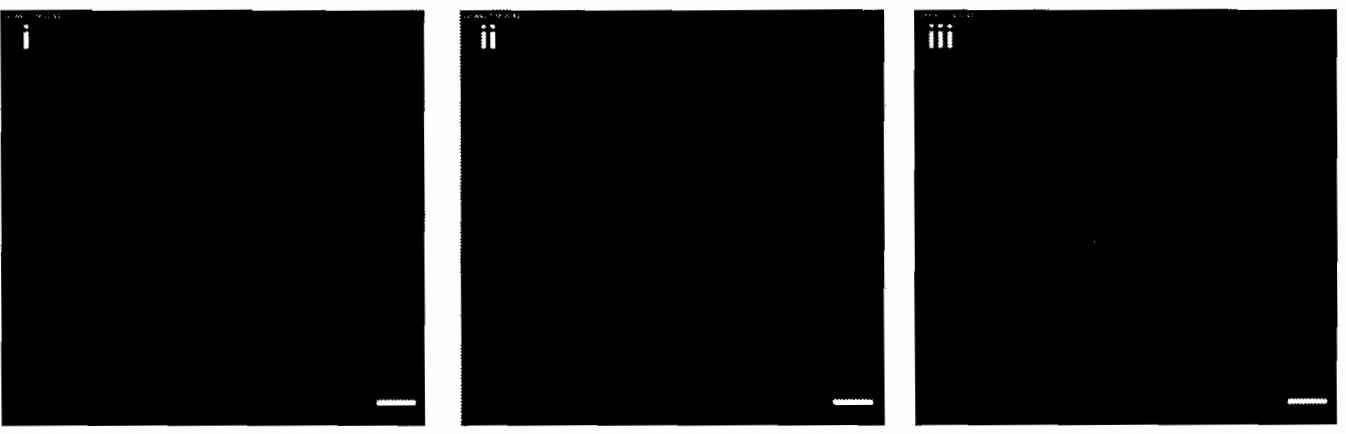

b) $\mathrm{i}$
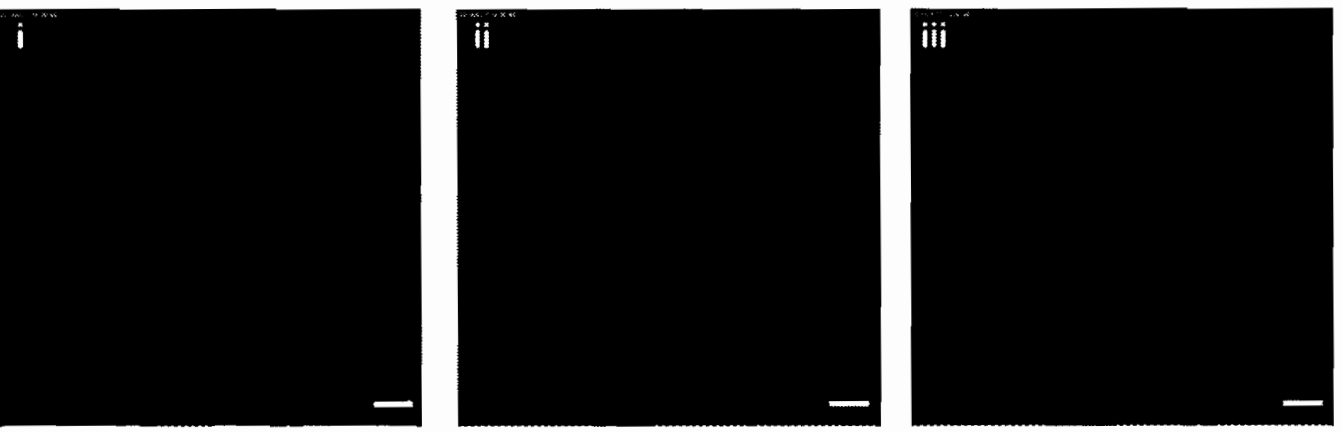

Figure 58 (a) Confocal fluorescence images of HeLa cells: HeLa cells incubated with the vesicles-cy3-siRNA complexes (2 $\mu \mathrm{M})$ for $12 \mathrm{~h}$; i) blue channel at $450 \pm 35 \mathrm{~mm}$, ii) cy3 channel. (iv) Overlap image generated from (i) and (ii) scale bar $=20 \mu \mathrm{m}$. (b) Enlarge confocal fluorescence images of HeLa cells. scale bar $=15 \mu \mathrm{m}$ 


\subsubsection{Cytotoxicity study}

MTT assay was utilized to characterize the cytotoxicity of the TPE supramolecular vesicle, negative control siRNA loaded TPE supramolecular vesicle, and siPIK1 loaded TPE supramolecular vesicle. The dendritic cyclodextrin had already been proved the safety as gene vector. The bola type TPE supramolecular vesicle was acted as control. As shown in Figure 59, compared with the control, negative control siRNA loaded TPE supramolecular vesicle exhibited slight improvement of cancer cells growth, while siPIK1 loaded TPE supramolecular vesicle displayed obviously growth suppression of the cancer cells. The results of the MTT assay suggested that the bola type TPE supramolecular vesicle successfully deliver the siPlK1 into the HeLa cells and efficiently inhibited the growth of the HeLa cells.

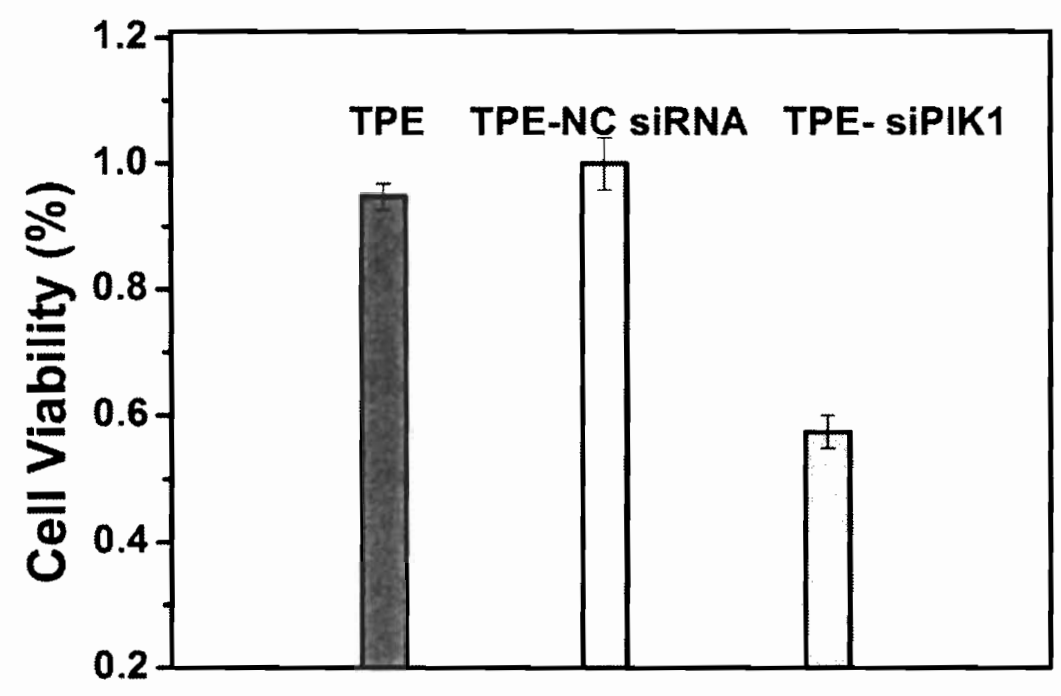

Figure 59 Cell viability of TPE CD (supramolecular amphiphilic vesicles). TPE-CD-siPIK1 complex, and TPE-CD-siNC Complex with the concentration of $10 \mu M$ and siRNA concentration of $100 \mathrm{nM}$. The HeLa cells were incubated for $72 \mathrm{~h} 37^{\circ} \mathrm{C}$. 


\subsection{Conclusion}

In summary, we developed a bola type TPE supramolecular vesicle as an efficient gene vector for delivery siRNA. In addition to acting as hydrophobic segment, the TPE segment was also employed as AIE imaging fluorophore. The special bola structure of the supramolecular amphiphile provided the stronger siRNA binding ability than the normal supramolecular vesicles. In cell study, the bola type TPE supramolecular vesicle exhibited efficient siRNA delivery ability. MTT assay displayed that siPIK1 loaded bola type TPE supramolecular vesicle evidently inhibited the growth of the cancer cells. Our work provided a new approach to design and fabricate siRNA carriers by using new types of supramolecular amphipiles. In future, more design and types of supramolecular amphiphiles such as gemini for siRNA delivery could be tested. 


\section{Conclusion and outlook}

This thesis illustrates my $\mathrm{PhD}$ research for the past four years, which mainly focus on prodrug construction, supramolecular amphiphilic prodrug nanosize aggregates fabrication, and development of supramolecular amphiphiles as safe and efficient siRNA delivery vectors.

To alleviate the side effects of the chemotherapy, construction of the prodrugs has been a popular strategy. Prodrugs show negligible toxicity in the normal tissues and can be activated to convert its active analog after triggered by the specific microenvironments of tumor tissues, or various external stimuli. Although prodrugs have multiple advantages as compared to free drugs, they also suffer from some disadvantages, including poor water solubility and poor tumor accumulation. Drug nanocarriers used to deliver prodrug also faced problems such as premature leakage, low drug loading content, minimal renal clearance, and poor drug release efficacy. Development of the amphiphilic prodrugs which can self-assemble to form nanoparticles has thus attracted attention to overcome such limitation.

In my first work, I synthesized an amphiphilic prodrug, which was composed of three components including anticancer drug chlorambucil as the therapeutic segment, naphthalimide as fluorescence responsive fluorophore, and mannose as targeting ligand. The mannose group acts as the hydrophilic component, while the fluorophore conjugated with the chlorambucil drug was employed as hydrophobic portion. The inherent amphiphilic property of the prodrug allowed the prodrug to self-assemble to form the nanaosize vesicle. The prodrug vesicle exhibited excellent cells 
discrimination via mannose receptor medicated endocytosis. After disulfide cleavage by the GSH, the prodrug was activated to release drug, accompanied with obvious fluorescence red-shift. This nano-platform showed excellent targeted imaging-guided drug delivery ability and has great potential application in cancer therapy.

After completion of the first project, we realized that synthesis and purification of amphiphilic prodrugs was challenging and time-consuming. To avoid complicated synthesis and purification procedures, host-guest chemistry inspired supramolecular amphiphilic aggregates were introduced. Adamantane modified CPT was synthesized as guest molecule, while the dendrimer-bearing cyclodextrin was prepared as host molecule. The separated synthesis of the drug and targeting component simplifies the synthesis procedures. The adamantane could fit into the cavity of the cyclodextrin to form supramolecular amphiphile, which self-assembled in aqueous solution to give supramolecular prodrug vesicle. Due to the positive charge on the surface of the vesicle, the supramolecular prodrug vesicle was also employed as siRNA carrier via electrostatic interaction. After internalization by the cancer cells, the disulfide bond was cleaved in presence of elevated amount of GSH, followed by the intramolecular cyclization to release free CPT, and the absorbance of the fluorophore also underwent obvious bathochromic shift. This change in fluorescence was employed for real time monitoring of the drug release as well as for cancer cell imaging. The supramolecular prodrug vesicle as siRNA carrier achieved dual therapy, exhibiting synergetic effect. This system provided a new strategy to deliver both prodrug and therapeutic gene, showing promising applications in cancer treatments. 
After promising results was obtained by harnessing supramolecular amphiphilic vesicle to delivery siRNA, we hypothesized that the structures of the supramolecular amphiphiles should affected the siRNA delivery ability. Thus we developed a bola type TPE supramolecular vesicle for siRNA delivery. The TPE was served as hydrophobic segment as well as imaging fluorophore. The dendrimer modified cyclodextrin was employed as hydrophilic segment as well as siRNA binding site. The supramolecular amphiphiles were self-assembled to afford supramolecular vesicle in aqueous solution. The bola type TPE prodrug efficiently delivered the siRNA into the cancer cells and exhibited improved siRNA binding affinity as compared to the normal supramolecular amphiphiles.

Upon completion of my current $\mathrm{PhD}$ research, I would like to continue my exploration of different supramolecular prodrug amphiphiles in order to achieve better therapeutic efficacy for cancer treatment. The incorporation of other different macrocycles such as calix[n]arene $(\mathrm{CA}[\mathrm{n}])$ and cucurbit[n] urils $(\mathrm{CB}[\mathrm{n}])$ to fabricate the supramolecular amphiphilic systems are possible modifications. Furthermore, another possible extension could be the variation of the guest molecule where other therapeutic agents such as photothermal and photodynamic agents could be used. The results obtained from current project tells the potential of supramolecular amphiphiles as a promising strategy for treatment of cancer and is worth further exploration. 


\section{References}

(1) Allen, T. M.; Cullis, P. R. Adv. Drug Deliv. Rev. 2013, 65 (1), 36-48.

(2) Lee, E. S.; Na, K.; Bae, Y. H. J. Control. Release 2005, 103 (2), 405-418.

(3) Zhao, J.; Zhang, H.; Sun, H.; Liu, Y. Chem. - An Eur. J. 2015, 21, 4457-4464.

(4) Luo, Z.; Hu, Y.; Cai, K.; Ding, X.; Zhang, Q.; Li, M.; Ma, X.; Zhao, Y. Biomaterials 2014, 35 (27), 7951-7962.

(5) Saha, K.; Agasti, S. S.; Kim, C.; Li, X.; Rotello, V. M. Chem. Rev. 2012, 112, 2739-2779.

(6) Lamprecht, A. Nat. Publ. Gr. 2015, 12 (4), 195-204.

(7) Shen, Y.; Jin, E.; Zhang, B.; Murphy, C. J.; Sui, M.; Zhao, J.; Wang, J.; Tang, 1.; Fan, M.; Kirk, E. Van; Murdoch, W. J. J. AM. CHEM. SOC. 2009, No. 5, 12780-12781.

(8) Peer, D.; Karp, J. M.; Hong, S.; Farokhzad, O. C.; Margalit, R.; Langer, R. Nat. Nanotechnol. 2007, 2 (12), 751-760.

(9) Fan, W.; Shen, B.; Bu, W.; Chen, F.; Zhao, K.; Zhang, S.; Zhou, L.; Peng, W.; Xiao, Q.; Xing, H.; Liu, J.; Ni, D.; He, Q.; Shi, J. J. Am. Chem. Soc. 2013, 135, 6494-6503.

(10) Celli, J. P.; Spring, B. Q.; Rizvi, I.; Evans, C. L.; Samkoe, K. S.; Verma, S.; Pogue, B. W.; Hasan, T. Chem. Rev. 2010, 110, 2795-2838.

(11) Chang, C.; Hsieh, M.; Lin, J.; Chang, T. Biomaterials 2012, 33 (3), 897-906.

(12) Rieger, J.; Freichels, H.; Imberty, A.; Putaux, J. L.; Delair, T.; Jerome, C.; Auzely-Velty, R. Biomacromolecules 2009, 10 (3), 651-657.

(13) Thambi, T.; Park, J. H.; Lee, D. S. Chem. Commun. 2016, 52, 8492-8500.

(14) Fang, J.; Nakamura, H.; Maeda, H. Adv. Drug Deliv. Rev. 2011, 63 (3), 136-151.

(15) Maeda, H.; Wu, J.; Sawa, T.; Matsumura, Y.; Hori, K. J. Control. Release 2000, 65 (1-2), 271284.

(16) Yoo, H. S.; Park, T. G. J. Control. Release 2004, 100 (2), 247-256.

(17) Huang, P.; Wang, D.; Su, Y.; Huang, W.; Zhou, Y.; Cui, D.; Zhu, X.; Yan, D. J. Am. Chem. Soc. 2014, 136 (33), 11748-11756.

(18) Wu, J.; Zhao, L.; Xu, X.; Bertrand, N.; Choi, W. I.; Yameen, B.; Shi, J.; Shah, V.; FaroKhzad, O. C. Angew. Chemie - Int. Ed. 2015, 54 (32), 9218-9223.

(19) Kelkar, S. S.; Reineke, T. M. Bioconjug. Chem. 2011, 22 (10), 1879-1903.

(20) Abeylath, S. C.; Ganta, S.; Iyer, A. K.; Amiji, M. Acc. Chem. Res. 2011, 44 (10), 1009-1017.

(21) Petros, R. a; DeSimone, J. M. Nat. Rev. Drug Discov. 2010, 9 (8), 615-627.

(22) Kuang, H.; Ku, S. H.; Kokkoli, E. Adv. Drug Deliv. Rev. 2016.

(23) Jin, H.; Kim, A.; Miyata, K.; Kataoka, K. Adv. Drug Deliv. Rev. 2016, 104, 61-77.

(24) Liang, J.; Liu, B. Bioeng. Transl. 2016, 1-13.

(25) Min, Y.; Mao, C.; Chen, S.; Ma, G.; Wang, J.; Liu, Y. Angew. Chem. Int. Ed. 2012, 6742-6747.

(26) Vallet-regí, M.; Balas, F.; Arcos, D. Angew. Chem. Int. Ed. 2007, 7548-7558.

(27) Liu, J.; Qi, Y.; Bhattacharjee, S.; Chilkoti, A. Angew. Chem. Int. Ed. 2015, 1002-1006.

(28) Chen, J.; Xu, X.; Chen, W.; Zhang, X. ACS Appl. Mater. Interfaces 2014, 593-598.

(29) Popat, A.; Ross, B. P.; Liu, J.; Jambhrunkar, S.; Kleitz, F.; Qiao, S. Z. Angew. Chem. Int. Ed. 2012, 12486-12489.

(30) Duncan, R. Nat. Rev. Cancer 2006, 6 (9), 688-701.

(31) Liu, G.; Wang, X.; Hu, J.; Zhang, G.; Liu, S. J. Am. Chem. Soc. 2014, 136 (20), 7492-7497.

(32) Gao, W.; Chan, J.; Farokhzad, O. C. Mol. Pharm. 2010, 7 (6), 1913-1920. 
(33) Mo, R.; Jiang, T.; DiSanto, R.; Tai, W.; Gu, Z. Nat Commun 2014, 5, 1-10.

(34) De, R.; Aili, D.; Stevens, M. M. Adv. Drug Deliv. Rev. 2012, 64 (11), 967-978.

(35) Sun, T.; Hao, A. Supramol. Chem. 2013, 37-41.

(36) Qiu, Y.; Park, K. Adv. Drug Deliv. Rev. 2012, 64, 49-60.

(37) Bhuniya, S.; Maiti, S.; Kim, E. J.; Lee, H.; Sessler, J. L.; Hong, K. S.; Kim, J. S. Angew. Chemie - Int. Ed. 2014, 53 (17), 4469-4474.

(38) Maiti, S.; Park, N.; Han, J. H.; Jeon, H. M.; Lee, J. H.; Bhuniya, S.; Kang, C.; Kim, J. S. J. Am. Chem. Soc. 2013, 135 (11), 4567-4572.

(39) Ahmed, M.; Narain, R. Nanomedicine (Lond). 2015, 10 (14), 2263-2288.

(40) Zhao, F.; Yin, H.; Li, J. Biomaterials 2014, 35 (3), 1050-1062.

(41) Stender, A. S.; Marchuk, K.; Liu, C.; Sander, S.; Meyer, M. W.; Smith, E. A.; Neupane, B.; Wang, G.; Li, J.; Cheng, J.; Huang, B.; Fang, N. Chem. Rev. 2013, 113, 2469-2527.

(42) Malakoutikhah, M.; Teixidó, M.; Giralt, E. Angew. Chem. Int. Ed. 2011, 7998-8014.

(43) Kim, E.; Kim, D.; Jung, H.; Lee, J.; Paul, S.; Selvapalam, N.; Yang, Y.; Lim, N.; Park, C. G.; Kim, K. Angew. Chem. Int. Ed. 2010, 2, 4405-4408.

(44) Lee, M. H.; Han, J. H.; Kwon, P. S.; Bhuniya, S.; Kim, J. Y.; Sessler, J. L.; Kang, C.; Kim, J. S. J. Am. Chem. Soc. 2012, 134 (2), 1316-1322.

(45) Lee, M. H.; Kim, J. S.; Sessler, J. L. Chem. Soc. Rev. 2015, 44 (13), 4185-4191.

(46) Lee, M. H.; Kim, J. Y.; Han, J. H.; Bhuniya, S.; Sessler, J. L.; Kang, C.; Kim, J. S. J. Am. Chem. Soc. 2012, 134 (30), 12668-12674.

(47) Cai, K.; He, X.; Song, Z.; Yin, Q.; Zhang, Y.; Uckun, F. M.; Jiang, C.; Cheng, J. J. Am. Chem. Soc. 2015, $137(10), 3458-3461$.

(48) Hu, X.; Hu, J.; Tian, J.; Ge, Z.; Zhang, G.; Luo, K.; Liu, S. J. Am. Chem. Soc. 2013, 135, 1761717629

(49) Liu, J.; Pang, Y.; Zhu, Z.; Wang, D.; Li, C.; Huang, W.; Zhu, X.; Yan, D. Biomacromolecules 2013, 14, 1627-1636.

(50) Liong, M.; Tamanoi, F.; Zink, J. I. ACS Nano 2008, 2, 889-896.

(51) Saravanakumar, G.; Kim, J.; Kim, W. J. Adv. Sci. 2016, 124.

(52) Yoon, H.-J.; Jang, W.-D. J. Mater. Chem. 2010, 211-222.

(53) Kumar, R.; Han, J.; Lim, H.; Ren, W. X.; Lim, J.; Kim, J.; Kim, J. S. J. Am. Chem. Soc. 2014, 1783617843.

(54) Zhang, Y.; He, Z.; Li, G. Talanta 2010, 81 (1-2), 591-596.

(55) Wang, Q.; Li, G.; Xiao, W.; Qi, H.; Li, G. Sensors Actuators B 2006, 119, 695-700.

(56) Ma, X.; Zhao, Y. Chem. Rev. 2014, 115 (15), 7794-7839.

(57) Hong, Y.; Lam, W. Y.; Zhong, B. Chem. Commun. 2009, 4332-4353.

(58) Liu, Y.; Deng, C.; Tang, L.; Qin, A.; Hu, R.; Sun, J. Z.; Tang, B. Z. J. Am. Chem. Soc. 2011, 133, 660-663.

(59) Diehn, M.; Cho, R. W.; Lobo, N. A.; Kalisky, T.; Dorie, M. J.; Kulp, A. N.; Qian, D.; Lam, J. S.; Ailles, L. E.; Wong, M.; Joshua, B.; Kaplan, M. J.; Wapnir, I.; Dirbas, F. M.; Somlo, G.; Garberoglio, C.; Paz, B.; Shen, J.; Lau, S. K.; Quake, S. R.; Brown, J. M.; Weissman, I. L.; Clarke, M. F. Nature 2009, 458, 6-11.

(60) Lippert, A. R.; Bittner, G. C. V. A. N. D. E.; Chang, C. J. Acc. Chem. Res. 2011, 44, 793-804.

(61) Nicco, C.; Ch, C.; Laurent, A. Int. J. Cancer 2006, 48, 41-48.

(62) Trachootham, D.; Alexandre, J.; Huang, P. Nat. Publ. Gr. 2009, 8 (7), 579-591. 
(63) Fruehauf, J. P.; Meyskens, F. L. Clin Cancer Res 2007, 13, 789-794.

(64) Noh, J.; Kwon, B.; Han, E.; Park, M.; Yang, W.; Cho, W.; Yoo, W.; Khang, G.; Lee, D. Nat. Commun. 2015, 6, 1-9.

(65) Dai, J.; Lin, S.; Cheng, D.; Zou, S.; Shuai, X. Angew. Chemie - Int. Ed. 2011, 50 (40), 9404-9408.

(66) Ma, X.; Zhao, Y.; Liang, X. J. Acc. Chem. Res. 2011, 44 (10), 1114-1122.

(67) Zhao, Y.; Luo, Z.; Li, M.; Qu, Q.; Ma, X.; Yu, S. H.; Zhao, Y. Angew. Chemie - Int. Ed. 2015, 54 (3), 919-922.

(68) Yan, H.; Teh, C.; Sreejith, S.; Zhu, L.; Kwok, A.; Fang, W.; Ma, X.; Nguyen, K. T.; Korzh, V.; Zhao, Y. Angew. Chemie - Int. Ed. 2012, 51 (33), 8373-8377.

(69) Fluorogen, E. T. R.; Chen, S.; Hong, Y.; Liu, Y.; Liu, J.; Leung, C. W. T.; Li, M.; Kwok, R. T. K.; Zhao, E.; Lam, J. W. Y.; Yu, Y.; Tang, B. Z. 2013.

(70) Wang, X.; Stolwijk, J. A.; Lang, T.; Sperber, M.; Meier, R. J.; Wegener, J.; Wolfbeis, O. S. J. Am. Chem. Soc. 2012, 134, 17011-17014.

(71) Viricel, W.; Mbarek, A.; Leblond, J. Angew. Chem. Int. Ed. 2015, 12743-12747.

(72) Moitra, P.; Kumar, K.; Kondaiah, P.; Bhattacharya, S. Angew. Chem. Int. Ed. 2014, 1113-1117.

(73) Ghosh, A.; Haverick, M.; Stump, K.; Yang, X.; Tweedle, M. F.; Goldberger, J. E. 2012, 10-13.

(74) Li, D.; Song, J.; Yin, P.; Simotwo, S.; Bassler, A. J.; Aung, Y.; Roberts, J. E.; Hardcastle, K. I.; Hill, C. L.; Liu, T. J. Am. Chem. Soc 2011, 14010-14016.

(75) Dissolution, I.; Zhu, G. J. Am. Chem. Soc. 2011, 133, 8778-8781.

(76) Su, J.; Chen, F.; Cryns, V. L.; Messersmith, P. B. J. Am. Chem. Soc. 2011, 133, 11850-11853.

(77) Ghosh, A.; Haverick, M.; Stump, K.; Yang, X.; Tweedle, M. F.; Goldberger, J. E. J. Am. Chem. Soc 2012, 134, 3647-3650.

(78) Mackay, J. A.; Chen, M.; Mcdaniel, J. R.; Liu, W.; Simnick, A. J. Nat. Mater. 2009, 8 (12), 993999.

(79) Li, S.; Liu, L.; Jia, H.; Qiu, W.; Rong, L.; Cheng, H.; Zhang, X. Chem. Commun. 2014, 50, 1185211855.

(80) Brieke, C.; Rohrbach, F.; Gottschalk, A.; Mayer, G.; Heckel, A. Angew. Chem. Int. Ed. 2012, 8446-8476.

(81) Lin, Q.; Huang, Q.; Li, C.; Bao, C.; Liu, Z.; Li, F. J. AM. CHEM. SOC. 2010, 132, 10645-10647.

(82) Haas, K. L.; Franz, K. J. Chem. Rev. 2009, 109, 4921-4960.

(83) Fan, N.; Cheng, F.; Ho, J. A.; Yeh, C. Angew. Chem. Int. Ed. 2012, 8806-8810.

(84) Jain, P. K.; Karunakaran, D.; Friedman, S. H. Angew. Chem. Int. Ed. 2013, 1404-1409.

(85) Karakoti, A. S.; Das, S.; Thevuthasan, S.; Seal, S. Angew. Chem. Int. Ed. 2011, 1980-1994.

(86) Zhang, Y.; Yin, Q.; Yin, L.; Ma, L.; Tang, L.; Cheng, J. Angew. Chem. Int. Ed. 2013, 6435-6439.

(87) Hu, X.; Tian, J.; Liu, T.; Zhang, G.; Liu, S. Macromolecules 2013, 6243-6256.

(88) Fomina, N.; Sankaranarayanan, J.; Almutairi, A. Adv. Drug Deliv. Rev. 2012, 64 (11), 10051020.

(89) Sherwood, R. F. Adv. Drug Deliv. Rev. 1996, 269-288

(90) Kumar, R.; Kim, E.; Han, J.; Lee, H.; Sup, W.; Min, H.; Bhuniya, S.; Seung, J.; Soo, K. Biomaterials 2016, 104, 119-128.

(91) Liu, P.; Xu, J.; Yan, D.; Zhang, P.; Zeng, F.; Li, B. Chem. Commun 2015, 9567-9570.

(92) Li, M.; Teh, C.; Ang, C. Y.; Tan, S. Y.; Luo, Z.; Qu, Q.; Zhang, Y.; Korzh, V.; Zhao, Y. Adv. Funct. Mater. 2015, 25 (35), 5602-5610.

(93) Liu, J.; Bu, W.; Pan, L.; Shi, J. Angew. Chem. Int. Ed. 2013, 4375-4379. 
(94) Xie, J.; Lee, S.; Chen, X. Adv. Drug Deliv. Rev. 2010, 62 (11), 1064-1079.

(95) Jana, A.; Nguyen, K. T.; Li, X.; Zhu, P.; Tan, N. S.; Agren, H.; Zhao, Y. ACS Nano 2014, 8 (6), 5939-5952.

(96) Wang, J.; Mi, P.; Lin, G.; Xiáng, Y.; Wáng, J.; Liu, G.; Chen, X. Adv. Drug Deliv. Rev. 2016, 44-60.

(97) Thapa, P.; Li, M.; Bio, M.; Rajaputra, P.; Nkepang, G.; Sun, Y.; Woo, S.; You, Y. J. Med. Chem 2016, 3204-3214.

(98) Zhao, Z.; Chen, B.; Geng, J.; Chang, Z.; Aparicio-ixta, L.; Nie, H.; Goh, C. C.; Ng, L. G.; Qin, A.; Ramos-ortiz, G.; Liu, B. Part. Part. Syst. Charact. 2014, 481-491.

(99) Ding, D.; Goh, C. C.; Feng, G.; Zhao, Z.; Liu, J.; Liu, R.; Tomczak, N.; Geng, J.; Tang, B. Z.; Ng, L. G.; Liu, B. Adv. Mater 2013, 6083-6088.

(100) Geng, J.; Li, K.; Ding, D.; Zhang, X.; Qin, W.; Liu, J. small 2012, 3655-3663.

(101) Parthasarathy, V.; Fery-forgues, S.; Campioli, E.; Recher, G.; Terenziani, F.; Blanchard-desce, M. samll 2011, 3219-3229.

(102) Gary-bobo, M.; Mir, Y.; Rouxel, C.; Brevet, D.; Basile, I.; Maynadier, M.; Vaillant, O.; Mongin, O.; Blanchard-desce, M.; Mor, A.; Garcia, M.; Durand, J.; Raehm, L. Angew. Chem. Int. Ed. 2011, 11425-11429.

(103) Babin, J.; Pelletier, M.; Lepage, M.; Allard, J.; Morris, D. Angew. Chem. Int. Ed. 2009, 33293332.

(104) Zhao, Z.; Chen, B.; Geng, J.; Chang, Z.; Aparicio-ixta, L.; Nie, H.; Goh, C. C.; Ng, L. G.; Qin, A.; Ramos-ortiz, G.; Liu, B. 2014, 481-491.

(105) Yang, P.; Yang, Y.; Gao, Y.; Wang, Y.; Zhang, J.; Lin, Y.; Dai, L.; Li, J.; Wang, L.; Wang, H. Adv. Opt. Mater. 2015, 646-651.

(106) Rosenbaum, I.; Harnoy, A. J.; Tirosh, E.; Buzhor, M.; Segal, M.; Frid, L. J. Am. Chem. Soc. 2015, 137, 2276-2284.

(107) Wang, X.; Hu, J.; Liu, G.; Tian, J.; Wang, H.; Gong, M.; Liu, S. J. Am. Chem. Soc. 2015, 137, 15262-15275.

(108) Yu, C.; Wu, Y.; Zeng, F.; Li, X.; Shi, J.; Wu, S. Biomacromolecules 2013, 4507-4514.

(109) Iyer, A. K.; Singh, A.; Ganta, S.; Amiji, M. M. Adv. Drug Deliv. Rev. 2013, 65 (13-14), 17841802.

(110) Francesco M Veronese; Schiavon, O.; Pasut, G.; Mendichi, R.; Andersson, L.; Tsirk, A.; Ford, J.; Wu, G.; Duncan, R. Bioconjug. Chem. 2005, 16 (4), 775-784.

(111) Hilgenbrink, A. R.; Low, P. S. J. Pharm. Sci. 2005, 94 (10), 2135-2146.

(112) Rautio, J.; Kumpulainen, H.; Heimbach, T.; Oliyai, R.; Oh, D.; Jarvinen, T.; Savolainen, J. Nat. Rev. Drug Discov. 2008, 7 (3), 255-270.

(113) Liu, P.; Xu, J.; Yan, D.; Zhang, P.; Zeng, F.; Li, B.; Wu, S. Chem. Commun. 2015, 51, 9567-9570.

(114) Glunde, K.; Artemov, D.; Penet, M.; Jacobs, M. A.; Bhujwalla, Z. M. Chem. Rev 2010, 110, 3043-3059.

(115) Yu, G.; Zhou, X.; Zhang, Z.; Han, C.; Mao, Z.; Gao, C.; Huang, F. 2012.

(116) Pires, M. M.; Chmielewski, J.; Lafayette, W. Org.Lett. 2008, 10, 3809-3812.

(117) Kawakami, S.; Sato, A.; Nishikawa, M.; Yamashita, F.; Hashida, M. Gene Ther. 2000, 7 (4), 292299.

(118) Jin, S.; Ju, M.; Chan, I.; Roberts, T. M. Adv. Drug Deliv. Rev. 2016, 104, 2-15.

(119) Legigan, T.; Clarhaut, J.; Tranoy-opalinski, I.; Monvoisin, A.; Renoux, B.; Thomas, M.; Pape, A. Le; Lerondel, S.; Papot, S. Angew. Chem. Int. Ed 2012, 11606-11610. 
(120) Liang, D.; Miller, G. H.; Tranmer, G. K. Curr. Med. Chem. 2015, 4313-4325.

(121) Connor, L. J. O.; Cazares-körner, C.; Saha, J.; Evans, C. N. G.; Stratford, M. R. L.; Hammond, E. M.; Conway, S. J. Nat. Protoc. 2016, 11, 781-794.

(122) Karnthaler-benbakka, C.; Groza, D.; Kryeziu, K.; Pichler, V.; Roller, A.; Berger, W.; Heffeter, P.; Kowol, C. R. Angew. Chem. Int. Ed. 2014, 12930-12935.

(123) Park, T.; Zimmerman, S. C.; Nakashima, S. J. AM. CHEM. SOC. 2005, 8, 6520-6521.

(124) Devadasu, V. R.; Bhardwaj, V.; Kumar, M. N. V. R. Chem. Rev. 2013, 113, 1686-1735.

(125) De Cock, L. J.; De Koker, S.; De Geest, B. G.; Grooten, J.; Vervaet, C.; Remon, J. P.; Sukhorukov, G. B.; Antipina, M. N. Angew. Chemie - Int. Ed. 2010, 49 (39), 6954-6973.

(126) Hoare, T. R.; Kohane, D. S. Polym. with aligned carbon Nanotub. Act. Compos. Mater. 2008, 49 (8), 1993-2007.

(127) Ramirez, M.; Guan, D.; Ugaz, V.; Chen, Z. J. Am. Chem. Soc. 2013, 135, 5290-5293.

(128) Singh, S.; Topuz, F.; Hahn, K.; Albrecht, K.; Groll, J. Angew. Chem. Int. Ed. 2013, 3000-3003.

(129) Mura, S.; Nicolas, J.; Couvreur, P. Nat. Publ. Gr. 2013, 12 (11), 991-1003.

(130) Lee, M. H.; Yang, Z.; Lim, C. W.; Lee, Y. H.; Dongbang, S.; Kang, C.; Kim, J. S. Chem. Rev. 2013, 113, 5071-5109.

(131) Online, V. A.; Zhao, J.; Liu, X.; Wang, H.; Jin, Q.; Ji, J. Polym. Chem. 2014, 1843-1847.

(132) Grove, T. Z.; Osuji, C. O.; Forster, J. D.; Dufresne, E. R.; Regan, L. J. AM. CHEM. SOC. 2010, 132, 14024-14026.

(133) Kabanov, A. V; Vinogradov, S. V. Angew. Chem. Int. Ed 2009, 5418-5429.

(134) Soussan, E.; Cassel, S.; Blanzat, M.; Rico-lattes, I. Angew. Chem. Int. Ed. 2009, 274-288.

(135) Vulic, K.; Shoichet, M. S. J. Am. Chem.Soc 2012, 134, 882-885.

(136) Li, J.; Jun, X. Adv. Drug Deliv. Rev. 2008, 60, 1000-1017.

(137) Li, X.; Li, J.; Gao, Y.; Kuang, Y.; Shi, J.; Xu, B. J. AM. CHEM. SOC. 2010, 132, 17707-17709.

(138) Gao, Y.; Kuang, Y.; Guo, Z.; Guo, Z.; Krauss, I. J.; Xu, B. J. AM. CHEM. SOC. 2009, 131, 13576 13577.

(139) Liu, J.; Jiang, X.; Ashley, C.; Brinker, C. J. J. AM. CHEM. SOC. 2009 2009, 131, 7567-7569.

(140) Volodkin, D. V; Skirtach, A. G.; Möhwald, H. Angew. Chem. Int. Ed 2009, 1807-1809.

(141) Volodkin, D. V; Skirtach, A. G.; Möhwald, H. 2009, 1807-1809.

(142) Wang, Y.; Zhang, Y.; Wang, Y.; Liu, Y. 2015.

(143) Lee, J. S.; Feijen, J. J. Control. Release 2012, 161 (2), 473-483.

(144) Marguet, M.; Edembe, L.; Lecommandoux, S. Angew. Chem. Int. Ed. 2012, 1173-1176.

(145) Li, F.; Wolf, F. A. De; Marcelis, A. T. M.; Sudhölter, E. J. R.; Stuart, M. A. C.; Leermakers, F. A. M. Angew. Chem. Int. Ed. 2010, 9947-9950.

(146) Georgieva, J. V; Brinkhuis, R. P.; Stojanov, K.; Weijers, C. A. G. M.; Zuilhof, H.; Rutjes, F. P. J. T.; Hoekstra, D.; Hest, J. C. M. Van; Zuhorn, I. S. Angew. Chem. Int. Ed. 2012, 8339-8342.

(147) Wang, L.; Chierico, L.; Little, D.; Patikarnmonthon, N.; Yang, Z.; Azzouz, M.; Madsen, J.; Armes, S. P.; Battaglia, G. Angew. Chem. Int. Ed. 2012, 11122-11125.

(148) Yu, G.; Jie, K.; Huang, F. Chem. Rev. 2015, 115, 7240-7303.

(149) Online, V. A.; Wu, J.; Sun, S.; Feng, X.; Shi, J.; Hu, X.; Wang, L. Chem. Commun. 2014, 91229125.

(150) Jiao, D.; Geng, J.; Loh, X. J.; Das, D.; Lee, T.; Scherman, O. A. Angew. Chem. Int. Ed. 2012, 9633-9637. 
(151) Nguyen, T.; David, T.; Wang, D.; Belinda, N.; Klaus, M. J. Am. Chem. Soc. 2011, 133, 1119411204.

(152) Zhang, J.; Ma, P. X. Angew. Chem. Int. Ed. 2009, 964-968.

(153) Zhang, J.; Ma, P. X. Adv. Drug Deliv. Rev. 2013, 65 (9), 1215-1233.

(154) Online, V. A.; Song, N.; Chen, D.; Qiu, Y.; Yang, X.; Xu, B.; Tian, W.; Yang, Y. Chem. Commun. 2014, 8231-8234.

(155) Cao, Y.; Li, Y.; Hu, X.; Zou, X.; Xiong, S.; Lin, C.; Wang, L. Chem. Mater 2015, 27, 1110-1119.

(156) Mellet, C. O. Chem. Soc. Rev. 2011, 1586-1608.

(157) Davis, M. E.; Brewster, M. E. Nat. Rev. Drug Discov. 2004, 1023-1035.

(158) Srinivasachari, S.; Fichter, K. M.; Reineke, T. M. J. AM. CHEM. SOC. 2008, 130, 4618-4627.

(159) Sun, T.; Guo, Q.; Zhang, C.; Hao, J.; Xing, P.; Su, J.; Li, S.; Hao, A.; Liu, G. Langmuir 2012, 28, 8625-8636.

(160) Jing, J.; Auzély-Velty, R. Chem. Mater 2013, 25, 3867-3873.

(161) Lee, J. A. E. W.; Samal, S.; Selvapalam, N.; Kim, H.; Kim, K. Acc. Chem. Res. 2003, 36, 621-630.

(162) Liu, S.; Shukla, A. D.; Gadde, S.; Wagner, B. D.; Kaifer, A. E.; Isaacs, L. Angew. Chem. Int. Ed. 2008, 2657-2660.

(163) Lucas, D.; Minarni, T.; lannuzzi, G.; Cao, L.; Wittenberg, J. B.; Anzenbacher, P.; Isaacs, L. J. Am. Chem. Soc. 2011, 133, 17966-17976.

(164) Ma, D.; Zhang, B.; Hoffmann, U.; Sundrup, M. G.; Eikermann, M.; Isaacs, L. Angew. Chem. Int. Ed 2012, 11358-11362.

(165) Gallego-yerga, L.; Gonzulez-, M. J.; Mayordomo, N.; Santoyo-, F.; Benito, J. M.; Mellet, O.; Mendicuti, F.; García, J. M. Chem. Eur. J. 2014, 6622-6627.

(166) Ma, D.; Hettiarachchi, G.; Nguyen, D.; Zhang, B.; Wittenberg, J. B.; Zavalij, P. Y.; Briken, V.; Isaacs, L. Nat.Chem 2012, 503-510.

(167) Chen, Y.; Chen, C.; Hung, Y.; Chou, C.; Liu, T.; Liang, M.; Chen, C.; Mou, C. J. Am. Chem. Soc. 2013, 2013, 135, 1516-1523.

(168) Zhu, C.; Lu, C.; Song, X.; Yang, H.; Wang, X. J. Am. Chem. Soc 2011, 133, 1278-1281.

(169) Singh, N.; Karambelkar, A.; Gu, L.; Lin, K.; Miller, J. S.; Chen, C. S.; Sailor, M. J.; Bhatia, S. N. J. Am. Chem. Soc. 2011, 133, 19582-19585.

(170) Pan, L.; He, Q.; Liu, J.; Chen, Y.; Zhang, L.; Shi, J. J. Am. Chem. Soc 2012, 134, 5722-5725.

(171) Zhang, J.; Yuan, Z.; Wang, Y.; Chen, W.; Luo, G.; Cheng, S.; Zhuo, R.; Zhang, X. J. Am. Chem. Soc. 2013, 135, 5068-5073.

(172) Luo, Z.; Cai, K.; Hu, Y.; Zhao, L.; Liu, P.; Duan, L.; Yang, W. Angew. Chem. Int. Ed. 2011, 640643.

(173) Arima, H.; Motoyama, K.; Higashi, T. Adv. Drug Deliv. Rev. 2013, 65 (9), 1204-1214.

(174) Yu, S.; Zhang, Y.; Wang, X.; Zhen, X.; Zhang, Z.; Wu, W.; Jiang, X. Angew. Chem. Int. Ed. 2013, 7272-7277.

(175) He, Q.; Ao, Y.; Huang, Z.; Wang, D. Angew. Chem. Int. Ed. 2015, $11785-11790$.

(176) Nakase, I.; Gräslund, A.; Futaki, S. Acc. Chem. Res 2012, 45, 1132-1139.

(177) Song, S.; Zheng, H.; Li, D.; Wang, J.; Feng, H.; Zhu, Z.; Chen, Y.; Zheng, Y. Org. Lett. 2014, 16, 2170-2173.

(178) Yu, G.; Tang, G.; Huang, F. J. Mater. Chem. C Mater. Opt. Electron. devices 2014, 2, 6609-6617.

(179) Lee, M.; Lee, S.; Jiang, L. J. AM. CHEM. SOC. 2004, 126, 12724-12725.

(180) Wang, K.; Guo, D.; Zhao, M.; Liu, Y. Chem. Eur. J. 2014, 1475 - 1483. 
(181) Guo, D. S.; Wang, K.; Wang, Y. X.; Liu, Y. J. Am. Chem. Soc. 2012, 134 (24), 10244-10250.

(182) Namgung, R.; Lee, Y. M.; Kim, J.; Jang, Y.; Lee, B.; Kim, I.; Sokkar, P.; Rhee, Y. M.; Hoffman, A. S.; Kim, W. J. Nat. Commun. 2014, 5, 1-12.

(183) Panyam, J.; Labhasetwar, V. Adv. Drug Deliv. Rev. 2003, 55 (3), 329-347.

(184) Zhao, X.; Li, H.; Lee, R. J. Expert Opin. Drug Deliv. 2008, 5 (3), 309-319.

(185) Panyam, J.; Labhasetwar, V. Adv. Drug Deliv. Rev. 2012, 64, 61-71.

(186) Soo, J.; Xu, Q.; Kim, N.; Hanes, J.; Ensign, L. M. Adv. Drug Deliv. Rev. 2016, 99, $28-51$.

(187) Wen, Y.; Zhang, Z.; Li, J. Adv. Funct. Mater. 2014, 3874-3884.

(188) Davis, M. E.; Zuckerman, J. E.; Choi, C. H. J.; Seligson, D.; Tolcher, A.; Alabi, C. A.; Yen, Y.; Heidel, J. D.; Ribas, A. Nature 2010, 464 (7291), 1067-1070.

(189) Merkel, O. M.; Kissel, T. Acc. Chem. Res. 2012, 45, 961-970.

(190) Wang, Y.; Huang, L. Nat. Publ. Gr. 2013, 31 (7), 611-612.

(191) Ramakrishna, V.; Treml, J. F.; Vitale, L.; John, E.; Neill, T. O.; Smith, P. A.; Charles, L.; He, L.; Goldstein, J.; Wallace, P. K.; Endres, M. J.; Ramakrishna, V.; Treml, J. F.; Vitale, L.; Connolly, J. E.; Neill, T. O.; Smith, P. A.; Jones, C. L.; He, L.; Goldstein, J.; Wallace, P. K.; Keler, T.; Endres, M. J. J. Immunol. 2014, 2846-2852.

(192) Dangaj, D.; Abbott, K. L.; Mookerjee, A.; Zhao, A.; Kirby, P. S.; Siegel, D. L.; Wolf, C.; Jr, D. J. P.; Lamazie, A. PLoS One 2011, 6, 28386.

(193) Karthik, S.; Puvvada, N.; Kumar, B. N. P.; Rajput, S.; Pathak, A.; Mandal, M.; Singh, N. D. P. ACS Appl. Mater. Interfaces 2013, 5, 5235-5238.

(194) Pedersen, P. J.; Christensen, M. S.; Ruysschaert, T.; Linderoth, L.; Andresen, T. L.; Melander, F.; Mouritsen, O. G.; Madsen, R.; Clausen, M. H. J. Med. Chem. 2009, 3408-3415.

(195) Sienkiewicz, P.; Bielawski, K.; Bielawska, A.; Pa, J. Eur. J. Pharmacol. 2004, 492, 95-101.

(196) Fonseca, S. B.; Pereira, M. P.; Mourtada, R.; Gronda, M.; Horton, K. L.; Hurren, R.; Minden, M. D.; Schimmer, A. D.; Kelley, S. O. Chem. Biol. 2011, 18 (4), 445-453.

(197) Dias, D. J. S.; Joanitti, G. A.; Azevedo, R. B.; Silva, L. P.; Lunardi, C. N.; Gomes, A. J. J. Biophys. Chem. 2015, 1-13.

(198) Al, J. E. T. ACS Nano 2014, 6, 5939-5952.

(199) Dong, M.; Wang, Y.; Peng, Y. Org. Lett. 2010, 22, 5310-5313.

(200) Li, Y.; Liu, R.; Yang, J.; Ma, G.; Zhang, Z.; Zhang, X. Biomateriais 2014, 35 (36), 9731-9745.

(201) Liu, X.; Zhou, J.; Yu, T.; Chen, C.; Cheng, Q.; Sengupta, K.; Huang, Y.; Li, H.; Liu, C.; Wang, Y.; Posocco, P.; Wang, M.; Cui, Q.; Giorgio, S.; Fermeglia, M.; Qu, F.; Pricl, S.; Shi, Y.; Liang, Z.; Rocchi, P.; Rossi, J. J.; Peng, L. Angew. Chem. Int. Ed. 2014, 53, 11822-11827.

(202) Choi, K. Y.; Silvestre, O. F.; Huang, X.; Hida, N.; Liu, G.; Ho, D. N.; Lee, S.; Lee, S. W.; Hong, J. I.; Chen, X. Nat. Protoc. 2014, 9 (8), 1900-1915.

(203) Liu, X.; Zhou, J.; Yu, T.; Chen, C.; Cheng, Q.; Sengupta, K.; Huang, Y.; Li, H.; Liu, C.; Wang, Y.; Posocco, P.; Wang, M.; Cui, Q.; Giorgio, S.; Fermeglia, M.; Qu, F.; Pricl, S.; Shi, Y.; Liang, Z.; Rocchi, P.; Rossi, J. J.; Peng, L. Angew. Chem. Int. Ed. 2014, 12016-12021.

(204) Takemoto, H.; Miyata, K.; Hattori, S.; Ishii, T.; Suma, T.; Uchida, S.; Nishiyama, N.; Kataoka, K. Angew. Chem. Int. Ed. 2013, 6218-6221.

(205) Varkouhi, A. K.; Scholte, M.; Storm, G.; Haisma, H. J. J. Control. Release 2011, 151 (3), $220-$ 228.

(206) Makki, M. S. I.; Staneva, D.; Sobahi, T. R.; Bosch, P.; Abdel-rahman, R. M.; Grabchev, I. Tetrahedron 2014, 70 (49), 9366-9372. 
(207) Xia, Y.; Peng, L. Chem. Rev. 2012, 113, 7880-7929.

(208) Wei, T.; Chen, C.; Liu, J.; Liu, C.; Posocco, P.; Liu, X.; Cheng, Q. PNAS 2015, 2978-2983.

(209) Ding, D.; Li, K.; Liu, B.; Tang, B. Acc. Chem. Res. 2013, 46, 2441-2453.

(210) Taniguchi, R.; Yamada, T.; Sada, K.; Kokado, K. Macromolecules 2014, 47, 6382-6388.

(211) Li, C.; Wu, T.; Hong, C.; Zhang, G.; Liu, S. Angew. Chem. Int. Ed. 2012, 455-459.

(212) Information, S. Chem. Sci. 2015, 5824-5830.

(213) Li, K.; Zhu, Z.; Cai, P.; Liu, R.; Tomczak, N.; Ding, D.; Liu, J.; Qin, W.; Zhao, Z.; Hu, Y.; Chen, X.; Tang, B. Z.; Liu, B. 2013.

(214) Li, K.; Qin, W.; Ding, D.; Tomczak, N.; Geng, J.; Liu, R.; Liu, J.; Zhang, X.; Liu, H.; Liu, B.; Tang, B. Z. 2012, 1-10.

(215) Arseneault, M.; Liu, B.; Pen, E.; Tang, B. Z. ACS Appi. Mater. Interfaces 2015, 7, 15168-15176.

(216) Ding, D. A. N.; Li, K. A. I.; Liu, B. I. N.; Tang, B. E. N. Z. 2013, 2441-2453.

(217) Li, K.; Zhu, Z.; Cai, P.; Liu, R.; Tomczak, N.; Ding, D.; Liu, J.; Qin, W.; Zhao, Z.; Hu, Y.; Chen, X.; Tang, B. Z.; Liu, B. Chem. Mater 2013, 25, 4181-4187.

(218) Hong, Y.; Lam, W. Y.; Zhong, B. Chem. Soc. Rev 2011, 5361-5388.

(219) Li, K.; Imre, E.; His, A. S.; Liu, B. Chem. Soc. Rev. 2014, 43, 6570-6597.

(220) Zhang, X.; Zhang, X.; Tao, L.; Chi, Z.; Wei, Y. J. Mater. Chem. B 2014, 4398-4414.

(221) Liu, Y.; Tang, Y.; Barashkov, N. N.; Irgibaeva, I. S.; Lam, J. W. Y. J. AM. CHEM. SOC. 2010, 132, 13951-13953.

(222) Noguchi, T.; Roy, B.; Yoshihara, D.; Tsuchiya, Y. Chem. Eur. J. 2014, 381-384.

(223) Sanji, T.; Shiraishi, K.; Nakamura, M.; Tanaka, M. Chem. Asian J 2010, 817-824.

(224) Hu, X.; Chen, Q.; Wang, J.; Cheng, Q.; Yan, C. Chem. Asian J. 2011, 2376-2381.

(225) Huang, G.; Ma, B.; Chen, J.; Peng, Q.; Zhang, G. Chem. Eur. J. 2012, 3886-3892.

(226) Zhang, J.; Li, C.; Wang, Y.; Zhuo, R.; Zhang, X. Chem. Commun 2011, 4457-4459. 


\section{List of the publications}

1. Hongzhong Chen, Huijun Phoebe Tham, Chung Yen Ang, Qiuyu Qu, Lingzhi Zhao, Pengyao Xing, Linyi Bai, Si Yu Tan, Yanli Zhao, ${ }^{*}$ Responsive prodrug self-assembled vesicles for targeted chemotherapy in combination with intracellular imaging. ACS Appl. Mater. Interfaces 2016, 8, 24319-24324.

2. Huijun Phoebe Tham, Hongzhong Chen, Yu Hui Tan, Qiuyu Qu, Sivaramapanicker Sreejith, Lingzhi Zhao, Subbu S. Venkatraman,* Yanli Zhao, ${ }^{*}$ Photosensitizer anchored gold nanorods for targeted combinational photothermal and photodynamic therapy. Chem. Commun. 2016, 52, 8854-8857.

3. JinGui Wang, ${ }^{*}$ Yabo Wang, Hongzhong Chen, Jiesheng Lim, Takashi Tatsumi, Yanli Zhao, ${ }^{*}$ Cation-exchange resin towards low-cost synthesizing high-performance TS-1 zeolites in the presence of alkali-metal ions. RSC Adv. 2016, 6, 15615-15621.

4. Pengyao Xing, Hongzhong Chen, Linyi Bai, Aiyou Hao, ${ }^{*}$ Yanli Zhao,* Superstructure formation and topological evolution achieved by self-organization of a highly adaptive dynamer. ACS Nano 2016, 10, 2716-2727.

5. Pengyao Xing, Hongzhong Chen, Mingfang Ma, Xingdong Xu, Aiyou Hao, Yanli Zhao, Light and cucurbit[7] uril complexation dual-responsiveness of a cyanostilbene-based self-assembled system. Nanoscale 2016, 8, 1892-1896.

6. Xing-Dong Xu, Xin Li, Hongzhong Chen, Qiuyu Qu, Lingzhi Zhao, Hans Ågren, Yanli Zhao, ${ }^{*}$ Host-guest interaction mediated construction of hydrogels and nanovesicles for drug delivery. Small 2015, 11, 5901-5906.

7. Pengyao Xing, Hongzhong Chen, Linyin Bai, Yanli Zhao, ${ }^{*}$ Photo-triggered transformation from vesicles to branched nanotubes fabricated by a cholesterol-appended cyanostilbene. Chem. Commun. 2015, 51, 9309-9312. 